CRYSTALLOGRAPHIC COMMUNICATIONS

ISSN 2056-9890

Received 31 December 2018

Accepted 25 January 2019

Edited by J. Ellena, Universidade de Sâo Paulo, Brazil

Keywords: crystal structure; tetraphosphonate cavitands; inclusion compounds; mephedrone; illicit drugs.

CCDC reference: 1893628

Supporting information: this article has supporting information at journals.iucr.org/e

\section{Crystal structure of a host-guest complex between mephedrone hydrochloride and a tetraphosphonate cavitand}

\author{
Elisa Biavardi and Chiara Massera*
}

Dipartimento di Scienze Chimiche, della Vita e della Sostenibilità Ambientale, Università di Parma, Parco Area delle Scienze 17/A, 43124 Parma, Italy. *Correspondence e-mail: chiara.massera@unipr.it

A new supramolecular complex (I) between the tetraphosphonate cavitand Tiiii $\left[\mathrm{C}_{3} \mathrm{H}_{7}, \mathrm{CH}_{3}, \mathrm{C}_{6} \mathrm{H}_{5}\right.$ ] [systematic name: 2,8,14,20-tetrapropyl-5,11,17,23-tetramethyl-6,10:12,16:18,22:24,4-tetrakis(phenylphosphonato- $O, O^{\prime}$ )resorcin[4]arene] and mephedrone hydrochoride $\left\{\mathrm{C}_{11} \mathrm{H}_{16} \mathrm{NO}^{+} \cdot \mathrm{Cl}^{-}\right.$; systematic name: methyl[1-(4-methylphenyl)-1-oxopropan-2-yl]azanium chloride\} has been obtained and characterized both in solution and in the solid state. The complex of general formula $\left(\mathrm{C}_{11} \mathrm{H}_{16} \mathrm{NO}\right) @$ Tiiii $\left[\mathrm{C}_{3} \mathrm{H}_{7}, \mathrm{CH}_{3}, \mathrm{C}_{6} \mathrm{H}_{5}\right] \mathrm{Cl} \cdot \mathrm{CH}_{3} \mathrm{OH}$ or $\mathrm{C}_{11} \mathrm{H}_{16} \mathrm{NO}^{+} \cdot \mathrm{Cl}^{-} \cdot \mathrm{C}_{68} \mathrm{H}_{68} \mathrm{O}_{12} \mathrm{P}_{4} \cdot \mathrm{CH}_{3} \mathrm{OH}$, crystallizes in the monoclinic space group $P 2_{1} / c$ with one lattice methanol molecule per cavitand, disordered over two positions with occupancy factors of 0.665 (6) and 0.335 (6). The mephedrone guest interacts with the $\mathrm{P}=\mathrm{O}$ groups at the upper rim of the cavitand through two charge-assisted $\mathrm{N}-\mathrm{H}$.. O hydrogen bonds, while the methyl group directly bound to the amino moiety is stabilized inside the $\pi$ basic cavity via cation $\cdots \pi$ interactions. The chloride counter-anion is located between the alkyl legs of the cavitand, forming $\mathrm{C}-\mathrm{H} \cdots \mathrm{Cl}$ interactions with the aromatic and methylenic $\mathrm{H}$ atoms of the lower rim. The chloride anion is also responsible for the formation of a supramolecular chain along the $b$-axis direction through $\mathrm{C}-\mathrm{H} \cdots \mathrm{Cl}$ interactions involving the phenyl substituent of one phosphonate group. $\mathrm{C}-$ $\mathrm{H} \cdots \mathrm{O}$ and $\mathrm{C}-\mathrm{H} \cdots \pi$ interactions between the guest and adjacent cavitands contribute to the formation of the crystal structure.

\section{Chemical context}

Mephedrone (2-methylamino-1- $p$-tolylpropan-1-one), often abbreviated as 4-MMC, the acronym of 4-methyl methcathinone, is a synthetic drug belonging to the family of methamphetamines known for its stimulant effects (Winstock et al., 2010; Morris, 2010; Wood et al., 2010). It can be considered a 'designer drug', that is, a compound resulting from the chemical modification of an existing drug, which in this case is cathinone, a natural alkaloid found in the plant Catha edulis. As a result of the major impact these substances have on human health and social security, it is extremely important to have sensitive, selective and fast methods to identify them as a class, independently from all the synthetic modifications that can be devised to market them and to bypass the legal restrictions to which the parent compounds are subjected. Among the existing analytical methods used to detect 4-MMC in human biological samples or in different media (water, mixtures of powders, etc), solid-phase extraction (SPE) and liquid chromatography combined with mass spectrometry (LC/MS) are the most common, as can be seen from the 
extended literature which has been published on the subject in the past few years (Kolmonen et al., 2009; Singh et al., 2010; Santali et al., 2011; Frison et al., 2011; Strano-Rossi et al., 2012; Power et al., 2012; Perera et al., 2012; Lua et al., 2012; Vircks \& Mulligan, 2012; Concheiro et al., 2013; Mayer et al., 2013; Mwenesongole et al., 2013; Pedersen et al., 2013; Kanu et al., 2013; Strano-Rossi et al., 2014; de Castro et al., 2014; Mercolini et al., 2016; Salomone et al., 2016; Fontanals et al., 2017; Lendoiro et al., 2017; Mercieca et al., 2018; Robin et al., 2018). Recently, the group of Professor Dalcanale has reported a new method to detect methamphetamine salts with extremely high selectivity in water, using cavitand-grafted silicon microcantilevers (Biavardi et al., 2014); more precisely, MDMA (methylenedioxymethamphetamine), cocaine, amphetamine, and 3-fluoromethamphetamine hydrochlorides have been successfully detected in this way. This method takes advantage of the ability shown by tetraphosphonate cavitands to selectively recognize the ${ }^{+} \mathrm{NH}_{2}-\mathrm{CH}_{3}$ group $\left({ }^{+} \mathrm{NHR}-\mathrm{CH}_{3}\right.$ in the case of cocaine) common to all the above-mentioned drug salts through the concomitant formation of $\mathrm{CH}_{3} \cdots \pi$ interactions and hydrogen bonding. Indeed, resorcinarene-based cavitands (Cram, 1983; Cram \& Cram, 1994) decorated at the upper rim with phosphonate groups or quinoxaline moieties have long been exploited for their molecular recognition properties towards charged and neutral molecules (Dutasta, 2004; Vachon et al., 2011; Melegari et al., 2013; Pinalli et al., 2016; Tudisco et al., 2016; Trzciński et al., 2017; Pinalli et al., 2018; Wu et al., 2012; Clément et al., 2015). In order to further assess the recognition properties of tetraphosphonate cavitands towards quaternary ammonium salts of social interest, the supramolecular complex between Tiiii $\left[\mathrm{C}_{3} \mathrm{H}_{7}, \mathrm{CH}_{3}, \mathrm{C}_{6} \mathrm{H}_{5}\right]$ and mephedrone hydrochloride is herein reported and analysed, both in the solid state through the detailed analysis of its crystal and molecular structure, and in solution via NMR studies.
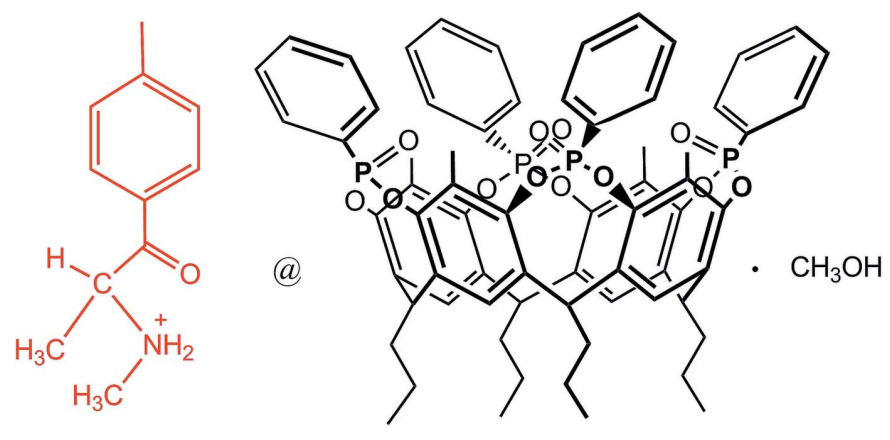

$\mathrm{Cl}^{-}$

\section{Structural commentary}

The host-guest complex (I) of general formula $\left(\mathrm{C}_{11} \mathrm{H}_{16} \mathrm{NO}\right) @$ Tiiii $\left[\mathrm{C}_{3} \mathrm{H}_{7}, \mathrm{CH}_{3}, \mathrm{C}_{6} \mathrm{H}_{5}\right] \mathrm{Cl} \cdot \mathrm{CH}_{3} \mathrm{OH}$ crystallizes in the monoclinic space group $P 21 / c$; its molecular structure is shown in Fig. 1. It consists of a 1:1 inclusion compound between mephedrone hydrochloride and a resorcinarenebased tetraphosphonate cavitand with the four $\mathrm{P}=\mathrm{O}$ groups bridging the upper rim all pointing inwards the aromatic cavity. At the lower rim, four propyl chains are present, one of which is disordered over two equivalent positions with occupancy factors of 0.5 . For each supramolecular complex, one lattice methanol molecule is present, disordered over two positions with occupancy factors of 0.665 (6) and 0.335 (6) (see Fig. 3). The mephedrone cation $\left(\mathrm{C}_{11} \mathrm{H}_{16} \mathrm{NO}\right)^{+}$, which is protonated at the nitrogen atom $\mathrm{N} 1$, is located inside the cavity through the formation of two strong, charge-assisted $\mathrm{N}-\mathrm{H} \cdots \mathrm{O}$ hydrogen bonds involving the $\mathrm{P}=\mathrm{O}$ groups at the

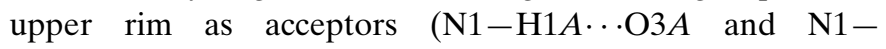
$\mathrm{H} 1 B \cdots \mathrm{O} 3 B$, see Fig. 2 and Table 1 for the detailed geometrical parameters). The methyl group $\mathrm{C} 1$ directly bonded to the amino moiety is located inside the $\pi$ basic cavity, stabilized via a cation $\cdots \pi$ interaction involving the $\mathrm{C} 1-\mathrm{H} 1 D$ moiety and the aromatic ring $\mathrm{C} 1 B-\mathrm{C} 6 B \quad[\mathrm{C} 1-\mathrm{H} 1 D \cdots C g 1$, 3.672 (7) $\AA$ and $145.1^{\circ}$, where $\mathrm{Cg} 1$ is the centroid of the benzene ring]. According to the electrostatic model, the term 'cation $\cdots \pi$ ' is more appropriate than ' $\mathrm{C}-\mathrm{H} \cdots \pi$ ' to describe the interactions of $\mathrm{N}$-methylammonium ions (Dougherty, 2013).] Further stabilization is provided by three C$\mathrm{H}_{\text {guest }} \cdots \mathrm{O}=\mathrm{P}_{\text {host }}$ hydrogen bonds (Fig. 2 and Table 1). The distance of $\mathrm{C} 1$ from the mean plane passing through the methylene atoms $\mathrm{C} 8 A, \mathrm{C} 8 B, \mathrm{C} 8 \mathrm{C}$ and $\mathrm{C} 8 D$ of the lower rim is 3.001 (5) $\AA$, which gives a measure of how deeply the guest is inserted inside the cavity (see also the discussion in Section 5).

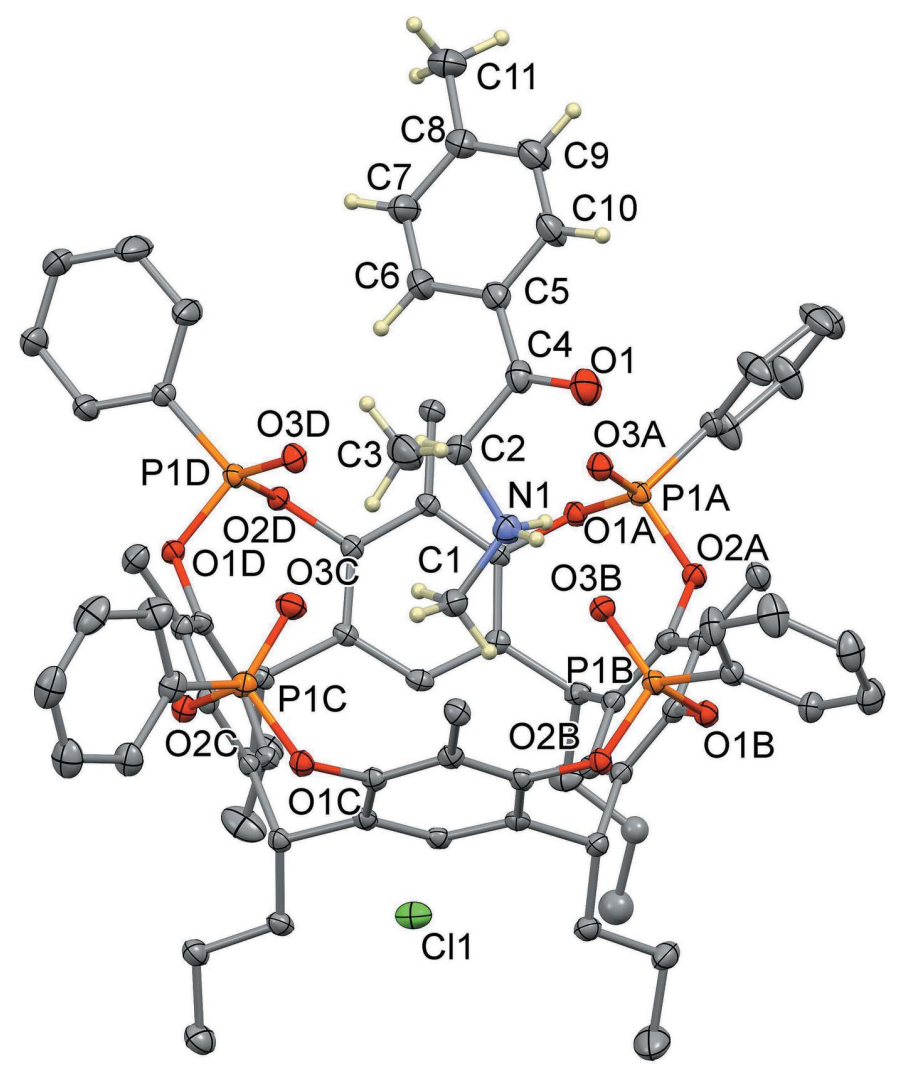

Figure 1

ORTEP view of $\left(\mathrm{C}_{11} \mathrm{H}_{16} \mathrm{NO}\right) @$ Tiiii $\left[\mathrm{C}_{3} \mathrm{H}_{7}, \mathrm{CH}_{3}, \mathrm{C}_{6} \mathrm{H}_{5}\right] \mathrm{Cl}$ (I) with partial atom-labelling scheme and anisotropic displacement parameters drawn at the $20 \%$ probability level. The solvent molecules and the $\mathrm{H}$ atoms of the cavitand are omitted for clarity; only one orientation of the disordered alkyl chain is shown. 
Table 1

Hydrogen-bond geometry $\left(\AA,^{\circ}\right)$.

$\mathrm{Cg} 1$ and $\mathrm{Cg} 2$ are the centroids of the rings $\mathrm{C} 1 B-\mathrm{C} 6 B$ and $\mathrm{C} 1 D-\mathrm{C} 6 D$, respectively.

\begin{tabular}{lllll}
\hline$D-\mathrm{H} \cdots A$ & $D-\mathrm{H}$ & $\mathrm{H} \cdots A$ & $D \cdots A$ & $D-\mathrm{H} \cdots A$ \\
\hline $\mathrm{N} 1-\mathrm{H} 1 A \cdots \mathrm{O} 3 A$ & 0.91 & 1.91 & $2.773(5)$ & 157 \\
$\mathrm{~N} 1-\mathrm{H} 1 B \cdots \mathrm{O} 3 B$ & 0.91 & 1.99 & $2.841(5)$ & 155 \\
$\mathrm{C} 2-\mathrm{H} 2 \cdots \mathrm{O} 3 D$ & 1.00 & 2.25 & $3.140(6)$ & 148 \\
$\mathrm{C} 3-\mathrm{H} 3 A \cdots \mathrm{O} 3 C$ & 0.98 & 2.49 & $3.351(7)$ & 147 \\
$\mathrm{C} 3-\mathrm{H} 3 B \cdots \mathrm{O} 1 S$ & 0.98 & 2.55 & $3.51(2)$ & 164 \\
$\mathrm{O} 2 S-\mathrm{H} 25 \cdots \mathrm{Cl} 1$ & 0.84 & 2.28 & $3.105(5)$ & 169 \\
$\mathrm{C} 1 A-\mathrm{H} 1 A 1 \cdots \mathrm{Cl} 1$ & 0.95 & 2.91 & $3.847(4)$ & 170 \\
$\mathrm{C} 1 B-\mathrm{H} 1 B 1 \cdots \mathrm{Cl} 1$ & 0.95 & 2.93 & $3.870(5)$ & 170 \\
$\mathrm{C} 1 C-\mathrm{H} 1 C 1 \cdots \mathrm{Cl} 1$ & 0.95 & 2.95 & $3.888(5)$ & 170 \\
$\mathrm{C} 1 D-\mathrm{H} 1 D 1 \cdots \mathrm{Cl} 1$ & 0.95 & 2.85 & $3.782(5)$ & 168 \\
$\mathrm{C} 9 A-\mathrm{H} 9 A 1 \cdots \mathrm{Cl} 1$ & 0.99 & 2.76 & $3.738(5)$ & 172 \\
$\mathrm{C} 9 B-\mathrm{H} 9 B 2 \cdots \mathrm{Cl} 1$ & 0.99 & 2.88 & $3.870(4)$ & 175 \\
$\mathrm{C} 9 C-\mathrm{H} 9 C 1 \cdots \mathrm{Cl} 1$ & 0.99 & 2.71 & $3.701(5)$ & 175 \\
$\mathrm{C} 9 D-\mathrm{H} 9 D 1 \cdots \mathrm{Cl} 1$ & 0.99 & 2.85 & $3.838(5)$ & 178 \\
$\mathrm{C} 1-\mathrm{H} 1 D \cdots \mathrm{Cg} 1$ & 0.98 & 2.83 & $3.672(7)$ & 145 \\
$\mathrm{C} 17 D^{\mathrm{i}}-\mathrm{H} 17 D^{\mathrm{i}} \cdots \mathrm{O} 1$ & 0.95 & 2.56 & $3.204(6)$ & 125 \\
$\mathrm{C} 10-\mathrm{H} 10 \cdots \mathrm{O} 1 D^{\mathrm{i}}$ & 0.95 & 2.69 & $3.555(4)$ & 152 \\
$\mathrm{C} 9-\mathrm{H} 9 \cdots \mathrm{Cg} 2^{\mathrm{i}}$ & 0.95 & 2.69 & $3.594(5)$ & 159 \\
$\mathrm{C} 14 B-\mathrm{H} 14 B \cdots \mathrm{Cl} 11^{\mathrm{ii}}$ & 0.95 & 2.89 & $3.697(6)$ & 143 \\
\hline
\end{tabular}

Symmetry codes: (i) $-x+1, y+\frac{1}{2},-z+\frac{3}{2}$; (ii) $-x, y+\frac{1}{2},-z+\frac{3}{2}$.

The chloride anion is located between the alkyl legs of the cavitand, with a $\mathrm{Cl} 1 \cdots \mathrm{N} 1$ distance of 7.097 (5) $\AA$, forming numerous $\mathrm{C}-\mathrm{H} \cdots \mathrm{Cl}$ interactions with the aromatic and methylenic hydrogen atoms of the lower rim (see Table 1), as well as a hydrogen bond with the $\mathrm{O} 2 S-\mathrm{H} 2 S$ group of the methanol molecule of occupancy factor 0.665 (6) [O $2 S-$ $\mathrm{H} 2 S$. C Cl1, 3.105 (5) $\AA$ and $168.5^{\circ}$ ]. Moreover, the O1S atom from the other methanol fraction accepts a hydrogen bond from the methyl group $\mathrm{C} 3$ of the mephedrone guest [C3$\mathrm{H} 3 B \cdots \mathrm{O} 1 S, 3.51$ (2) $\AA$ and $164.3^{\circ}$ ].

\section{Supramolecular features}

Besides the supramolecular interactions that yield the 1:1 host-guest complex, mephedrone hydrochloride also influences the overall packing of the crystal structure, as can be

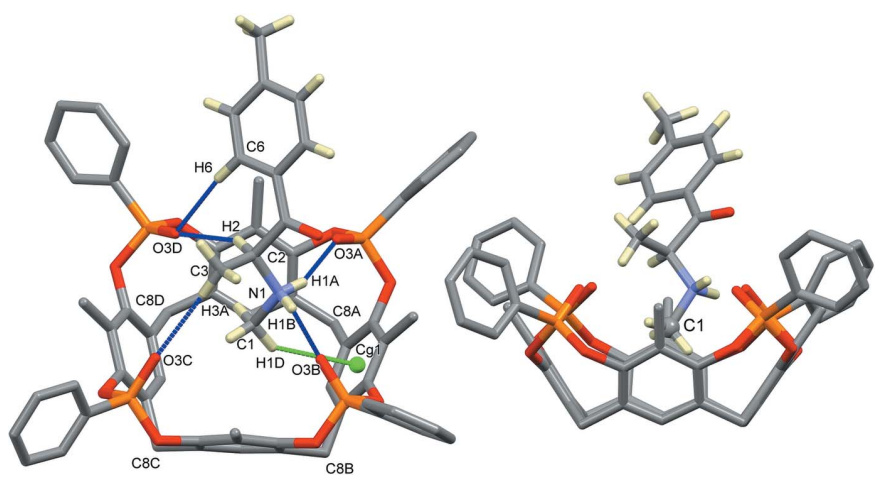

Figure 2

Left: view of the main host-guest supramolecular interactions shown as blue and green dotted lines. Only relevant $\mathrm{H}$ atoms are shown, while the alkyl chains, the chloride anion and the methanol lattice molecules have been omitted for clarity. Right: side view of the host-guest complex.

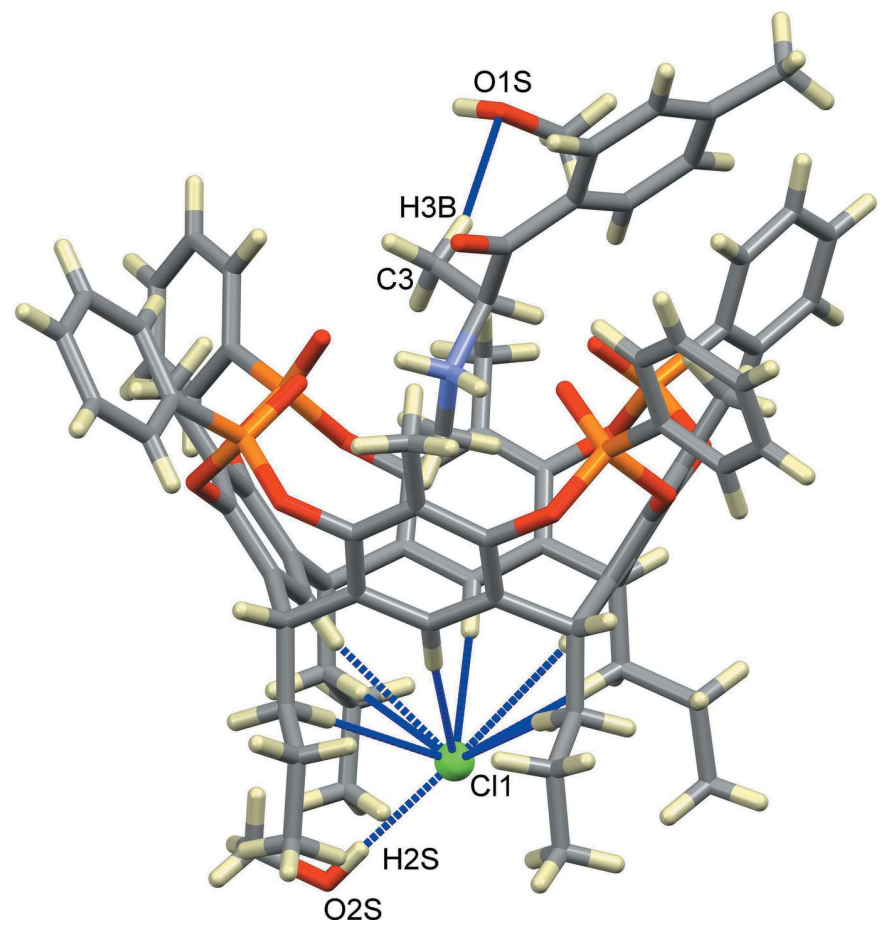

Figure 3

Supramolecular interactions (blue dotted lines) involving the chloride anion (represented as a green sphere) and the disordered methanol lattice molecules.

seen from Figs. 4 and 5. The chloride anion is responsible for the formation of a supramolecular chain along the $b$-axis direction through $\mathrm{C} 14 B-\mathrm{H} 14 B \cdots \mathrm{Cl}^{-}\left(-x, \frac{1}{2}+y, \frac{3}{2}-z\right)$ contacts involving the phenyl substituents of one of the four phosphonate groups (Fig. 4). On the other side, the cationic part of the guest is involved in $\mathrm{C}-\mathrm{H} \cdots \mathrm{O}$ and $\mathrm{C}-\mathrm{H} \cdots \pi$ interactions with the phenyl ring bound to the $\mathrm{P} 1 D=\mathrm{O} 3 D$ group and the aromatic ring $\mathrm{C} 1 D-\mathrm{C} 6 D$ belonging to the wall of an adjacent cavitand (Fig. 5 and Table 2). More precisely, the oxygen atom $\mathrm{O} 1$ of the guest acts as a hydrogen-bond acceptor towards the $\mathrm{C} 17 D^{\mathrm{i}}-\mathrm{H} 17 D^{\mathrm{i}}$ group [3.204 (6) $\AA$ and $125.0^{\circ}$; symmetry code (i): $-x+1, y+\frac{1}{2},-z+\frac{3}{2}$ ], while $\mathrm{C} 9-\mathrm{H} 9$ and $\mathrm{C} 10-\mathrm{H} 10$ act as donors towards the centroid $\mathrm{Cg} 2^{\mathrm{i}}$ $\left[3.594(5) \AA\right.$ and $158.7^{\circ}$ ] and the oxygen atom O1D [3.555 (4) $\AA$ and $151.8^{\circ}$ ], respectively. These sets of interactions can be summarized visually by calculating the twodimensional fingerprint plots derived from the Hirshfeld surface analysis (Spackman \& McKinnon, 2002; McKinnon et al., 2004), using the program Crystal Explorer 17 (Turner et al., 2017). The overall fingerprint plot for (I) is shown in Fig. $6 a$ and those delineated in $\mathrm{H} \cdots \mathrm{H}(67.8 \%), \mathrm{C} \cdot \mathrm{H} / \mathrm{H} \cdots \mathrm{C}$ $(23.3 \%), \mathrm{O} \cdots \mathrm{H} / \mathrm{H} \cdots \mathrm{O}(6.4 \%)$ and $\mathrm{Cl} \cdots \mathrm{H} / \mathrm{H} \cdots \mathrm{Cl}(1.2 \%)$ interactions are shown in Fig. $6 b-e$, respectively (the methanol solvent and the disordered alkyl chain have been omitted from the calculation). Apart from the $\mathrm{H} \cdots \mathrm{H}$ contacts, which probably derive from the interactions involving the alkyl chains, the second highest contribution arises from $\mathrm{C} \cdots \mathrm{H} /$ $\mathrm{H} \cdots \mathrm{C}$ contacts $\left(d_{\mathrm{i}}+d_{\mathrm{e}} \sim 2.58 \AA\right)$, followed by $\mathrm{O} \cdots \mathrm{H} / \mathrm{H} \cdots \mathrm{O}$ $\left(d_{\mathrm{i}}+d_{\mathrm{e}} \sim 2.48 \AA\right)$ and $\mathrm{Cl} \cdots \mathrm{H} / \mathrm{H} \cdots \mathrm{Cl}\left(d_{\mathrm{i}}+d_{\mathrm{e}} \sim 2.76 \AA\right)$, all shorter than the respective sums of the van der Waals radii. 


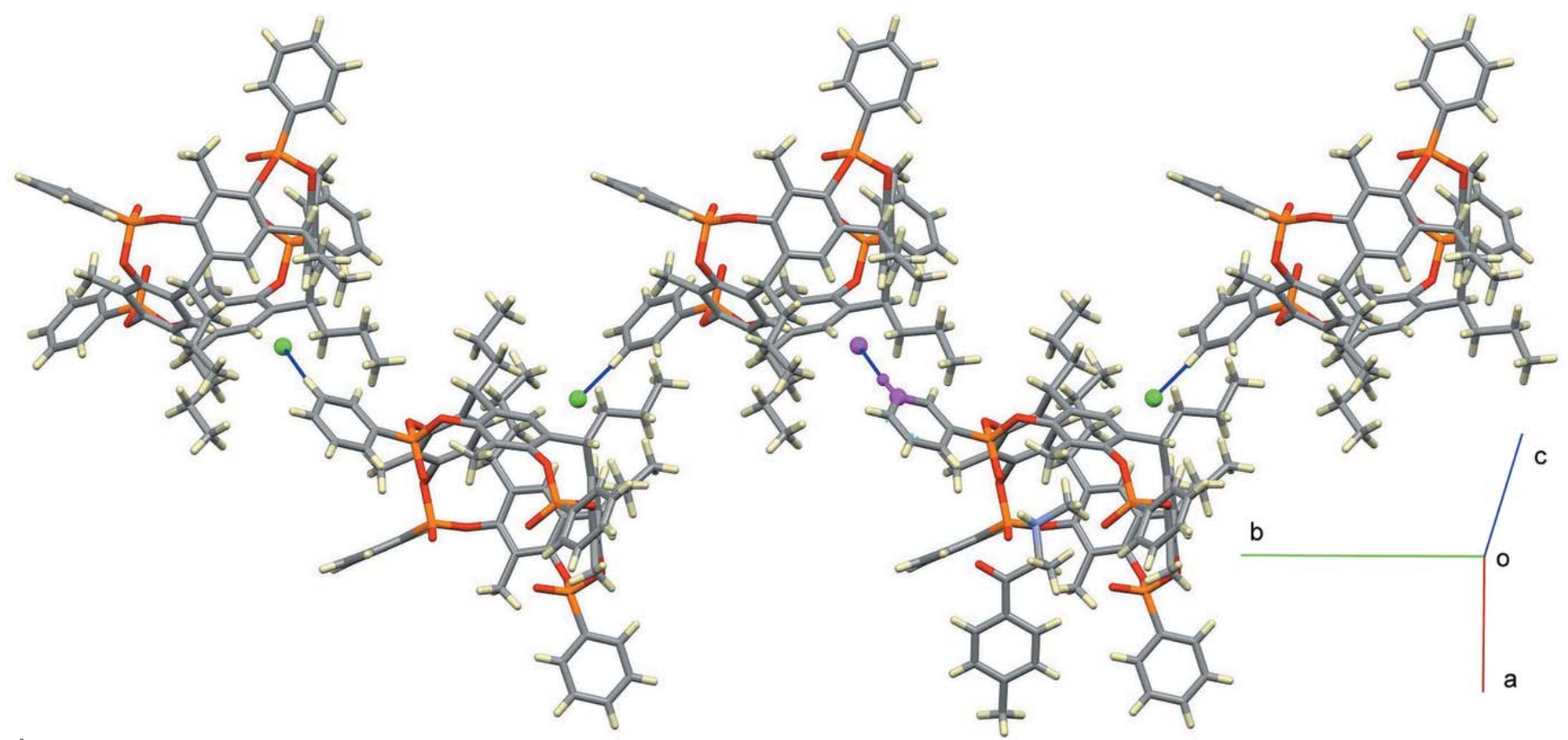

Figure 4

View of the packing of (I) along the $b$-axis direction, mediated by $\mathrm{C} 14 B-\mathrm{H} 14 B \cdots \mathrm{Cl}^{-}$interactions (blue lines). The $\mathrm{C}$ and $\mathrm{H}$ atoms highlighted in purple are in general positions, while the chloride anion is at the symmetry position $-x, \frac{1}{2}+y, \frac{3}{2}-z$.

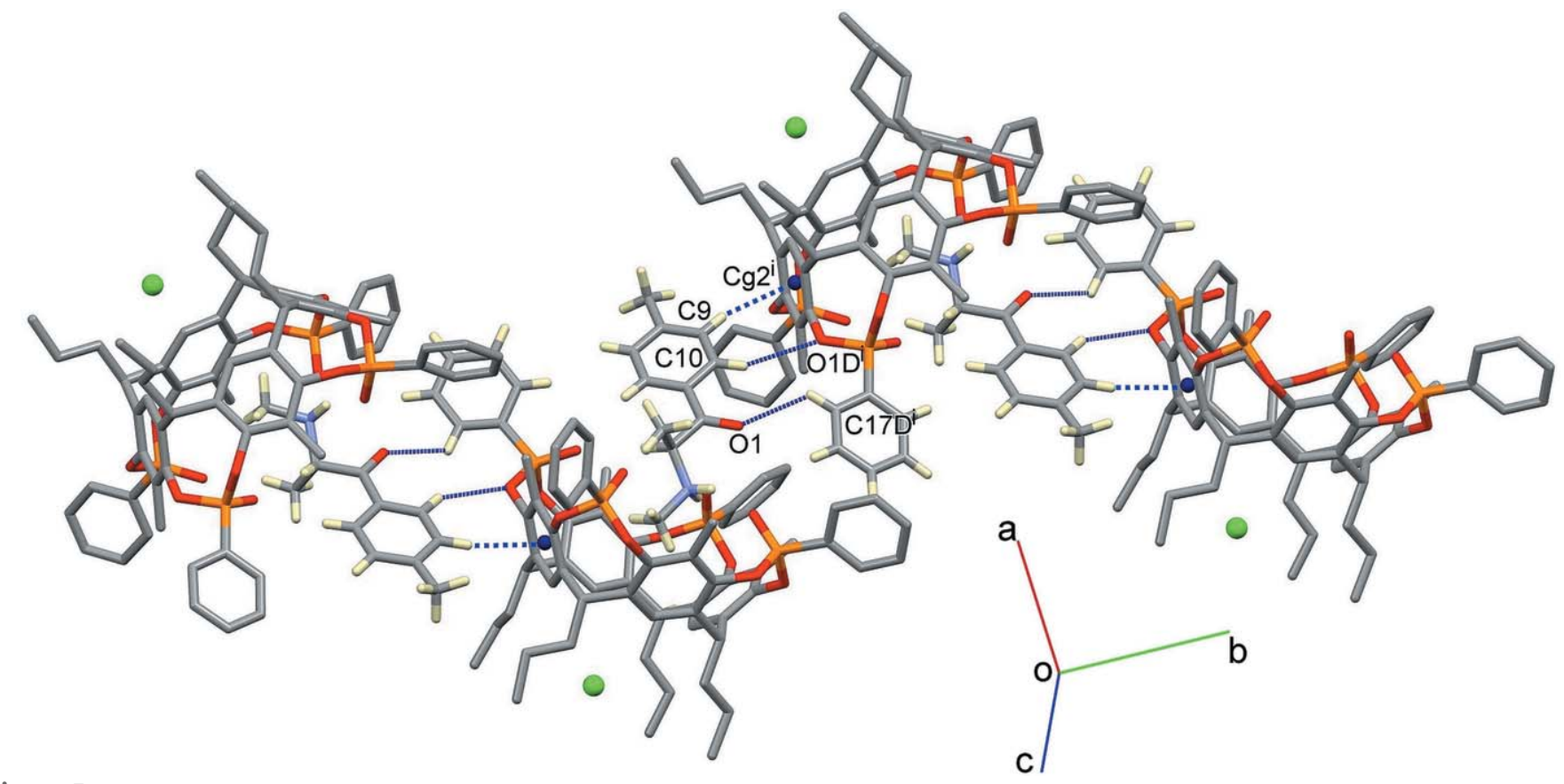

Figure 5

View of the packing of (I) mediated by $\mathrm{C}-\mathrm{H} \cdots \mathrm{O}$ and $\mathrm{C}-\mathrm{H} \cdots \pi$ interactions between the guests and adjacent cavitands. Symmetry code: (i) $1-x, \frac{1}{2}+y$, $\frac{3}{2}-z$.

\section{Studies in solution}

In solution, complexation was observed both via phosphorous and proton NMR spectroscopy following the shift of the ${ }^{31} \mathrm{P}$ signals of the Tiiii $\left[\mathrm{C}_{3} \mathrm{H}_{7}, \mathrm{CH}_{3}, \mathrm{Ph}\right]$ host and the shift of the ${ }^{+} \mathrm{N}-\mathrm{CH}_{3}$ protons of the mephedrone hydrochloride guest. The titration was performed in deuterated methanol at $253 \mathrm{~K}$, in order to be under slow chemical exchange in the NMR time scale and better observe the complexation event. The NMR tube was filled with $0.4 \mathrm{~mL}$ of a deuterated methanol solution containing the cavitand $(7.5 \mathrm{mM}$ concentration). The mephedrone hydrochloride titrant solution was prepared by dissolving the guest in $0.1 \mathrm{~mL}$ of deuterated methanol $(31 \mathrm{mM})$. Two portions ( 0.5 eq., $48.5 \mathrm{~mL}$ ) of the titrant were added by syringe to the NMR tube. During the titration, the phosphorous singlet of the cavitand shifted downfield, from 8.70 (free host) to $11.14 \mathrm{ppm}$ upon addition of one equivalent of the guest (Fig. $7 a$ and $7 c$ ), indicating the presence of cation- 
(a)

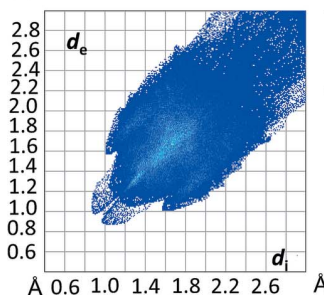

(b)

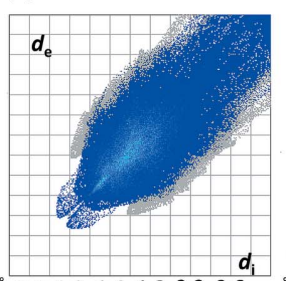

(c)

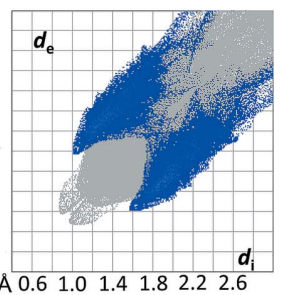

(d)

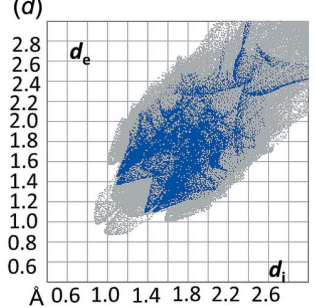

(e)

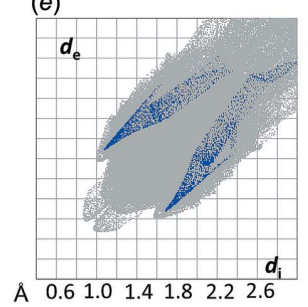

Figure 6

The full two-dimensional fingerprint plot $(a)$ and those delineated into $\mathrm{H} \cdots \mathrm{H}(b), \mathrm{C} \cdots \mathrm{H} / \mathrm{H} \cdots \mathrm{C}(c), \mathrm{O} \cdots \mathrm{H} / \mathrm{H} \cdots \mathrm{O}(d)$ and $\mathrm{Cl} \cdots \mathrm{H} / \mathrm{H} \cdots \mathrm{Cl}(e)$ contacts for (I).

dipole interactions between the ${ }^{+} \mathrm{N}-\mathrm{CH}_{3}$ and the phosphonate groups at the upper rim. The addition of 0.5 eq. of guest caused the appearance of two phosphorous signals at 8.74 and $11.14 \mathrm{ppm}$ related to the free host and to the complex, respectively (Fig. $7 b$ ).

In the proton NMR, after the addition of 0.5 equivalent of mephedrone hydrochloride the diagnostic upfield shift of the

(c)

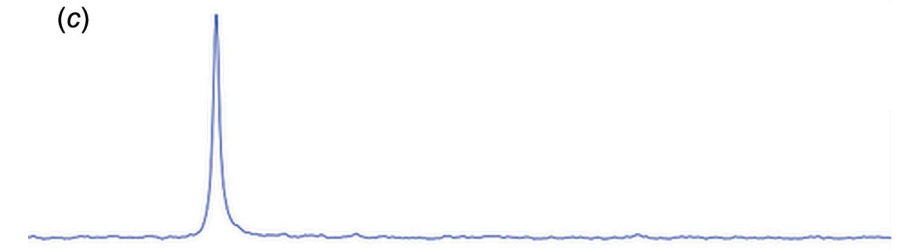

(b)

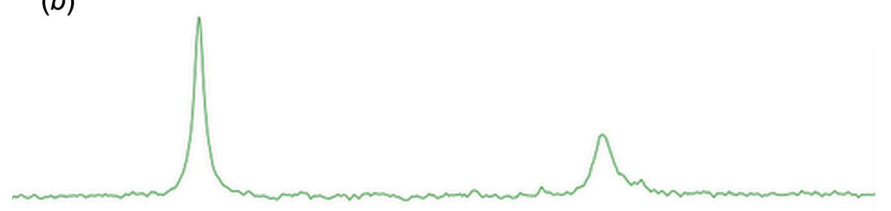

(a)

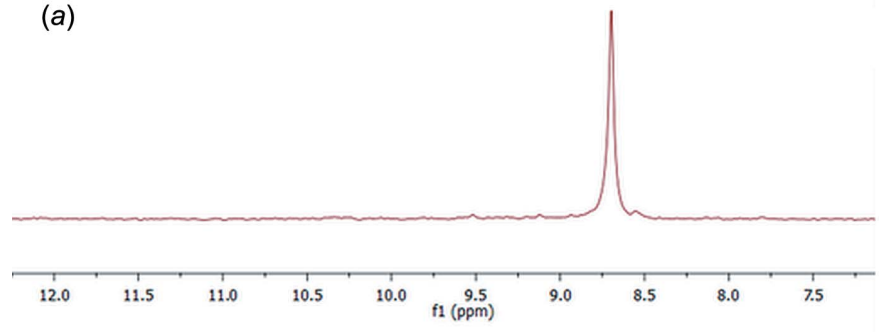

Figure 7

${ }^{31} \mathrm{P}$ NMR (162 MHz, MeOD, $253 \mathrm{~K}$ ) spectra of (a) free host Tiiii $\left[\mathrm{C}_{3} \mathrm{H}_{7}\right.$, $\mathrm{CH}_{3}, \mathrm{Ph}$; (b) addition of 0.5 equivalent of mephedrone $\mathrm{HCl}$ to the host solution; (c) addition of 1 equivalent of mephedrone $\mathrm{HCl}$ to the host solution.

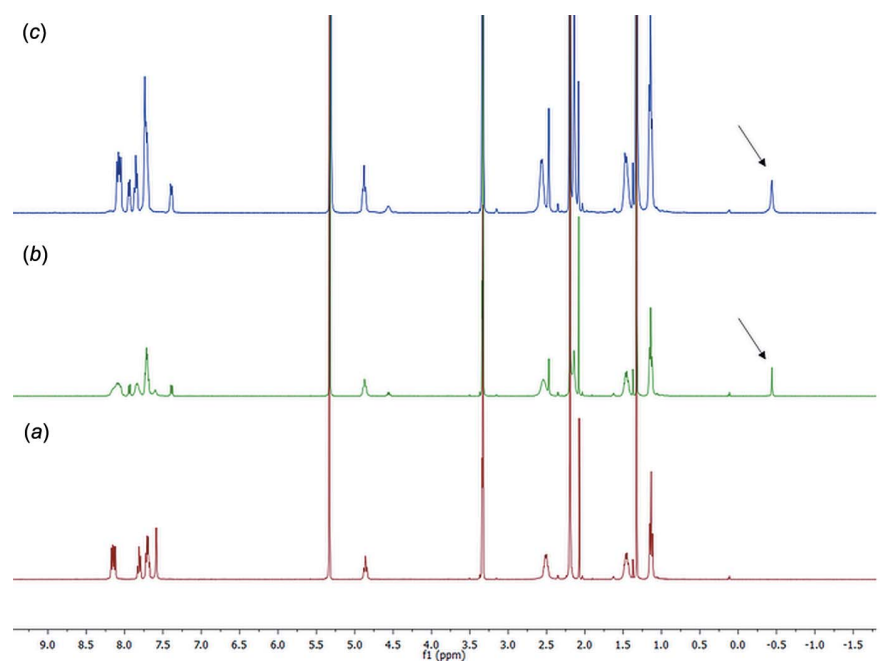

Figure 8

${ }^{1} \mathrm{H}$ NMR (400 MHz, MeOD, $253 \mathrm{~K}$ ) spectra of (a) free host Tiiii $\left[\mathrm{C}_{3} \mathrm{H}_{7}\right.$, $\left.\mathrm{CH}_{3}, \mathrm{Ph}\right] ;(b)$ addition of 0.5 equivalent of mephedrone $\mathrm{HCl}$ to the host solution; (c) addition of 1 equivalent of mephedrone $\mathrm{HCl}$ to the host solution. The arrows indicate the up-shift of ${ }^{+} \mathrm{N}-\mathrm{CH}_{3}$ protons.

guest ${ }^{+} \mathrm{N}-\mathrm{CH}_{3}$ signals was observed, as expected for the shielding effect caused by its inclusion in the aromatic cavity of the host (Fig. 8b). After the addition of one equivalent of guest, the ${ }^{+} \mathrm{N}-\mathrm{CH}_{3}$ singlet appeared still shifted upfield but broadened (Fig. 8c).

\section{Database survey}

As already discussed in Section 1, tetraphosphonate cavitands of general formula Tiiii $[R, R 1, R 2]$ (where $R, R 1$ and $R 2$ are the substituents at the lower rim, on the four benzene rings of the cavity, and on the phosphonate groups, respectively; Pinalli et al., 2004), are excellent receptors for molecular recognition of neutral and charged guests because of the presence of $\mathrm{P}=\mathrm{O}$ groups that act as hydrogen-bond acceptors, and of the aromatic cavity that allows the formation of $\mathrm{C}-$ $\mathrm{H} \cdots \pi$ interactions. The substituent $R$ at the lower rim can be modified to tune the solubility of the host, to enhance the crystallization process, or to graft the cavity on different surfaces, but does not play any significant role in the recognition process, if not that of interacting with the anionic counterpart of a positively charged guest. A search in the Cambridge Structural Database (Version 5.38, update August 2018; Groom et al., 2016) for a tetraphosphonate scaffold without limitations on $R, R 1$ and $R 2$ yielded 82 hits, with the most populated class (44 hits) being the one of general formula Tiiii[ $\left.\mathrm{H}, \mathrm{CH}_{3}, \mathrm{CH}_{3}\right]$. The substitution of the alkyl chains with hydrogen atoms favours the formation of crystals, albeit lowering the solubility of the macrocycle, and the methyl group on the phosphonate moiety generates less steric hindrance than a phenyl one. Besides these general considerations, the most interesting structural comparisons with the title compound are to be made with supramolecular complexes in which the guests are: (i) the zwitterionic species 1,1-dicyano-2-(dicyanomethyl)-3-(dicyanomethylene)-4,4-bis- 


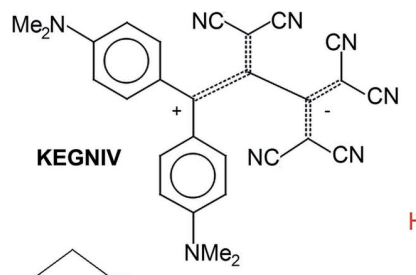<smiles>C[NH2+]C(C)C(O)c1ccccc1</smiles>

MOXREY/MOXRIC
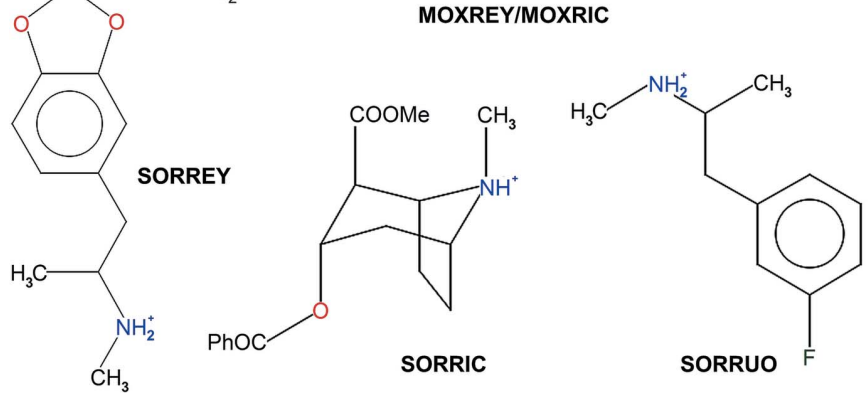

Figure 9

Molecular sketch of the different guests described in the Database survey.

[4-(dimethylamino)phenyl]but-4-ylium-2-ide (KEGNIV; Wu et al., 2012); (ii) the diasteromeric pair ephedrine and pseudoephedrine hydrochloride (MOXREY and MOXRIC; Biavardi et al., 2015); (iii) MDMA, cocaine, amphetamine and 3-fluoromethamphetamine hydrochloride (SORREY, SORRIC, SORROI and SORRUO; Biavardi et al., 2014). A molecular sketch of the guests is reported in Fig. 9. In the case of KEGNIV, the positive charge of the zwitterionic species is localized on the $\mathrm{N}, \mathrm{N}$-dimethylanilino rings, in particular on the $\mathrm{NMe}_{2}$ moiety, and that has been demonstrated by the supramolecular complex formed with Tiiii in which the guest enters the cavity with the positive fragment to form ion-dipole interactions with the $\mathrm{P}=\mathrm{O}$ groups. Ephedrine and pseudoephedrine are complexed by the cavitand via a set of supramolecular contacts very similar to those present in the title compound, that is, hydrogen bonding involving the $-\mathrm{NH}_{2}{ }^{+}$ fragment as donor and the phosphonate groups as acceptors, and cation $\cdots \pi$ interactions. The distance of the carbon atom of the methyl group interacting with the cavity from the mean plane passing through the methylene atoms $\mathrm{C} 8 A, \mathrm{C} 8 B, \mathrm{C} 8 C$ and $C 8 D$ of the lower rim (the labelling is the same as in Fig. 2) is 3.023 (4) $\AA$ for ephedrine, 3.202 (3) $\AA$ for the sterically hindered pseudoephedrine and 3.001 (5) $\AA$ for (I). This value is of 3.122 (2), 4.104 (4), 2.853 (3) and 2.983 (5) $\AA$ for MDMA, cocaine, amphetamine and 3-fluoromethamphetamine hydrochloride, respectively, all in good agreement with that of the title compound (cocaine is less included inside the cavity because of its bulky substituents).

\section{Synthesis and crystallization}

${ }^{1} \mathrm{H}$ NMR spectra were obtained using a Bruker AMX-400 $(400 \mathrm{MHz})$ spectrometer. All chemical shifts $(\delta)$ were reported in ppm relative to the proton resonances resulting from incomplete deuteration of the NMR solvents. ${ }^{31} \mathrm{P}$ NMR spectra were obtained using a Bruker AMX-400 (162 MHz) spectrometer. All chemical shifts $(\delta)$ were recorded in ppm

Table 2

Experimental details.

Crystal data

Chemical formula

$M_{\mathrm{r}}$

Crystal system, space group

Temperature (K)

$a, b, c(\AA)$

$\beta\left(^{\circ}\right)$

$V\left(\AA^{3}\right)$

$Z$

Radiation type

$\mu\left(\mathrm{mm}^{-1}\right)$

Crystal size (mm)

Data collection

Diffractometer

Absorption correction

$T_{\min }, T_{\max }$

No. of measured, independent and observed $[I>2 \sigma(I)]$ reflections

$R_{\text {int }}$

$(\sin \theta / \lambda)_{\max }\left(\AA^{-1}\right)$

Refinement

$R\left[F^{2}>2 \sigma\left(F^{2}\right)\right], w R\left(F^{2}\right), S$

No. of reflections

No. of parameters

No. of restraints

$\mathrm{H}$-atom treatment

$\Delta \rho_{\max }, \Delta \rho_{\min }\left(\mathrm{e} \AA^{-3}\right)$

Computer programs: APEX2 and SAINT (Bruker, 2008), SIR97 (Altomare et al., 1999), SHELXL2014/7 (Sheldrick, 2015), Mercury (Macrae et al., 2006), WinGX (Farrugia, 2012), PARST (Nardelli, 1995) and publCIF (Westrip, 2010).

relative to external $85 \% \mathrm{H}_{3} \mathrm{PO}_{4}$ at $0.00 \mathrm{ppm}$. The cavitand Tiiii $\left[\mathrm{C}_{3} \mathrm{H}_{7}, \mathrm{CH}_{3}, \mathrm{C}_{6} \mathrm{H}_{5}\right]$ was prepared following published procedures (Biavardi et al., 2008). Mephedrone hydrochloride in its racemic form was purchased from SALAR SpA (Italy) and used as received without further purification.

$\left(\mathrm{C}_{11} \mathrm{H}_{16} \mathrm{NO}\right) @$ Tiiii $\left[\mathrm{C}_{3} \mathrm{H}_{7}, \quad \mathrm{CH}_{3}, \quad \mathrm{C}_{6} \mathrm{H}_{5}\right] \mathrm{Cl} \cdot \mathrm{CH}_{3} \mathrm{OH}$ was obtained by mixing a methanol solution of Tiiii $\left[\mathrm{C}_{3} \mathrm{H}_{7}, \mathrm{CH}_{3}\right.$, $\mathrm{C}_{6} \mathrm{H}_{5}$ ] (1 eq.) with a dichloromethane solution of $\mathrm{C}_{11} \mathrm{H}_{16} \mathrm{NOCl}$ (1 eq.). The mixture was left to evaporate to yield colourless single crystals of the 1:1 complex which were suitable for $\mathrm{X}$-ray diffraction analysis.

\section{Refinement}

Crystal data, data collection and structure refinement details are summarized in Table 2. The $\mathrm{H}$ atoms bound to $\mathrm{C}, \mathrm{N}$ and $\mathrm{O}$ were placed in calculated positions and refined isotropically using a riding model with $\mathrm{C}-\mathrm{H}$ ranging from 0.95 to $1.00 \AA$, $\mathrm{N}-\mathrm{H}=0.91 \AA, \mathrm{O}-\mathrm{H}=0.98 \AA$ and $\operatorname{Uiso}(\mathrm{H})$ set to $1.2-$ $1.5 \mathrm{Ueq}(\mathrm{C} / \mathrm{N} / \mathrm{O})$. For each cavitand:guest complex, a methanol solvent molecule was located in the difference-Fourier map, disordered over two positions with occupancy factors of 0.665 (6) and 0.335 (6). One of the four alkyl chains of the cavitand was also found to be disordered over two equivalent positions with occupancy factors of 0.5 , and the relative carbon 
atoms were refined isotropically. Four reflections showing poor agreement $(031, \overline{2} 31,020$ and 231$)$ were omitted from the final refinement.

\section{Acknowledgements}

The Centro Interfacoltà di Misure 'G. Casnati' and the 'Laboratorio di Strutturistica Mario Nardelli' of the University of Parma are kindly acknowledged for the use of NMR and Maldi-MS facilities and of the diffractometer. Permission to use small quantities of illicit drugs has been granted in the framework of the FP7 Dirac project by the Italian Ministero della Salute.

\section{Funding information}

Funding for this research was provided by: European Union through the DIRAC project (award No. FP7-SEC-2009242309); Regione Lombardia-INSTM, SNAF project .

\section{References}

Altomare, A., Burla, M. C., Camalli, M., Cascarano, G. L., Giacovazzo, C., Guagliardi, A., Moliterni, A. G. G., Polidori, G. \& Spagna, R. (1999). J. Appl. Cryst. 32, 115-119.

Biavardi, E., Battistini, G., Montalti, M., Yebeutchou, R. M., Prodi, L. \& Dalcanale, E. (2008). Chem. Commun. pp. 1638.

Biavardi, E., Federici, S., Tudisco, C., Menozzi, D., Massera, C., Sottini, A., Condorelli, G. G., Bergese, P. \& Dalcanale, E. (2014). Angew. Chem. Int. Ed. 53, 9183-9188.

Biavardi, E., Ugozzoli, F. \& Massera, C. (2015). Chem. Commun. 51, 3426-3429.

Bruker (2008). APEX2, SAINT and SADABS. Bruker AXS Inc., Madison, Wisconsin, USA.

Castro, A. de, Lendoiro, E., Fernández-Vega, H., Steinmeyer, S., López-Rivadulla, M. \& Cruz, A. (2014). J. Chromatogr. A, 1374, 93-101.

Clément, P., Korom, S., Struzzi, C., Parra, E. J., Bittencourt, C., Ballester, P. \& Llobet, E. (2015). Adv. Funct. Mater. 25, 4011-4020.

Concheiro, M., Anizan, S., Ellefsen, K. \& Huestis, M. A. (2013). Anal. Bioanal. Chem. 405, 9437-9448.

Cram, D. J. (1983). Science, 219, 1177-1183.

Cram, D. J. \& Cram, J. M. (1994). Container Molecules and their Guests, Monographs in Supramolecular Chemistry, vol. 4, edited by J. F. Stoddart. Royal Society of Chemistry, Cambridge.

Dougherty, D. A. (2013). Acc. Chem. Res. 46, 885-893.

Dutasta, J.-P. (2004). Top. Curr. Chem. 232, 55-91.

Farrugia, L. J. (2012). J. Appl. Cryst. 45, 849-854.

Fontanals, N., Marcé, R. M. \& Borrull, F. (2017). J. Chromatogr. A, 1524, 66-73.

Frison, G., Gregio, M., Zamengo, L., Zancanaro, F., Frasson, S. \& Sciarrone, R. (2011). Rapid Commun. Mass Spectrom. 25, 387-390.

Groom, C. R., Bruno, I. J., Lightfoot, M. P. \& Ward, S. C. (2016). Acta Cryst. B72, 171-179.

Kanu, A. B., Brandt, S. D., Williams, M. D., Zhang, N. \& Hill, H. H. (2013). Anal. Chem. 85, 8535-8542.

Kolmonen, M., Leinonen, A., Kuuranne, T., Pelander, A. \& Ojanperä, I. (2009). Drug Test. Anal. 1, 250-266.

Lendoiro, E., Jiménez-Morigosa, C., Cruz, A., Páramo, M., LópezRivadulla, M. \& de Castro, A. (2017). Drug Test. Anal. 9, 96-105.

Lua, I. A., Lin, S.-L., Lin, H. R. \& Lua, A. C. (2012). J. Anal. Toxicol. 36, 575-581.

Macrae, C. F., Edgington, P. R., McCabe, P., Pidcock, E., Shields, G. P., Taylor, R., Towler, M. \& van de Streek, J. (2006). J. Appl. Cryst. 39, 453-457.
Mayer, M., Benko, A., Huszár, A., Sipos, K., Lajtai, A., Lakatos, A. \& Porpáczy, Z. (2013). J. Chromatogr. Sci. 51, 851-856.

McKinnon, J. J., Spackman, M. A. \& Mitchell, A. S. (2004). Acta Cryst. B60, 627-668.

Melegari, M., Massera, C., Pinalli, R., Yebeutchou, R. M. \& Dalcanale, E. (2013). Sens. Actuators B, 179, 74-80.

Mercieca, G., Odoardi, S., Cassar, M. \& Strano Rossi, S. (2018). J. Pharm. Biomed. Anal. 149, 494-501.

Mercolini, L., Protti, M., Catapano, M. C., Rudge, J. \& Sberna, A. E. (2016). J. Pharm. Biomed. Anal. 123, 186-194.

Morris, K. (2010). Lancet, 375, 1333-1334.

Mwenesongole, E. M., Gautam, L., Hall, S. W., Waterhouse, J. W. \& Cole, M. D. (2013). Anal. Methods 5, 3248-3254.

Nardelli, M. (1995). J. Appl. Cryst. 28, 659.

Pedersen, A. J., Dalsgaard, P. W., Rode, A. J., Rasmussen, B. S., Müller, I. B., Johansen, S. S. \& Linnet, K. (2013). J. Sep. Sci. 36, 2081-2089.

Perera, R. W. H., Abraham, I., Gupta, S., Kowalska, P., Lightsey, D., Marathaki, C., Singh, N. S. \& Lough, W. J. (2012). J. Chromatogr. A, 1269, 189-197.

Pinalli, R., Dalcanale, E., Ugozzoli, F. \& Massera, C. (2016). CrystEngComm, 18, 5788-5802.

Pinalli, R., Pedrini, A. \& Dalcanale, E. (2018). Chem. Eur. J. 24, 10101019.

Pinalli, R., Suman, M. \& Dalcanale, E. (2004). Eur. J. Org. Chem. pp. 451-462.

Power, J. D., McDermott, S. D., Talbot, B., O’Brien, J. E. \& Kavanagh, P. (2012). Rapid Commun. Mass Spectrom. 26, 2601-2611.

Robin, T., Barnes, A., Dulaurent, S., Loftus, N., Baumgarten, S., Moreau, S., Marquet, P., El Balkhi, S. \& Saint-Marcoux, F. (2018). Anal. Bioanal. Chem. 410, 5071-5083.

Salomone, A., Gazzilli, G., Di Corcia, D., Gerace, E. \& Vincenti, M. (2016). Anal. Bioanal. Chem. 408, 2035-2042.

Santali, E. Y., Cadogan, A.-K., Daeid, N. N., Savage, K. A. \& Sutcliffe, O. B. (2011). J. Pharm. Biomed. Anal. 56, 246-255.

Sheldrick, G. M. (2015). Acta Cryst. C71, 3-8.

Singh, N., Day, P., Katta, V. R., Mohammed, G. P. \& Lough, W. J. (2010). J. Pharm. Pharmacol. 62, 1209-1210.

Spackman, M. A. \& McKinnon, J. J. (2002). CrystEngComm, 4, 378392.

Strano-Rossi, S., Anzillotti, L., Castrignanò, E., Romolo, F. S. \& Chiarotti, M. (2012). J. Chromatogr. A, 1258, 37-42.

Strano-Rossi, S., Odoardi, S., Fisichella, M., Anzillotti, L., Gottardo, R. \& Tagliaro, F. (2014). J. Chromatogr. A, 1372, 145-156.

Trzciński, J. W., Pinalli, R., Riboni, N., Pedrini, A., Bianchi, F., Zampolli, S., Elmi, I., Massera, C., Ugozzoli, F. \& Dalcanale, E. (2017). ACS Sens. 2, 590-598.

Tudisco, C., Fragalà, M. E., Giuffrida, A. E., Bertani, F., Pinalli, R., Dalcanale, E., Compagnini, G. \& Condorelli, G. G. (2016). J. Phys. Chem. C, 120, 12611-12617.

Turner, M. J., Mckinnon, J. J., Wolff, S. K., Grimwood, D. J., Spackman, P. R., Jayatilaka, D. \& Spackman, M. A. (2017). Crystal Explorer 17. The University of Western Australia.

Vachon, J., Harthong, S., Jeanneau, E., Aronica, C., Vanthuyne, N., Roussel, C. \& Dutasta, J.-P. (2011). Org. Biomol. Chem. 9, 50865091.

Vircks, K. E. \& Mulligan, C. C. (2012). Rapid Commun. Mass Spectrom. 26, 2665-2672.

Westrip, S. P. (2010). J. Appl. Cryst. 43, 920-925.

Winstock, A. R., Marsden, J. \& Mitcheson, L. (2010). BMJ, 340, c1605.

Wood, D. M., Davies, S., Puchnarewicz, M., Button, J., Archer, R., Ovaska, H., Ramsey, J., Lee, T., Holt, D. W. \& Dargan, P. I. (2010). J. Med. Toxicol. 6, 327-330.

Wu, Y. L., Tancini, F., Schweizer, B. W., Paunescu, D., Boudon, C., Gisselbrecht, J.-P., Jarowski, P. D., Dalcanale, E. \& Diederich, F. (2012). Chem. Asian J. 7, 1185-1190. 


\section{supporting information}

Acta Cryst. (2019). E75, 277-283 [https://doi.org/10.1107/S2056989019001464]

\section{Crystal structure of a host-guest complex between mephedrone hydrochloride} and a tetraphosphonate cavitand

\section{Elisa Biavardi and Chiara Massera}

Computing details

Data collection: APEXII (Bruker, 2008); cell refinement: APEXII (Bruker, 2008); data reduction: SAINT (Bruker, 2008); program(s) used to solve structure: SIR97 (Altomare et al., 1999); program(s) used to refine structure: SHELXL2014/7 (Sheldrick, 2015); molecular graphics: Mercury (Macrae et al., 2006); software used to prepare material for publication: WinGX (Farrugia, 2012), PARST (Nardelli, 1995) and publCIF (Westrip, 2010).

Methyl[1-(4-methylphenyl)-1-oxopropan-2-yl]azanium chloride 2,8,14,20-tetrapropyl-5,11,17,23-

tetramethyl-6,10:12,16:18,22:24,4-tetrakis(phenylphosphonato- $O, O^{\prime}$ )resorcin[4] arene methanol monosolvate

Crystal data

$\mathrm{C}_{11} \mathrm{H}_{16} \mathrm{NO}^{+} \cdot \mathrm{Cl}^{-} \cdot \mathrm{C}_{68} \mathrm{H}_{68} \mathrm{O}_{12} \mathrm{P}_{4} \cdot \mathrm{CH}_{4} \mathrm{O}$

$F(000)=3056$

$M_{r}=1446.84$

Monoclinic, $P 2_{1} / c$

$D_{\mathrm{x}}=1.219 \mathrm{Mg} \mathrm{m}^{-3}$

$a=17.5353(8) \AA$

$b=22.4798$ (9) $\AA$

$c=21.2031$ (9) $\AA$

$\beta=109.455(1)^{\circ}$

$V=7880.8(6) \AA^{3}$

Mo $K \alpha$ radiation, $\lambda=0.71069 \AA$

$Z=4$

Cell parameters from 2781 reflections

$\theta=1.4-25.8^{\circ}$

$\mu=0.19 \mathrm{~mm}^{-1}$

$T=190 \mathrm{~K}$

Prismatic, colourless

$0.13 \times 0.10 \times 0.08 \mathrm{~mm}$

\section{Data collection}

Bruker APEXII CCD area-detector diffractometer

Radiation source: fine-focus sealed tube Graphite monochromator $\omega$-scan

Absorption correction: multi-scan

(SADABS; Bruker, 2008)

$T_{\min }=0.634, T_{\max }=0.745$

89697 measured reflections

15077 independent reflections

9535 reflections with $I>2 \sigma(I)$

$R_{\text {int }}=0.073$

$\theta_{\text {max }}=25.8^{\circ}, \theta_{\min }=1.4^{\circ}$

$h=-21 \rightarrow 21$

$k=-27 \rightarrow 27$

$l=-25 \rightarrow 25$

\section{Refinement}

Refinement on $F^{2}$

Least-squares matrix: full

$R\left[F^{2}>2 \sigma\left(F^{2}\right)\right]=0.077$

$w R\left(F^{2}\right)=0.275$

$S=1.02$

15077 reflections

929 parameters

2 restraints

Hydrogen site location: inferred from neighbouring sites

$\mathrm{H}$-atom parameters constrained

$w=1 /\left[\sigma^{2}\left(F_{\mathrm{o}}{ }^{2}\right)+(0.1708 P)^{2}+6.7275 P\right]$

where $P=\left(F_{\mathrm{o}}^{2}+2 F_{\mathrm{c}}^{2}\right) / 3$

$(\Delta / \sigma)_{\max }=0.001$

$\Delta \rho_{\max }=1.78 \mathrm{e} \AA^{-3}$

$\Delta \rho_{\min }=-0.52$ e $\AA^{-3}$ 


\section{Special details}

Geometry. All esds (except the esd in the dihedral angle between two 1.s. planes) are estimated using the full covariance matrix. The cell esds are taken into account individually in the estimation of esds in distances, angles and torsion angles; correlations between esds in cell parameters are only used when they are defined by crystal symmetry. An approximate (isotropic) treatment of cell esds is used for estimating esds involving l.s. planes.

Fractional atomic coordinates and isotropic or equivalent isotropic displacement parameters $\left(\AA^{2}\right)$

\begin{tabular}{|c|c|c|c|c|c|}
\hline & $x$ & $y$ & $z$ & $U_{\text {iso }} * / U_{\text {eq }}$ & Occ. $(<1)$ \\
\hline Cl1 & $0.08550(8)$ & $-0.09484(7)$ & $0.83375(7)$ & $0.0629(4)$ & \\
\hline N1 & $0.3301(2)$ & $0.10053(18)$ & $0.7202(2)$ & $0.0554(10)$ & \\
\hline $\mathrm{H} 1 \mathrm{~A}$ & 0.3375 & 0.1203 & 0.7593 & $0.066^{*}$ & \\
\hline H1B & 0.2979 & 0.1234 & 0.6864 & $0.066^{*}$ & \\
\hline $\mathrm{O} 1$ & $0.4139(3)$ & $0.19702(17)$ & $0.7037(2)$ & $0.0786(12)$ & \\
\hline $\mathrm{C} 1$ & $0.2883(3)$ & $0.0432(2)$ & $0.7216(3)$ & $0.0559(12)$ & \\
\hline $\mathrm{H} 1 \mathrm{C}$ & 0.2744 & 0.0239 & 0.6778 & $0.084 *$ & \\
\hline H1D & 0.2389 & 0.0508 & 0.7322 & $0.084 *$ & \\
\hline $\mathrm{H} 1 \mathrm{E}$ & 0.3242 & 0.0172 & 0.7559 & $0.084 *$ & \\
\hline $\mathrm{C} 2$ & $0.4103(3)$ & $0.0926(2)$ & $0.7107(3)$ & $0.0559(12)$ & \\
\hline $\mathrm{H} 2$ & 0.4429 & 0.0636 & 0.7448 & $0.067 *$ & \\
\hline $\mathrm{C} 3$ & $0.4012(5)$ & $0.0691(3)$ & $0.6436(4)$ & $0.094(2)$ & \\
\hline $\mathrm{H} 3 \mathrm{~A}$ & 0.3662 & 0.0340 & 0.6347 & $0.141^{*}$ & \\
\hline H3B & 0.4545 & 0.0581 & 0.6417 & $0.141^{*}$ & \\
\hline $\mathrm{H} 3 \mathrm{C}$ & 0.3770 & 0.0997 & 0.6099 & $0.141^{*}$ & \\
\hline $\mathrm{C} 4$ & $0.4542(3)$ & $0.1522(2)$ & $0.7228(3)$ & $0.0578(13)$ & \\
\hline $\mathrm{C} 5$ & $0.5426(3)$ & $0.1539(2)$ & $0.7557(3)$ & $0.0556(12)$ & \\
\hline C6 & $0.5884(4)$ & $0.1030(2)$ & $0.7777(3)$ & $0.0725(16)$ & \\
\hline H6 & 0.5632 & 0.0651 & 0.7702 & $0.087^{*}$ & \\
\hline $\mathrm{C} 7$ & $0.6700(4)$ & $0.1074(3)$ & $0.8101(4)$ & $0.086(2)$ & \\
\hline $\mathrm{H} 7$ & 0.7007 & 0.0721 & 0.8242 & $0.103 *$ & \\
\hline $\mathrm{C} 8$ & $0.7091(3)$ & $0.1621(3)$ & $0.8230(3)$ & $0.0669(15)$ & \\
\hline C9 & $0.6637(4)$ & $0.2117(3)$ & $0.7986(3)$ & $0.0778(17)$ & \\
\hline H9 & 0.6892 & 0.2495 & 0.8048 & $0.093^{*}$ & \\
\hline $\mathrm{C} 10$ & $0.5824(4)$ & $0.2080(2)$ & $0.7656(3)$ & $0.0743(17)$ & \\
\hline H10 & 0.5526 & 0.2433 & 0.7491 & $0.089^{*}$ & \\
\hline C11 & $0.7975(4)$ & $0.1679(3)$ & $0.8644(4)$ & $0.097(2)$ & \\
\hline H11A & 0.8306 & 0.1625 & 0.8356 & $0.145^{*}$ & \\
\hline H11B & 0.8117 & 0.1375 & 0.8995 & $0.145^{*}$ & \\
\hline $\mathrm{H} 11 \mathrm{C}$ & 0.8074 & 0.2075 & 0.8849 & $0.145^{*}$ & \\
\hline P1A & $0.38511(7)$ & $0.16364(5)$ & $0.90714(5)$ & 0.0380 & \\
\hline O1A & $0.40936(17)$ & $0.10664(12)$ & $0.95332(13)$ & $0.0384(6)$ & \\
\hline $\mathrm{O} 2 \mathrm{~A}$ & $0.29380(17)$ & $0.17777(13)$ & $0.90051(14)$ & $0.0433(7)$ & \\
\hline $\mathrm{O} 3 \mathrm{~A}$ & $0.39674(18)$ & $0.15745(13)$ & $0.84232(14)$ & $0.0449(7)$ & \\
\hline $\mathrm{P} 1 \mathrm{~B}$ & $0.10885(7)$ & $0.17333(5)$ & $0.61816(6)$ & $0.0407(3)$ & \\
\hline O1B & $0.08989(17)$ & $0.18649(13)$ & $0.68474(15)$ & $0.0427(7)$ & \\
\hline $\mathrm{O} 2 \mathrm{~B}$ & $0.04768(17)$ & $0.12187(12)$ & $0.58070(14)$ & $0.0407(7)$ & \\
\hline O3B & $0.19354(18)$ & $0.15819(13)$ & $0.62903(16)$ & $0.0475(7)$ & \\
\hline $\mathrm{P} 1 \mathrm{C}$ & $0.21929(7)$ & $-0.08274(5)$ & $0.52556(6)$ & $0.0417(3)$ & \\
\hline
\end{tabular}




\begin{tabular}{|c|c|c|c|c|c|}
\hline $\mathrm{O} 1 \mathrm{C}$ & $0.12856(17)$ & $-0.06563(13)$ & $0.51623(14)$ & $0.0404(7)$ & \\
\hline $\mathrm{O} 2 \mathrm{C}$ & $0.23930(17)$ & $-0.13912(13)$ & $0.57372(14)$ & $0.0417(7)$ & \\
\hline $\mathrm{O} 3 \mathrm{C}$ & $0.2765(2)$ & $-0.03344(15)$ & $0.54850(17)$ & $0.0556(8)$ & \\
\hline P1D & $0.50908(6)$ & $-0.08324(5)$ & $0.81214(5)$ & $0.0354(3)$ & \\
\hline O1D & $0.45599(16)$ & $-0.14052(12)$ & $0.78262(14)$ & $0.0375(6)$ & \\
\hline $\mathrm{O} 2 \mathrm{D}$ & $0.50278(16)$ & $-0.07416(12)$ & $0.88471(13)$ & $0.0355(6)$ & \\
\hline O3D & $0.48583(18)$ & $-0.03031(13)$ & $0.76989(15)$ & $0.0461(7)$ & \\
\hline C10D & $0.3303(3)$ & $-0.1689(2)$ & $0.9668(2)$ & $0.0537(12)$ & \\
\hline $\mathrm{H} 10 \mathrm{~K}$ & 0.3883 & -0.1796 & 0.9806 & $0.064 *$ & \\
\hline H10J & 0.3263 & -0.1285 & 0.9842 & $0.064 *$ & \\
\hline $\mathrm{C} 2 \mathrm{~A}$ & $0.3159(2)$ & $0.02458(18)$ & $0.91576(19)$ & $0.0362(9)$ & \\
\hline $\mathrm{C} 3 \mathrm{~A}$ & $0.3928(2)$ & $0.04831(18)$ & $0.92624(19)$ & $0.0355(9)$ & \\
\hline $\mathrm{C} 4 \mathrm{~A}$ & $0.4552(2)$ & $0.01803(17)$ & $0.91511(18)$ & $0.0336(9)$ & \\
\hline $\mathrm{C} 5 \mathrm{~A}$ & $0.4385(2)$ & $-0.04078(17)$ & $0.89356(19)$ & $0.0342(9)$ & \\
\hline C6A & $0.3637(2)$ & $-0.06805(17)$ & $0.88228(18)$ & $0.0331(9)$ & \\
\hline C7A & $0.5366(2)$ & $0.04572(19)$ & $0.9266(2)$ & $0.0414(10)$ & \\
\hline H7A1 & 0.5315 & 0.0781 & 0.8946 & $0.062 *$ & \\
\hline H7A2 & 0.5740 & 0.0156 & 0.9206 & $0.062 *$ & \\
\hline H7A3 & 0.5575 & 0.0615 & 0.9723 & $0.062 *$ & \\
\hline C8A & $0.2484(2)$ & $0.0611(2)$ & $0.9277(2)$ & $0.0403(10)$ & \\
\hline H8A & 0.2747 & 0.0916 & 0.9625 & $0.048^{*}$ & \\
\hline C9A & $0.1926(3)$ & $0.0241(3)$ & $0.9539(2)$ & $0.0570(13)$ & 0.5 \\
\hline H9A1 & 0.1650 & -0.0048 & 0.9184 & $0.068 *$ & 0.5 \\
\hline H9A2 & 0.2276 & 0.0007 & 0.9922 & $0.068^{*}$ & 0.5 \\
\hline $\mathrm{C} 10 \mathrm{~A}$ & $0.1322(6)$ & $0.0507(4)$ & $0.9750(5)$ & $0.049(2)^{*}$ & 0.5 \\
\hline H10A & 0.0934 & 0.0712 & 0.9361 & $0.059 *$ & 0.5 \\
\hline $\mathrm{H} 10 \mathrm{~B}$ & 0.1581 & 0.0815 & 1.0087 & $0.059 *$ & 0.5 \\
\hline C11A & $0.0858(8)$ & $0.0104(6)$ & $1.0041(7)$ & $0.079(3)^{*}$ & 0.5 \\
\hline H11D & 0.0636 & -0.0226 & 0.9731 & $0.119^{*}$ & 0.5 \\
\hline H11E & 0.0415 & 0.0324 & 1.0117 & $0.119^{*}$ & 0.5 \\
\hline $\mathrm{H} 11 \mathrm{~F}$ & 0.1217 & -0.0055 & 1.0467 & $0.119^{*}$ & 0.5 \\
\hline C9E & $0.1926(3)$ & $0.0241(3)$ & $0.9539(2)$ & $0.0570(13)$ & 0.5 \\
\hline H9E1 & 0.1444 & 0.0483 & 0.9512 & $0.068^{*}$ & 0.5 \\
\hline H9E2 & 0.1738 & -0.0108 & 0.9242 & $0.068^{*}$ & 0.5 \\
\hline C10E & $0.2270(7)$ & $0.0042(5)$ & $1.0187(5)$ & $0.060(3)^{*}$ & 0.5 \\
\hline H10C & 0.2530 & 0.0378 & 1.0481 & $0.072^{*}$ & 0.5 \\
\hline H10D & 0.2692 & -0.0258 & 1.0207 & $0.072^{*}$ & 0.5 \\
\hline C11E & $0.1624(9)$ & $-0.0237(7)$ & $1.0435(7)$ & $0.093(4)^{*}$ & 0.5 \\
\hline $\mathrm{H} 11 \mathrm{G}$ & 0.1221 & 0.0065 & 1.0435 & $0.139 *$ & 0.5 \\
\hline $\mathrm{H} 11 \mathrm{H}$ & 0.1879 & -0.0389 & 1.0890 & $0.139 *$ & 0.5 \\
\hline H11I & 0.1359 & -0.0564 & 1.0138 & $0.139 *$ & 0.5 \\
\hline $\mathrm{C} 12 \mathrm{~A}$ & $0.4380(3)$ & $0.22140(18)$ & $0.9610(2)$ & $0.0413(10)$ & \\
\hline C13A & $0.4582(4)$ & $0.2721(3)$ & $0.9337(3)$ & 0.0745 (18) & \\
\hline $\mathrm{H} 13 \mathrm{~A}$ & 0.4484 & 0.2742 & 0.8870 & $0.089 *$ & \\
\hline C14A & $0.4927(5)$ & $0.3200(3)$ & $0.9744(4)$ & $0.087(2)$ & \\
\hline H14A & 0.5028 & 0.3561 & 0.9552 & $0.105^{*}$ & \\
\hline $\mathrm{C} 15 \mathrm{~A}$ & $0.5122(4)$ & $0.3151(3)$ & $1.0423(3)$ & 0.0742 (17) & \\
\hline $\mathrm{H} 15 \mathrm{~A}$ & 0.5395 & 0.3468 & 1.0705 & $0.089 *$ & \\
\hline
\end{tabular}




\begin{tabular}{|c|c|c|c|c|}
\hline $\mathrm{C} 16 \mathrm{~A}$ & $0.4927(5)$ & $0.2649(3)$ & $1.0695(3)$ & $0.084(2)$ \\
\hline H16A & 0.5048 & 0.2623 & 1.1166 & $0.101^{*}$ \\
\hline $\mathrm{C} 17 \mathrm{~A}$ & $0.4551(4)$ & $0.2178(2)$ & 1.0288 & $0.0710(17)$ \\
\hline H17A & 0.4412 & 0.1830 & 1.0480 & $0.085^{*}$ \\
\hline $\mathrm{C} 1 \mathrm{~B}$ & $0.1377(2)$ & $0.06971(19)$ & $0.8132(2)$ & $0.0378(9)$ \\
\hline H1B1 & 0.1192 & 0.0314 & 0.8203 & $0.045^{*}$ \\
\hline $\mathrm{C} 2 \mathrm{~B}$ & $0.0980(2)$ & 0.09968 (19) & $0.7532(2)$ & $0.0383(9)$ \\
\hline C3B & $0.1283(2)$ & $0.15481(19)$ & $0.7442(2)$ & $0.0401(10)$ \\
\hline $\mathrm{C} 4 \mathrm{~B}$ & $0.1936(3)$ & $0.18243(19)$ & $0.7916(2)$ & $0.0433(10)$ \\
\hline $\mathrm{C} 5 \mathrm{~B}$ & $0.2294(2)$ & $0.1501(2)$ & $0.8505(2)$ & $0.0409(10)$ \\
\hline C6B & $0.2035(2)$ & 0.09445 (19) & $0.8628(2)$ & $0.0384(9)$ \\
\hline C7B & $0.2230(3)$ & $0.2430(2)$ & $0.7796(3)$ & $0.0528(12)$ \\
\hline H7B1 & 0.2473 & 0.2634 & 0.8226 & $0.079 *$ \\
\hline H7B2 & 0.1773 & 0.2665 & 0.7513 & $0.079 *$ \\
\hline H7B3 & 0.2635 & 0.2387 & 0.7574 & $0.079 *$ \\
\hline $\mathrm{C} 8 \mathrm{~B}$ & $0.0260(2)$ & $0.07312(19)$ & $0.7000(2)$ & $0.0389(9)$ \\
\hline H8B & -0.0063 & 0.1069 & 0.6737 & $0.047^{*}$ \\
\hline C9B & $-0.0300(3)$ & $0.0374(2)$ & $0.7276(2)$ & $0.0449(10)$ \\
\hline H9B1 & -0.0763 & 0.0228 & 0.6896 & $0.054 *$ \\
\hline H9B2 & -0.0003 & 0.0023 & 0.7518 & $0.054^{*}$ \\
\hline C10B & $-0.0624(3)$ & $0.0726(2)$ & $0.7747(3)$ & $0.0586(13)$ \\
\hline H10E & -0.0903 & 0.1089 & 0.7518 & $0.070^{*}$ \\
\hline $\mathrm{H} 10 \mathrm{~F}$ & -0.0170 & 0.0851 & 0.8146 & $0.070^{*}$ \\
\hline C11B & $-0.1216(5)$ & $0.0344(3)$ & 0.7964 & $0.100(2)$ \\
\hline H11L & -0.1605 & 0.0158 & 0.7569 & $0.150^{*}$ \\
\hline H11M & -0.1506 & 0.0596 & 0.8187 & $0.150 *$ \\
\hline $\mathrm{H} 11 \mathrm{~N}$ & -0.0917 & 0.0035 & 0.8274 & $0.150^{*}$ \\
\hline C12B & 0.0698 & $0.23712(19)$ & $0.5690(2)$ & $0.0447(10)$ \\
\hline C13B & $0.0186(3)$ & $0.2771(2)$ & $0.5852(3)$ & $0.0543(12)$ \\
\hline H13B & 0.0028 & 0.2702 & 0.6232 & $0.065^{*}$ \\
\hline C14B & -0.0093 & $0.3272(2)$ & $0.5457(3)$ & $0.0684(15)$ \\
\hline H14B & -0.0448 & 0.3543 & 0.5563 & $0.082 *$ \\
\hline C15B & $0.0146(4)$ & $0.3371(3)$ & $0.4913(3)$ & $0.0713(16)$ \\
\hline H15B & -0.0050 & 0.3712 & 0.4642 & $0.086^{*}$ \\
\hline $\mathrm{C} 16 \mathrm{~B}$ & 0.0660 & $0.2989(3)$ & $0.4751(3)$ & 0.0773 \\
\hline H16B & 0.0826 & 0.3068 & 0.4376 & $0.093^{*}$ \\
\hline C17B & $0.0939(4)$ & $0.2482(2)$ & $0.5142(3)$ & $0.0661(15)$ \\
\hline H17B & 0.1294 & 0.2214 & 0.5032 & $0.079 *$ \\
\hline $\mathrm{C} 1 \mathrm{C}$ & $0.0744(2)$ & $-0.02396(18)$ & $0.6637(2)$ & $0.0362(9)$ \\
\hline $\mathrm{H} 1 \mathrm{C} 1$ & 0.0697 & -0.0417 & 0.7028 & $0.043^{*}$ \\
\hline $\mathrm{C} 2 \mathrm{C}$ & $0.0996(2)$ & $-0.05894(18)$ & $0.6198(2)$ & $0.0362(9)$ \\
\hline $\mathrm{C} 3 \mathrm{C}$ & $0.1080(2)$ & $-0.03113(18)$ & 0.56427 (19) & $0.0354(9)$ \\
\hline $\mathrm{C} 4 \mathrm{C}$ & $0.0937(2)$ & $0.02916(19)$ & $0.5505(2)$ & $0.0384(9)$ \\
\hline $\mathrm{C} 5 \mathrm{C}$ & $0.0667(2)$ & $0.06113(18)$ & $0.5952(2)$ & $0.0362(9)$ \\
\hline $\mathrm{C} 6 \mathrm{C}$ & $0.0560(2)$ & $0.03618(18)$ & $0.6517(2)$ & $0.0367(9)$ \\
\hline C7C & $0.1049(3)$ & $0.0577(2)$ & $0.4899(2)$ & $0.0440(10)$ \\
\hline $\mathrm{H} 7 \mathrm{C} 1$ & 0.0730 & 0.0943 & 0.4790 & $0.066^{*}$ \\
\hline $\mathrm{H} 7 \mathrm{C} 2$ & 0.0868 & 0.0302 & 0.4520 & $0.066^{*}$ \\
\hline
\end{tabular}




\begin{tabular}{|c|c|c|c|c|}
\hline $\mathrm{H} 7 \mathrm{C} 3$ & 0.1622 & 0.0671 & 0.4994 & $0.066^{*}$ \\
\hline $\mathrm{C} 8 \mathrm{C}$ & $0.1184(2)$ & $-0.12493(18)$ & $0.6345(2)$ & $0.0367(9)$ \\
\hline $\mathrm{H} 8 \mathrm{C}$ & 0.1102 & -0.1449 & 0.5907 & $0.044^{*}$ \\
\hline $\mathrm{C} 9 \mathrm{C}$ & $0.0606(3)$ & $-0.15446(19)$ & $0.6653(2)$ & $0.0437(10)$ \\
\hline $\mathrm{H} 9 \mathrm{C} 1$ & 0.0689 & -0.1364 & 0.7097 & $0.052 *$ \\
\hline $\mathrm{H} 9 \mathrm{C} 2$ & 0.0043 & -0.1457 & 0.6368 & $0.052 *$ \\
\hline $\mathrm{C} 10 \mathrm{C}$ & $0.0702(3)$ & $-0.2207(2)$ & $0.6736(3)$ & $0.0529(12)$ \\
\hline $\mathrm{H} 10 \mathrm{H}$ & 0.1250 & -0.2298 & 0.7048 & $0.063^{*}$ \\
\hline H10G & 0.0649 & -0.2389 & 0.6299 & $0.063^{*}$ \\
\hline $\mathrm{C} 11 \mathrm{C}$ & $0.0071(3)$ & $-0.2479(2)$ & $0.7006(3)$ & $0.0671(15)$ \\
\hline $\mathrm{H} 11 \mathrm{O}$ & 0.0153 & -0.2327 & 0.7457 & $0.101^{*}$ \\
\hline H11P & 0.0128 & -0.2913 & 0.7023 & $0.101 *$ \\
\hline H11Q & -0.0473 & -0.2372 & 0.6711 & $0.101^{*}$ \\
\hline $\mathrm{C} 12 \mathrm{C}$ & $0.2126(3)$ & $-0.1168(2)$ & $0.4482(2)$ & $0.0498(11)$ \\
\hline $\mathrm{C} 13 \mathrm{C}$ & $0.2679(4)$ & $-0.1017(3)$ & $0.4170(3)$ & $0.0715(16)$ \\
\hline $\mathrm{H} 13 \mathrm{C}$ & 0.3060 & -0.0708 & 0.4343 & $0.086^{*}$ \\
\hline $\mathrm{C} 14 \mathrm{C}$ & $0.2664(4)$ & $-0.1332(3)$ & $0.3590(3)$ & 0.0816 (19) \\
\hline $\mathrm{H} 14 \mathrm{C}$ & 0.3040 & -0.1235 & 0.3370 & $0.098^{*}$ \\
\hline $\mathrm{C} 15 \mathrm{C}$ & $0.2118(4)$ & $-0.1771(3)$ & $0.3345(3)$ & $0.0738(18)$ \\
\hline $\mathrm{H} 15 \mathrm{C}$ & 0.2117 & -0.1980 & 0.2955 & $0.089^{*}$ \\
\hline $\mathrm{C} 16 \mathrm{C}$ & $0.1580(4)$ & $-0.1916(3)$ & 0.3643 & $0.0727(16)$ \\
\hline $\mathrm{H} 16 \mathrm{C}$ & 0.1198 & -0.2223 & 0.3460 & $0.087^{*}$ \\
\hline $\mathrm{C} 17 \mathrm{C}$ & $0.1574(3)$ & $-0.1621(2)$ & $0.4216(2)$ & $0.0570(13)$ \\
\hline $\mathrm{H} 17 \mathrm{C}$ & 0.1193 & -0.1729 & 0.4427 & $0.068^{*}$ \\
\hline C1D & $0.2356(2)$ & $-0.13048(17)$ & $0.7459(2)$ & $0.0361(9)$ \\
\hline H1D1 & 0.1975 & -0.1283 & 0.7689 & $0.043^{*}$ \\
\hline $\mathrm{C} 2 \mathrm{D}$ & $0.3179(2)$ & $-0.13337(17)$ & $0.7825(2)$ & $0.0346(9)$ \\
\hline C3D & $0.3721(2)$ & $-0.13563(17)$ & $0.7472(2)$ & $0.0370(9)$ \\
\hline C4D & $0.3488(2)$ & $-0.13616(19)$ & $0.6780(2)$ & $0.0384(9)$ \\
\hline C5D & $0.2659(3)$ & $-0.13406(18)$ & $0.6444(2)$ & $0.0385(9)$ \\
\hline C6D & $0.2079(2)$ & $-0.13078(17)$ & $0.6756(2)$ & $0.0366(9)$ \\
\hline C7D & $0.4084(3)$ & $-0.1409(2)$ & $0.6415(2)$ & $0.0512(12)$ \\
\hline H7D1 & 0.3815 & -0.1572 & 0.5967 & $0.077^{*}$ \\
\hline H7D2 & 0.4529 & -0.1672 & 0.6662 & $0.077^{*}$ \\
\hline H7D3 & 0.4300 & -0.1013 & 0.6376 & $0.077^{*}$ \\
\hline C8D & $0.3491(3)$ & $-0.13214(18)$ & $0.8587(2)$ & $0.0370(9)$ \\
\hline H8D & 0.4032 & -0.1521 & 0.8732 & $0.044 *$ \\
\hline C9D & $0.2954(3)$ & $-0.1677(2)$ & $0.8902(2)$ & $0.0447(10)$ \\
\hline H9D1 & 0.2408 & -0.1497 & 0.8762 & $0.054 *$ \\
\hline H9D2 & 0.2899 & -0.2090 & 0.8731 & $0.054 *$ \\
\hline C1A & $0.3029(2)$ & -0.03379 (19) & $0.89266(19)$ & $0.0373(9)$ \\
\hline H1A1 & 0.2507 & -0.0508 & 0.8837 & $0.045^{*}$ \\
\hline C11D & $0.2872(5)$ & $-0.2126(3)$ & 0.9978 & $0.088(2)$ \\
\hline H11R & 0.2305 & -0.2006 & 0.9869 & $0.133^{*}$ \\
\hline H11S & 0.3135 & -0.2128 & 1.0465 & $0.133^{*}$ \\
\hline $\mathrm{H} 11 \mathrm{~T}$ & 0.2899 & -0.2525 & 0.9800 & $0.133^{*}$ \\
\hline C12D & $0.6095(3)$ & -0.11008 (19) & $0.8291(2)$ & $0.0403(9)$ \\
\hline C13D & $0.6686(4)$ & $-0.0701(3)$ & $0.8263(4)$ & $0.084(2)$ \\
\hline
\end{tabular}




$\begin{array}{llllll}\text { H13D } & 0.6555 & -0.0293 & 0.8174 & 0.101^{*} & \\ \text { C14D } & 0.7447(4) & -0.0892(3) & 0.8361(5) & 0.100(3) & \\ \text { H14D } & 0.7837 & -0.0619 & 0.8312 & 0.120^{*} & \\ \text { C15D } & 0.7671(3) & -0.1469(3) & 0.8528(3) & 0.0649(15) & \\ \text { H15D } & 0.8217 & -0.1590 & 0.8629 & 0.078^{*} & \\ \text { C16D } & 0.7105(3) & -0.1858(3) & 0.8548(3) & 0.0718(16) & \\ \text { H16D } & 0.7248 & -0.2262 & 0.8655 & 0.086^{*} & \\ \text { C17D } & 0.6309(3) & -0.1678(2) & 0.8413(3) & 0.0604(14) & \\ \text { H17D } & 0.5909 & -0.1965 & 0.8406 & 0.072^{*} & \\ \text { O1S } & 0.5707(10) & 0.0241(7) & 0.6032(8) & 0.103(5)^{*} & 0.335(6) \\ \text { H1S } & 0.5450 & 0.0115 & 0.5647 & 0.154^{*} & 0.335(6) \\ \text { C1S } & 0.6098(12) & -0.0253(8) & 0.6455(11) & 0.105(8) & 0.335(6) \\ \text { H1S1 } & 0.6675 & -0.0167 & 0.6666 & 0.158^{*} & 0.335(6) \\ \text { H1S2 } & 0.6033 & -0.0615 & 0.6184 & 0.158^{*} & 0.335(6) \\ \text { H1S3 } & 0.5851 & -0.0313 & 0.6802 & 0.158^{*} & 0.335(6) \\ \text { O2S } & -0.0947(3) & -0.1331(3) & 0.7768(3) & 0.0699(19) & 0.665(6) \\ \text { H2S } & -0.0485 & -0.1178 & 0.7930 & 0.105^{*} & 0.665(6) \\ \text { C2S } & -0.1305(5) & -0.1142(4) & 0.7091(4) & 0.075(3) & 0.665(6) \\ \text { H2S1 } & -0.1836 & -0.0967 & 0.7029 & 0.113^{*} & 0.665(6) \\ \text { H2S2 } & -0.1367 & -0.1485 & 0.6793 & 0.113^{*} & 0.665(6) \\ \text { H2S3 } & -0.0956 & -0.0845 & 0.6985 & 0.113^{*} & 0.665(6)\end{array}$

Atomic displacement parameters $\left(\AA^{2}\right)$

\begin{tabular}{lllllll}
\hline & $U^{11}$ & $U^{22}$ & $U^{33}$ & $U^{12}$ & $U^{13}$ & $U^{23}$ \\
\hline C11 & $0.0503(7)$ & $0.0783(9)$ & $0.0617(8)$ & $-0.0120(6)$ & $0.0208(6)$ & $-0.0002(7)$ \\
N1 & $0.052(2)$ & $0.053(2)$ & $0.058(2)$ & $0.0033(19)$ & $0.013(2)$ & $-0.0020(19)$ \\
O1 & $0.083(3)$ & $0.053(2)$ & $0.095(3)$ & $0.008(2)$ & $0.024(2)$ & $0.013(2)$ \\
C1 & $0.046(3)$ & $0.051(3)$ & $0.072(3)$ & $0.000(2)$ & $0.021(2)$ & $0.002(2)$ \\
C2 & $0.053(3)$ & $0.050(3)$ & $0.066(3)$ & $0.004(2)$ & $0.021(2)$ & $-0.008(2)$ \\
C3 & $0.107(6)$ & $0.081(5)$ & $0.101(5)$ & $-0.029(4)$ & $0.042(4)$ & $-0.035(4)$ \\
C4 & $0.071(3)$ & $0.045(3)$ & $0.061(3)$ & $0.007(3)$ & $0.026(3)$ & $0.007(2)$ \\
C5 & $0.061(3)$ & $0.047(3)$ & $0.062(3)$ & $-0.002(2)$ & $0.025(3)$ & $0.000(2)$ \\
C6 & $0.067(4)$ & $0.042(3)$ & $0.111(5)$ & $-0.002(3)$ & $0.035(3)$ & $0.000(3)$ \\
C7 & $0.052(3)$ & $0.054(3)$ & $0.147(6)$ & $0.001(3)$ & $0.027(4)$ & $0.014(4)$ \\
C8 & $0.062(3)$ & $0.059(3)$ & $0.085(4)$ & $-0.008(3)$ & $0.031(3)$ & $-0.002(3)$ \\
C9 & $0.079(4)$ & $0.054(3)$ & $0.092(4)$ & $-0.016(3)$ & $0.017(3)$ & $-0.001(3)$ \\
C10 & $0.081(4)$ & $0.041(3)$ & $0.088(4)$ & $-0.009(3)$ & $0.010(3)$ & $-0.001(3)$ \\
C11 & $0.063(4)$ & $0.083(5)$ & $0.145(7)$ & $-0.019(3)$ & $0.035(4)$ & $-0.011(4)$ \\
P1A & $0.0383(6)$ & $0.0354(6)$ & $0.0377(6)$ & $-0.0013(5)$ & $0.0090(5)$ & $-0.0070(4)$ \\
O1A & $0.0392(15)$ & $0.0342(15)$ & $0.0383(15)$ & $-0.0018(12)$ & $0.0082(12)$ & $-0.0061(12)$ \\
O2A & $0.0382(16)$ & $0.0421(17)$ & $0.0453(17)$ & $0.0010(13)$ & $0.0081(13)$ & $-0.0092(13)$ \\
O3A & $0.0487(18)$ & $0.0447(17)$ & $0.0395(16)$ & $-0.0002(14)$ & $0.0122(13)$ & $-0.0051(13)$ \\
P1B & $0.0378(6)$ & $0.0340(6)$ & $0.0476(6)$ & $0.0005(5)$ & $0.0107(5)$ & $0.0004(5)$ \\
O1B & $0.0407(16)$ & $0.0365(15)$ & $0.0467(17)$ & $0.0025(13)$ & $0.0089(13)$ & $-0.0001(13)$ \\
O2B & $0.0400(16)$ & $0.0313(15)$ & $0.0455(16)$ & $0.0029(12)$ & $0.0073(13)$ & $0.0007(12)$ \\
O3B & $0.0395(16)$ & $0.0416(17)$ & $0.0597(19)$ & $0.0006(13)$ & $0.0143(14)$ & $0.0013(14)$ \\
P1C & $0.0397(6)$ & $0.0435(6)$ & $0.0386(6)$ & $0.0003(5)$ & $0.0085(5)$ & $-0.0026(5)$ \\
& & & & & &
\end{tabular}




\begin{tabular}{|c|c|c|c|c|c|c|}
\hline $\mathrm{O} 1 \mathrm{C}$ & $0.0395(16)$ & $0.0388(16)$ & $0.0377(15)$ & $0.0004(13)$ & $0.0061(12)$ & $-0.0054(12)$ \\
\hline $\mathrm{O} 2 \mathrm{C}$ & $0.0415(16)$ & $0.0445(17)$ & $0.0347(15)$ & $0.0036(13)$ & $0.0070(12)$ & $-0.0047(13)$ \\
\hline $\mathrm{O} 3 \mathrm{C}$ & $0.0477(18)$ & $0.054(2)$ & $0.061(2)$ & $-0.0095(16)$ & $0.0119(16)$ & $-0.0028(16)$ \\
\hline P1D & $0.0342(5)$ & $0.0300(5)$ & $0.0391(6)$ & $0.0011(4)$ & $0.0086(4)$ & $0.0033(4)$ \\
\hline O1D & $0.0314(14)$ & $0.0350(15)$ & $0.0401(15)$ & $0.0029(12)$ & $0.0040(12)$ & $0.0005(12)$ \\
\hline $\mathrm{O} 2 \mathrm{D}$ & $0.0322(14)$ & $0.0331(14)$ & $0.0373(15)$ & $0.0029(11)$ & $0.0065(12)$ & $0.0032(11)$ \\
\hline $\mathrm{O} 3 \mathrm{D}$ & $0.0518(18)$ & $0.0388(16)$ & $0.0449(17)$ & $0.0049(14)$ & $0.0123(14)$ & $0.0078(13)$ \\
\hline C10D & 0.064 (3) & $0.050(3)$ & $0.046(3)$ & $-0.012(2)$ & $0.018(2)$ & 0.007 (2) \\
\hline $\mathrm{C} 2 \mathrm{~A}$ & $0.037(2)$ & $0.040(2)$ & $0.0301(19)$ & $-0.0011(18)$ & $0.0091(17)$ & $0.0005(17)$ \\
\hline $\mathrm{C} 3 \mathrm{~A}$ & $0.037(2)$ & $0.033(2)$ & $0.034(2)$ & $-0.0050(17)$ & $0.0080(17)$ & $-0.0011(16)$ \\
\hline $\mathrm{C} 4 \mathrm{~A}$ & $0.036(2)$ & $0.033(2)$ & $0.0300(19)$ & $-0.0030(17)$ & $0.0079(16)$ & $0.0005(16)$ \\
\hline $\mathrm{C} 5 \mathrm{~A}$ & $0.037(2)$ & $0.033(2)$ & $0.0309(19)$ & $0.0009(17)$ & $0.0096(17)$ & $0.0044(16)$ \\
\hline C6A & $0.035(2)$ & $0.033(2)$ & $0.0283(19)$ & $-0.0050(17)$ & $0.0060(16)$ & $0.0031(16)$ \\
\hline C7A & $0.036(2)$ & $0.038(2)$ & $0.049(2)$ & $-0.0051(18)$ & $0.0124(19)$ & $-0.0031(19)$ \\
\hline $\mathrm{C} 8 \mathrm{~A}$ & $0.034(2)$ & $0.051(3)$ & $0.037(2)$ & $-0.0044(19)$ & $0.0141(18)$ & $-0.0066(19)$ \\
\hline C9A & $0.055(3)$ & $0.078(4)$ & $0.043(3)$ & $-0.011(3)$ & $0.023(2)$ & $-0.007(2)$ \\
\hline C9E & $0.055(3)$ & $0.078(4)$ & $0.043(3)$ & $-0.011(3)$ & $0.023(2)$ & $-0.007(2)$ \\
\hline $\mathrm{C} 12 \mathrm{~A}$ & $0.040(2)$ & $0.033(2)$ & $0.049(2)$ & $-0.0010(18)$ & $0.0126(19)$ & $-0.0100(19)$ \\
\hline $\mathrm{C} 13 \mathrm{~A}$ & $0.112(5)$ & $0.067(4)$ & $0.061(3)$ & $-0.040(3)$ & $0.051(3)$ & $-0.025(3)$ \\
\hline C14A & $0.124(6)$ & $0.064(4)$ & $0.099(5)$ & -0.049 (4) & $0.071(5)$ & $-0.030(3)$ \\
\hline $\mathrm{C} 15 \mathrm{~A}$ & $0.078(4)$ & $0.057(3)$ & $0.088(4)$ & $-0.018(3)$ & $0.028(3)$ & $-0.035(3)$ \\
\hline $\mathrm{C} 16 \mathrm{~A}$ & $0.124(6)$ & $0.060(4)$ & $0.052(3)$ & $-0.013(4)$ & $0.007(3)$ & $-0.018(3)$ \\
\hline C17A & $0.109(5)$ & $0.043(3)$ & $0.044(3)$ & $-0.005(3)$ & $0.004(3)$ & $-0.002(2)$ \\
\hline $\mathrm{C} 1 \mathrm{~B}$ & $0.033(2)$ & $0.041(2)$ & $0.043(2)$ & $0.0011(17)$ & $0.0161(18)$ & $-0.0032(18)$ \\
\hline $\mathrm{C} 2 \mathrm{~B}$ & $0.032(2)$ & $0.043(2)$ & $0.042(2)$ & $0.0030(18)$ & $0.0134(18)$ & $-0.0039(18)$ \\
\hline $\mathrm{C} 3 \mathrm{~B}$ & $0.035(2)$ & $0.041(2)$ & $0.042(2)$ & $0.0079(18)$ & $0.0099(18)$ & $-0.0019(19)$ \\
\hline $\mathrm{C} 4 \mathrm{~B}$ & $0.038(2)$ & $0.037(2)$ & $0.053(3)$ & $0.0032(18)$ & $0.012(2)$ & $-0.006(2)$ \\
\hline $\mathrm{C} 5 \mathrm{~B}$ & $0.033(2)$ & $0.047(2)$ & $0.040(2)$ & $0.0035(19)$ & $0.0090(18)$ & $-0.0120(19)$ \\
\hline C6B & $0.034(2)$ & $0.043(2)$ & $0.039(2)$ & $0.0030(18)$ & $0.0132(18)$ & $-0.0050(18)$ \\
\hline C7B & $0.053(3)$ & $0.034(2)$ & $0.064(3)$ & $0.000(2)$ & $0.009(2)$ & $-0.003(2)$ \\
\hline $\mathrm{C} 8 \mathrm{~B}$ & $0.033(2)$ & $0.036(2)$ & $0.047(2)$ & $0.0015(17)$ & $0.0130(18)$ & $0.0020(18)$ \\
\hline C9B & $0.035(2)$ & $0.046(2)$ & $0.053(3)$ & $0.0001(19)$ & $0.013(2)$ & $0.004(2)$ \\
\hline $\mathrm{C} 10 \mathrm{~B}$ & $0.051(3)$ & $0.057(3)$ & $0.076(3)$ & $0.011(2)$ & $0.031(3)$ & $0.010(3)$ \\
\hline $\mathrm{C} 11 \mathrm{~B}$ & $0.108(5)$ & $0.081(5)$ & $0.146(7)$ & $-0.001(4)$ & $0.089(5)$ & $0.009(5)$ \\
\hline $\mathrm{C} 12 \mathrm{~B}$ & $0.044(2)$ & $0.036(2)$ & $0.050(3)$ & $-0.0016(19)$ & $0.009(2)$ & $-0.0021(19)$ \\
\hline C13B & $0.053(3)$ & $0.044(3)$ & $0.065(3)$ & $0.007(2)$ & $0.019(2)$ & $0.002(2)$ \\
\hline C14B & $0.070(4)$ & $0.053(3)$ & $0.079(4)$ & $0.019(3)$ & $0.021(3)$ & $0.009(3)$ \\
\hline C15B & $0.093(4)$ & $0.050(3)$ & $0.059(3)$ & $0.019(3)$ & $0.009(3)$ & $0.010(3)$ \\
\hline $\mathrm{C} 16 \mathrm{~B}$ & $0.119(5)$ & $0.060(3)$ & $0.051(3)$ & $0.014(4)$ & $0.026(3)$ & $0.013(3)$ \\
\hline C17B & $0.097(4)$ & $0.044(3)$ & $0.063(3)$ & $0.013(3)$ & $0.034(3)$ & $0.004(2)$ \\
\hline $\mathrm{C} 1 \mathrm{C}$ & $0.0279(19)$ & $0.038(2)$ & $0.037(2)$ & $-0.0038(17)$ & $0.0036(17)$ & $0.0007(17)$ \\
\hline $\mathrm{C} 2 \mathrm{C}$ & $0.029(2)$ & $0.035(2)$ & $0.040(2)$ & $-0.0039(17)$ & $0.0040(17)$ & $-0.0018(17)$ \\
\hline $\mathrm{C} 3 \mathrm{C}$ & $0.033(2)$ & $0.037(2)$ & $0.032(2)$ & $-0.0024(17)$ & $0.0049(16)$ & $-0.0031(17)$ \\
\hline $\mathrm{C} 4 \mathrm{C}$ & $0.032(2)$ & $0.041(2)$ & $0.036(2)$ & $-0.0002(17)$ & $0.0016(17)$ & $0.0002(18)$ \\
\hline $\mathrm{C} 5 \mathrm{C}$ & $0.030(2)$ & $0.033(2)$ & $0.040(2)$ & $-0.0019(16)$ & $0.0035(17)$ & $0.0012(17)$ \\
\hline $\mathrm{C} 6 \mathrm{C}$ & $0.028(2)$ & $0.037(2)$ & $0.043(2)$ & $-0.0052(17)$ & $0.0081(17)$ & $-0.0042(18)$ \\
\hline $\mathrm{C} 7 \mathrm{C}$ & $0.046(2)$ & $0.042(2)$ & $0.041(2)$ & $-0.001(2)$ & $0.011(2)$ & $0.0046(19)$ \\
\hline $\mathrm{C} 8 \mathrm{C}$ & $0.035(2)$ & $0.033(2)$ & $0.038(2)$ & $-0.0013(17)$ & $0.0059(17)$ & $-0.0033(17)$ \\
\hline
\end{tabular}




$\begin{array}{lllllll}\text { C9C } & 0.037(2) & 0.040(2) & 0.049(3) & -0.0077(19) & 0.0072(19) & 0.0011(19) \\ \text { C10C } & 0.048(3) & 0.039(2) & 0.067(3) & -0.003(2) & 0.014(2) & 0.004(2) \\ \text { C11C } & 0.070(4) & 0.049(3) & 0.084(4) & -0.006(3) & 0.027(3) & 0.012(3) \\ \text { C12C } & 0.050(3) & 0.056(3) & 0.037(2) & 0.012(2) & 0.006(2) & 0.005(2) \\ \text { C13C } & 0.075(4) & 0.090(4) & 0.056(3) & 0.000(3) & 0.030(3) & 0.004(3) \\ \text { C14C } & 0.086(5) & 0.112(5) & 0.059(4) & 0.025(4) & 0.041(3) & 0.009(4) \\ \text { C15C } & 0.079(4) & 0.088(5) & 0.043(3) & 0.031(4) & 0.005(3) & -0.005(3) \\ \text { C16C } & 0.084(4) & 0.075(4) & 0.050(3) & 0.009(3) & 0.010(3) & -0.013(3) \\ \text { C17C } & 0.059(3) & 0.060(3) & 0.044(3) & 0.006(2) & 0.006(2) & -0.008(2) \\ \text { C1D } & 0.037(2) & 0.032(2) & 0.037(2) & -0.0037(17) & 0.0101(17) & 0.0004(17) \\ \text { C2D } & 0.034(2) & 0.030(2) & 0.037(2) & -0.0036(16) & 0.0073(17) & 0.0016(16) \\ \text { C3D } & 0.032(2) & 0.030(2) & 0.041(2) & -0.0013(17) & 0.0025(17) & 0.0007(17) \\ \text { C4D } & 0.037(2) & 0.038(2) & 0.038(2) & 0.0034(18) & 0.0102(18) & -0.0012(18) \\ \text { C5D } & 0.043(2) & 0.035(2) & 0.034(2) & 0.0042(18) & 0.0080(18) & 0.0009(17) \\ \text { C6D } & 0.037(2) & 0.0263(19) & 0.040(2) & -0.0029(17) & 0.0041(17) & -0.0001(16) \\ \text { C7D } & 0.043(3) & 0.062(3) & 0.047(3) & 0.008(2) & 0.013(2) & -0.005(2) \\ \text { C8D } & 0.040(2) & 0.031(2) & 0.037(2) & -0.0026(17) & 0.0076(18) & 0.0062(17) \\ \text { C9D } & 0.050(3) & 0.039(2) & 0.042(2) & -0.013(2) & 0.011(2) & 0.0041(19) \\ \text { C1A } & 0.036(2) & 0.040(2) & 0.034(2) & -0.0079(18) & 0.0086(17) & 0.0046(17) \\ \text { C11D } & 0.122(6) & 0.089(5) & 0.057(3) & -0.039(4) & 0.034(4) & 0.009(3) \\ \text { C12D } & 0.037(2) & 0.038(2) & 0.044(2) & 0.0008(18) & 0.0114(18) & -0.0003(18) \\ \text { C13D } & 0.058(3) & 0.048(3) & 0.160(7) & 0.006(3) & 0.054(4) & 0.025(4) \\ \text { C14D } & 0.058(4) & 0.076(4) & 0.183(8) & -0.001(3) & 0.061(5) & 0.032(5) \\ \text { C15D } & 0.041(3) & 0.067(4) & 0.087(4) & 0.006(3) & 0.021(3) & -0.007(3) \\ \text { C16D } & 0.046(3) & 0.051(3) & 0.113(5) & 0.009(3) & 0.019(3) & 0.008(3) \\ \text { C17D } & 0.045(3) & 0.039(3) & 0.095(4) & -0.001(2) & 0.021(3) & 0.008(3) \\ \text { C1S } & 0.095(16) & 0.099(17) & 0.15(2) & -0.026(13) & 0.078(16) & -0.010(16) \\ \text { O2S } & 0.037(3) & 0.080(4) & 0.094(5) & -0.021(3) & 0.024(3) & 0.001(3) \\ \text { C2S } & 0.057(5) & 0.054(5) & 0.120(8) & -0.015(4) & 0.035(5) & -0.010(5)\end{array}$

Geometric parameters $\left(\AA,{ }^{\circ}\right)$

\begin{tabular}{llll}
\hline $\mathrm{N} 1-\mathrm{C} 1$ & $1.488(6)$ & $\mathrm{C} 3 \mathrm{~B}-\mathrm{C} 4 \mathrm{~B}$ & $1.393(6)$ \\
$\mathrm{N} 1-\mathrm{C} 2$ & $1.495(6)$ & $\mathrm{C} 4 \mathrm{~B}-\mathrm{C} 5 \mathrm{~B}$ & $1.400(6)$ \\
$\mathrm{N} 1-\mathrm{H} 1 \mathrm{~A}$ & 0.9100 & $\mathrm{C} 4 \mathrm{~B}-\mathrm{C} 7 \mathrm{~B}$ & $1.507(6)$ \\
$\mathrm{N} 1-\mathrm{H} 1 \mathrm{~B}$ & 0.9100 & $\mathrm{C} 5 \mathrm{~B}-\mathrm{C} 6 \mathrm{~B}$ & 0.9800 \\
$\mathrm{O} 1-\mathrm{C} 4$ & $1.221(6)$ & $\mathrm{C} 7 \mathrm{~B}-\mathrm{H} 7 \mathrm{~B} 1$ & 0.9800 \\
$\mathrm{C} 1-\mathrm{H} 1 \mathrm{C}$ & 0.9800 & $\mathrm{C} 7 \mathrm{~B}-\mathrm{H} 7 \mathrm{~B} 2$ & 0.9800 \\
$\mathrm{C} 1-\mathrm{H} 1 \mathrm{D}$ & 0.9800 & $\mathrm{C} 7 \mathrm{~B}-\mathrm{H} 7 \mathrm{~B} 3$ & $1.528(6)$ \\
$\mathrm{C} 1-\mathrm{H} 1 \mathrm{E}$ & 0.9800 & $\mathrm{C} 8 \mathrm{~B}-\mathrm{C} 9 \mathrm{~B}$ & $1.541(6)$ \\
$\mathrm{C} 2-\mathrm{C} 3$ & $1.476(8)$ & $\mathrm{C} 8 \mathrm{~B}-\mathrm{C} 6 \mathrm{C}$ & 1.0000 \\
$\mathrm{C} 2-\mathrm{C} 4$ & $1.522(7)$ & $\mathrm{C} 8 \mathrm{~B}-\mathrm{H} 8 \mathrm{~B}$ & $1.525(7)$ \\
$\mathrm{C} 2-\mathrm{H} 2$ & 1.0000 & $\mathrm{C} 9 \mathrm{~B}-\mathrm{C} 10 \mathrm{~B}$ & 0.9900 \\
$\mathrm{C} 3-\mathrm{H} 3 \mathrm{~A}$ & 0.9800 & $\mathrm{C} 9 \mathrm{~B}-\mathrm{H} 9 \mathrm{~B} 1$ & 0.9900 \\
$\mathrm{C} 3-\mathrm{H} 3 \mathrm{~B}$ & 0.9800 & $\mathrm{C} 9 \mathrm{~B}-\mathrm{H} 9 \mathrm{~B} 2$ & $1.531(8)$ \\
$\mathrm{C} 3-\mathrm{H} 3 \mathrm{C}$ & 0.9800 & $\mathrm{C} 10 \mathrm{~B}-\mathrm{C} 11 \mathrm{~B}$ & 0.9900 \\
$\mathrm{C} 4-\mathrm{C} 5$ & $1.473(8)$ & $\mathrm{C} 10 \mathrm{~B}-\mathrm{H} 10 \mathrm{E}$ & 0.9900 \\
$\mathrm{C} 5-\mathrm{C} 10$ & $1.383(7)$ & $\mathrm{C} 10 \mathrm{~B}-\mathrm{H} 10 \mathrm{~F}$ &
\end{tabular}




\begin{tabular}{|c|c|c|c|}
\hline $\mathrm{C} 5-\mathrm{C} 6$ & $1.385(8)$ & $\mathrm{C} 11 \mathrm{~B}-\mathrm{H} 11 \mathrm{~L}$ & 0.9800 \\
\hline $\mathrm{C} 6-\mathrm{C} 7$ & $1.369(9)$ & $\mathrm{C} 11 \mathrm{~B}-\mathrm{H} 11 \mathrm{M}$ & 0.9800 \\
\hline $\mathrm{C} 6-\mathrm{H} 6$ & 0.9500 & $\mathrm{C} 11 \mathrm{~B}-\mathrm{H} 11 \mathrm{~N}$ & 0.9800 \\
\hline $\mathrm{C} 7-\mathrm{C} 8$ & $1.388(8)$ & $\mathrm{C} 12 \mathrm{~B}-\mathrm{C} 17 \mathrm{~B}$ & $1.385(7)$ \\
\hline $\mathrm{C} 7-\mathrm{H} 7$ & 0.9500 & $\mathrm{C} 12 \mathrm{~B}-\mathrm{C} 13 \mathrm{~B}$ & $1.391(7)$ \\
\hline $\mathrm{C} 8-\mathrm{C} 9$ & $1.367(8)$ & $\mathrm{C} 13 \mathrm{~B}-\mathrm{C} 14 \mathrm{~B}$ & $1.391(7)$ \\
\hline $\mathrm{C} 8-\mathrm{C} 11$ & $1.512(9)$ & $\mathrm{C} 13 \mathrm{~B}-\mathrm{H} 13 \mathrm{~B}$ & 0.9500 \\
\hline $\mathrm{C} 9-\mathrm{C} 10$ & $1.365(8)$ & $\mathrm{C} 14 \mathrm{~B}-\mathrm{C} 15 \mathrm{~B}$ & $1.370(8)$ \\
\hline $\mathrm{C} 9-\mathrm{H} 9$ & 0.9500 & $\mathrm{C} 14 \mathrm{~B}-\mathrm{H} 14 \mathrm{~B}$ & 0.9500 \\
\hline $\mathrm{C} 10-\mathrm{H} 10$ & 0.9500 & $\mathrm{C} 15 \mathrm{~B}-\mathrm{C} 16 \mathrm{~B}$ & $1.370(9)$ \\
\hline $\mathrm{C} 11-\mathrm{H} 11 \mathrm{~A}$ & 0.9800 & $\mathrm{C} 15 \mathrm{~B}-\mathrm{H} 15 \mathrm{~B}$ & 0.9500 \\
\hline C11-H11B & 0.9800 & $\mathrm{C} 16 \mathrm{~B}-\mathrm{C} 17 \mathrm{~B}$ & $1.398(7)$ \\
\hline $\mathrm{C} 11-\mathrm{H} 11 \mathrm{C}$ & 0.9800 & $\mathrm{C} 16 \mathrm{~B}-\mathrm{H} 16 \mathrm{~B}$ & 0.9500 \\
\hline $\mathrm{P} 1 \mathrm{~A}-\mathrm{O} 3 \mathrm{~A}$ & $1.462(3)$ & C17B-H17B & 0.9500 \\
\hline $\mathrm{P} 1 \mathrm{~A}-\mathrm{O} 1 \mathrm{~A}$ & $1.582(3)$ & $\mathrm{C} 1 \mathrm{C}-\mathrm{C} 6 \mathrm{C}$ & $1.393(6)$ \\
\hline $\mathrm{P} 1 \mathrm{~A}-\mathrm{O} 2 \mathrm{~A}$ & $1.592(3)$ & $\mathrm{C} 1 \mathrm{C}-\mathrm{C} 2 \mathrm{C}$ & $1.396(6)$ \\
\hline $\mathrm{P} 1 \mathrm{~A}-\mathrm{C} 12 \mathrm{~A}$ & $1.774(4)$ & $\mathrm{C} 1 \mathrm{C}-\mathrm{H} 1 \mathrm{C} 1$ & 0.9500 \\
\hline $\mathrm{O} 1 \mathrm{~A}-\mathrm{C} 3 \mathrm{~A}$ & $1.422(5)$ & $\mathrm{C} 2 \mathrm{C}-\mathrm{C} 3 \mathrm{C}$ & $1.384(6)$ \\
\hline $\mathrm{O} 2 \mathrm{~A}-\mathrm{C} 5 \mathrm{~B}$ & $1.410(5)$ & $\mathrm{C} 2 \mathrm{C}-\mathrm{C} 8 \mathrm{C}$ & $1.529(6)$ \\
\hline $\mathrm{P} 1 \mathrm{~B}-\mathrm{O} 3 \mathrm{~B}$ & $1.465(3)$ & $\mathrm{C} 3 \mathrm{C}-\mathrm{C} 4 \mathrm{C}$ & $1.392(6)$ \\
\hline $\mathrm{P} 1 \mathrm{~B}-\mathrm{O} 1 \mathrm{~B}$ & $1.582(3)$ & $\mathrm{C} 4 \mathrm{C}-\mathrm{C} 5 \mathrm{C}$ & $1.392(6)$ \\
\hline $\mathrm{P} 1 \mathrm{~B}-\mathrm{O} 2 \mathrm{~B}$ & $1.598(3)$ & $\mathrm{C} 4 \mathrm{C}-\mathrm{C} 7 \mathrm{C}$ & $1.506(6)$ \\
\hline $\mathrm{P} 1 \mathrm{~B}-\mathrm{C} 12 \mathrm{~B}$ & $1.771(5)$ & $\mathrm{C} 5 \mathrm{C}-\mathrm{C} 6 \mathrm{C}$ & $1.391(6)$ \\
\hline $\mathrm{O} 1 \mathrm{~B}-\mathrm{C} 3 \mathrm{~B}$ & $1.409(5)$ & $\mathrm{C} 7 \mathrm{C}-\mathrm{H} 7 \mathrm{C} 1$ & 0.9800 \\
\hline $\mathrm{O} 2 \mathrm{~B}-\mathrm{C} 5 \mathrm{C}$ & $1.415(5)$ & $\mathrm{C} 7 \mathrm{C}-\mathrm{H} 7 \mathrm{C} 2$ & 0.9800 \\
\hline $\mathrm{P} 1 \mathrm{C}-\mathrm{O} 3 \mathrm{C}$ & $1.465(3)$ & $\mathrm{C} 7 \mathrm{C}-\mathrm{H} 7 \mathrm{C} 3$ & 0.9800 \\
\hline $\mathrm{P} 1 \mathrm{C}-\mathrm{O} 1 \mathrm{C}$ & $1.584(3)$ & $\mathrm{C} 8 \mathrm{C}-\mathrm{C} 6 \mathrm{D}$ & $1.525(5)$ \\
\hline $\mathrm{P} 1 \mathrm{C}-\mathrm{O} 2 \mathrm{C}$ & $1.592(3)$ & $\mathrm{C} 8 \mathrm{C}-\mathrm{C} 9 \mathrm{C}$ & $1.528(6)$ \\
\hline $\mathrm{P} 1 \mathrm{C}-\mathrm{C} 12 \mathrm{C}$ & $1.779(5)$ & $\mathrm{C} 8 \mathrm{C}-\mathrm{H} 8 \mathrm{C}$ & 1.0000 \\
\hline $\mathrm{O} 1 \mathrm{C}-\mathrm{C} 3 \mathrm{C}$ & $1.419(5)$ & $\mathrm{C} 9 \mathrm{C}-\mathrm{C} 10 \mathrm{C}$ & $1.501(6)$ \\
\hline $\mathrm{O} 2 \mathrm{C}-\mathrm{C} 5 \mathrm{D}$ & $1.418(5)$ & $\mathrm{C} 9 \mathrm{C}-\mathrm{H} 9 \mathrm{C} 1$ & 0.9900 \\
\hline $\mathrm{P} 1 \mathrm{D}-\mathrm{O} 3 \mathrm{D}$ & $1.463(3)$ & $\mathrm{C} 9 \mathrm{C}-\mathrm{H} 9 \mathrm{C} 2$ & 0.9900 \\
\hline P1D-O1D & $1.590(3)$ & $\mathrm{C} 10 \mathrm{C}-\mathrm{C} 11 \mathrm{C}$ & $1.533(7)$ \\
\hline $\mathrm{P} 1 \mathrm{D}-\mathrm{O} 2 \mathrm{D}$ & $1.592(3)$ & $\mathrm{C} 10 \mathrm{C}-\mathrm{H} 10 \mathrm{H}$ & 0.9900 \\
\hline $\mathrm{P} 1 \mathrm{D}-\mathrm{C} 12 \mathrm{D}$ & $1.781(4)$ & $\mathrm{C} 10 \mathrm{C}-\mathrm{H} 10 \mathrm{G}$ & 0.9900 \\
\hline $\mathrm{O} 1 \mathrm{D}-\mathrm{C} 3 \mathrm{D}$ & $1.416(5)$ & $\mathrm{C} 11 \mathrm{C}-\mathrm{H} 11 \mathrm{O}$ & 0.9800 \\
\hline $\mathrm{O} 2 \mathrm{D}-\mathrm{C} 5 \mathrm{~A}$ & $1.418(5)$ & $\mathrm{C} 11 \mathrm{C}-\mathrm{H} 11 \mathrm{P}$ & 0.9800 \\
\hline $\mathrm{C} 10 \mathrm{D}-\mathrm{C} 11 \mathrm{D}$ & $1.515(7)$ & $\mathrm{C} 11 \mathrm{C}-\mathrm{H} 11 \mathrm{Q}$ & 0.9800 \\
\hline $\mathrm{C} 10 \mathrm{D}-\mathrm{C} 9 \mathrm{D}$ & $1.534(6)$ & $\mathrm{C} 12 \mathrm{C}-\mathrm{C} 13 \mathrm{C}$ & $1.384(8)$ \\
\hline $\mathrm{C} 10 \mathrm{D}-\mathrm{H} 10 \mathrm{~K}$ & 0.9900 & $\mathrm{C} 12 \mathrm{C}-\mathrm{C} 17 \mathrm{C}$ & $1.389(7)$ \\
\hline $\mathrm{C} 10 \mathrm{D}-\mathrm{H} 10 \mathrm{~J}$ & 0.9900 & $\mathrm{C} 13 \mathrm{C}-\mathrm{C} 14 \mathrm{C}$ & $1.413(9)$ \\
\hline $\mathrm{C} 2 \mathrm{~A}-\mathrm{C} 1 \mathrm{~A}$ & $1.393(6)$ & $\mathrm{C} 13 \mathrm{C}-\mathrm{H} 13 \mathrm{C}$ & 0.9500 \\
\hline $\mathrm{C} 2 \mathrm{~A}-\mathrm{C} 3 \mathrm{~A}$ & $1.396(6)$ & $\mathrm{C} 14 \mathrm{C}-\mathrm{C} 15 \mathrm{C}$ & $1.352(9)$ \\
\hline $\mathrm{C} 2 \mathrm{~A}-\mathrm{C} 8 \mathrm{~A}$ & $1.529(6)$ & $\mathrm{C} 14 \mathrm{C}-\mathrm{H} 14 \mathrm{C}$ & 0.9500 \\
\hline $\mathrm{C} 3 \mathrm{~A}-\mathrm{C} 4 \mathrm{~A}$ & $1.375(6)$ & $\mathrm{C} 15 \mathrm{C}-\mathrm{C} 16 \mathrm{C}$ & $1.340(9)$ \\
\hline $\mathrm{C} 4 \mathrm{~A}-\mathrm{C} 5 \mathrm{~A}$ & $1.398(5)$ & $\mathrm{C} 15 \mathrm{C}-\mathrm{H} 15 \mathrm{C}$ & 0.9500 \\
\hline $\mathrm{C} 4 \mathrm{~A}-\mathrm{C} 7 \mathrm{~A}$ & $1.500(6)$ & $\mathrm{C} 16 \mathrm{C}-\mathrm{C} 17 \mathrm{C}$ & $1.389(7)$ \\
\hline $\mathrm{C} 5 \mathrm{~A}-\mathrm{C} 6 \mathrm{~A}$ & $1.394(5)$ & $\mathrm{C} 16 \mathrm{C}-\mathrm{H} 16 \mathrm{C}$ & 0.9500 \\
\hline
\end{tabular}




\begin{tabular}{|c|c|c|c|}
\hline $\mathrm{C} 6 \mathrm{~A}-\mathrm{C} 1 \mathrm{~A}$ & $1.391(6)$ & $\mathrm{C} 17 \mathrm{C}-\mathrm{H} 17 \mathrm{C}$ & 0.9500 \\
\hline $\mathrm{C} 6 \mathrm{~A}-\mathrm{C} 8 \mathrm{D}$ & $1.518(6)$ & $\mathrm{C} 1 \mathrm{D}-\mathrm{C} 2 \mathrm{D}$ & $1.395(5)$ \\
\hline $\mathrm{C} 7 \mathrm{~A}-\mathrm{H} 7 \mathrm{~A} 1$ & 0.9800 & $\mathrm{C} 1 \mathrm{D}-\mathrm{C} 6 \mathrm{D}$ & $1.406(6)$ \\
\hline $\mathrm{C} 7 \mathrm{~A}-\mathrm{H} 7 \mathrm{~A} 2$ & 0.9800 & $\mathrm{C} 1 \mathrm{D}-\mathrm{H} 1 \mathrm{D} 1$ & 0.9500 \\
\hline $\mathrm{C} 7 \mathrm{~A}-\mathrm{H} 7 \mathrm{~A} 3$ & 0.9800 & $\mathrm{C} 2 \mathrm{D}-\mathrm{C} 3 \mathrm{D}$ & $1.392(6)$ \\
\hline $\mathrm{C} 8 \mathrm{~A}-\mathrm{C} 9 \mathrm{E}$ & $1.524(6)$ & $\mathrm{C} 2 \mathrm{D}-\mathrm{C} 8 \mathrm{D}$ & $1.524(6)$ \\
\hline $\mathrm{C} 8 \mathrm{~A}-\mathrm{C} 9 \mathrm{~A}$ & $1.524(6)$ & $\mathrm{C} 3 \mathrm{D}-\mathrm{C} 4 \mathrm{D}$ & $1.387(6)$ \\
\hline $\mathrm{C} 8 \mathrm{~A}-\mathrm{C} 6 \mathrm{~B}$ & $1.535(6)$ & $C 4 D-C 5 D$ & $1.391(6)$ \\
\hline $\mathrm{C} 8 \mathrm{~A}-\mathrm{H} 8 \mathrm{~A}$ & 1.0000 & $\mathrm{C} 4 \mathrm{D}-\mathrm{C} 7 \mathrm{D}$ & $1.497(6)$ \\
\hline $\mathrm{C} 9 \mathrm{~A}-\mathrm{C} 10 \mathrm{~A}$ & $1.413(10)$ & $\mathrm{C} 5 \mathrm{D}-\mathrm{C} 6 \mathrm{D}$ & $1.388(6)$ \\
\hline C9A-H9A1 & 0.9900 & $\mathrm{C} 7 \mathrm{D}-\mathrm{H} 7 \mathrm{D} 1$ & 0.9800 \\
\hline C9A-H9A2 & 0.9900 & $\mathrm{C} 7 \mathrm{D}-\mathrm{H} 7 \mathrm{D} 2$ & 0.9800 \\
\hline $\mathrm{C} 10 \mathrm{~A}-\mathrm{C} 11 \mathrm{~A}$ & $1.483(15)$ & $\mathrm{C} 7 \mathrm{D}-\mathrm{H} 7 \mathrm{D} 3$ & 0.9800 \\
\hline $\mathrm{C} 10 \mathrm{~A}-\mathrm{H} 10 \mathrm{~A}$ & 0.9900 & $\mathrm{C} 8 \mathrm{D}-\mathrm{C} 9 \mathrm{D}$ & $1.545(6)$ \\
\hline $\mathrm{C} 10 \mathrm{~A}-\mathrm{H} 10 \mathrm{~B}$ & 0.9900 & $\mathrm{C} 8 \mathrm{D}-\mathrm{H} 8 \mathrm{D}$ & 1.0000 \\
\hline $\mathrm{C} 11 \mathrm{~A}-\mathrm{H} 11 \mathrm{D}$ & 0.9800 & $\mathrm{C} 9 \mathrm{D}-\mathrm{H} 9 \mathrm{D} 1$ & 0.9900 \\
\hline $\mathrm{C} 11 \mathrm{~A}-\mathrm{H} 11 \mathrm{E}$ & 0.9800 & $\mathrm{C} 9 \mathrm{D}-\mathrm{H} 9 \mathrm{D} 2$ & 0.9900 \\
\hline $\mathrm{C} 11 \mathrm{~A}-\mathrm{H} 11 \mathrm{~F}$ & 0.9800 & $\mathrm{C} 1 \mathrm{~A}-\mathrm{H} 1 \mathrm{~A} 1$ & 0.9500 \\
\hline $\mathrm{C} 9 \mathrm{E}-\mathrm{C} 10 \mathrm{E}$ & $1.379(11)$ & C11D-H11R & 0.9800 \\
\hline C9E-H9E1 & 0.9900 & C11D_H11S & 0.9800 \\
\hline C9E-H9E2 & 0.9900 & $\mathrm{C} 11 \mathrm{D}-\mathrm{H} 11 \mathrm{~T}$ & 0.9800 \\
\hline $\mathrm{C} 10 \mathrm{E}-\mathrm{C} 11 \mathrm{E}$ & $1.531(17)$ & $\mathrm{C} 12 \mathrm{D}-\mathrm{C} 17 \mathrm{D}$ & $1.351(6)$ \\
\hline $\mathrm{C} 10 \mathrm{E}-\mathrm{H} 10 \mathrm{C}$ & 0.9900 & $\mathrm{C} 12 \mathrm{D}-\mathrm{C} 13 \mathrm{D}$ & $1.387(7)$ \\
\hline $\mathrm{C} 10 \mathrm{E}-\mathrm{H} 10 \mathrm{D}$ & 0.9900 & $\mathrm{C} 13 \mathrm{D}-\mathrm{C} 14 \mathrm{D}$ & $1.351(8)$ \\
\hline $\mathrm{C} 11 \mathrm{E}-\mathrm{H} 11 \mathrm{G}$ & 0.9800 & $\mathrm{C} 13 \mathrm{D}-\mathrm{H} 13 \mathrm{D}$ & 0.9500 \\
\hline $\mathrm{C} 11 \mathrm{E}-\mathrm{H} 11 \mathrm{H}$ & 0.9800 & $\mathrm{C} 14 \mathrm{D}-\mathrm{C} 15 \mathrm{D}$ & $1.367(9)$ \\
\hline C11E-H11I & 0.9800 & $\mathrm{C} 14 \mathrm{D}-\mathrm{H} 14 \mathrm{D}$ & 0.9500 \\
\hline $\mathrm{C} 12 \mathrm{~A}-\mathrm{C} 17 \mathrm{~A}$ & $1.371(7)$ & $\mathrm{C} 15 \mathrm{D}-\mathrm{C} 16 \mathrm{D}$ & $1.333(8)$ \\
\hline $\mathrm{C} 12 \mathrm{~A}-\mathrm{C} 13 \mathrm{~A}$ & $1.376(7)$ & $\mathrm{C} 15 \mathrm{D}-\mathrm{H} 15 \mathrm{D}$ & 0.9500 \\
\hline $\mathrm{C} 13 \mathrm{~A}-\mathrm{C} 14 \mathrm{~A}$ & $1.386(8)$ & $\mathrm{C} 16 \mathrm{D}-\mathrm{C} 17 \mathrm{D}$ & $1.389(7)$ \\
\hline $\mathrm{C} 13 \mathrm{~A}-\mathrm{H} 13 \mathrm{~A}$ & 0.9500 & $\mathrm{C} 16 \mathrm{D}-\mathrm{H} 16 \mathrm{D}$ & 0.9500 \\
\hline $\mathrm{C} 14 \mathrm{~A}-\mathrm{C} 15 \mathrm{~A}$ & $1.369(9)$ & $\mathrm{C} 17 \mathrm{D}-\mathrm{H} 17 \mathrm{D}$ & 0.9500 \\
\hline $\mathrm{C} 14 \mathrm{~A}-\mathrm{H} 14 \mathrm{~A}$ & 0.9500 & $\mathrm{O} 1 \mathrm{~S}-\mathrm{C} 1 \mathrm{~S}$ & $1.449(5)$ \\
\hline $\mathrm{C} 15 \mathrm{~A}-\mathrm{C} 16 \mathrm{~A}$ & $1.361(9)$ & $\mathrm{O} 1 \mathrm{~S}-\mathrm{H} 1 \mathrm{~S}$ & 0.8400 \\
\hline $\mathrm{C} 15 \mathrm{~A}-\mathrm{H} 15 \mathrm{~A}$ & 0.9500 & $\mathrm{C} 1 \mathrm{~S}-\mathrm{H} 1 \mathrm{~S} 1$ & 0.9800 \\
\hline $\mathrm{C} 16 \mathrm{~A}-\mathrm{C} 17 \mathrm{~A}$ & $1.387(7)$ & $\mathrm{C} 1 \mathrm{~S}-\mathrm{H} 1 \mathrm{~S} 2$ & 0.9800 \\
\hline $\mathrm{C} 16 \mathrm{~A}-\mathrm{H} 16 \mathrm{~A}$ & 0.9500 & $\mathrm{C} 1 \mathrm{~S}-\mathrm{H} 1 \mathrm{~S} 3$ & 0.9800 \\
\hline C17A-H17A & 0.9500 & $\mathrm{O} 2 \mathrm{~S}-\mathrm{C} 2 \mathrm{~S}$ & $1.425(5)$ \\
\hline $\mathrm{C} 1 \mathrm{~B}-\mathrm{C} 6 \mathrm{~B}$ & $1.391(6)$ & $\mathrm{O} 2 \mathrm{~S}-\mathrm{H} 2 \mathrm{~S}$ & 0.8400 \\
\hline $\mathrm{C} 1 \mathrm{~B}-\mathrm{C} 2 \mathrm{~B}$ & $1.403(6)$ & $\mathrm{C} 2 \mathrm{~S}-\mathrm{H} 2 \mathrm{~S} 1$ & 0.9800 \\
\hline $\mathrm{C} 1 \mathrm{~B}-\mathrm{H} 1 \mathrm{~B} 1$ & 0.9500 & $\mathrm{C} 2 \mathrm{~S}-\mathrm{H} 2 \mathrm{~S} 2$ & 0.9800 \\
\hline $\mathrm{C} 2 \mathrm{~B}-\mathrm{C} 3 \mathrm{~B}$ & $1.387(6)$ & $\mathrm{C} 2 \mathrm{~S}-\mathrm{H} 2 \mathrm{~S} 3$ & 0.9800 \\
\hline $\mathrm{C} 2 \mathrm{~B}-\mathrm{C} 8 \mathrm{~B}$ & $1.508(6)$ & & \\
\hline $\mathrm{C} 1-\mathrm{N} 1-\mathrm{C} 2$ & $113.1(4)$ & $\mathrm{C} 4 \mathrm{~B}-\mathrm{C} 7 \mathrm{~B}-\mathrm{H} 7 \mathrm{~B} 2$ & 109.5 \\
\hline $\mathrm{C} 1-\mathrm{N} 1-\mathrm{H} 1 \mathrm{~A}$ & 109.0 & $\mathrm{H} 7 \mathrm{~B} 1-\mathrm{C} 7 \mathrm{~B}-\mathrm{H} 7 \mathrm{~B} 2$ & 109.5 \\
\hline $\mathrm{C} 2-\mathrm{N} 1-\mathrm{H} 1 \mathrm{~A}$ & 109.0 & $\mathrm{C} 4 \mathrm{~B}-\mathrm{C} 7 \mathrm{~B}-\mathrm{H} 7 \mathrm{~B} 3$ & 109.5 \\
\hline $\mathrm{C} 1-\mathrm{N} 1-\mathrm{H} 1 \mathrm{~B}$ & 109.0 & $\mathrm{H} 7 \mathrm{~B} 1-\mathrm{C} 7 \mathrm{~B}-\mathrm{H} 7 \mathrm{~B} 3$ & 109.5 \\
\hline
\end{tabular}




\begin{tabular}{|c|c|c|c|}
\hline $\mathrm{C} 2-\mathrm{N} 1-\mathrm{H} 1 \mathrm{~B}$ & 109.0 & $\mathrm{H} 7 \mathrm{~B} 2-\mathrm{C} 7 \mathrm{~B}-\mathrm{H} 7 \mathrm{~B} 3$ & 109.5 \\
\hline $\mathrm{H} 1 \mathrm{~A}-\mathrm{N} 1-\mathrm{H} 1 \mathrm{~B}$ & 107.8 & $\mathrm{C} 2 \mathrm{~B}-\mathrm{C} 8 \mathrm{~B}-\mathrm{C} 9 \mathrm{~B}$ & $113.9(4)$ \\
\hline $\mathrm{N} 1-\mathrm{C} 1-\mathrm{H} 1 \mathrm{C}$ & 109.5 & $\mathrm{C} 2 \mathrm{~B}-\mathrm{C} 8 \mathrm{~B}-\mathrm{C} 6 \mathrm{C}$ & $109.0(3)$ \\
\hline $\mathrm{N} 1-\mathrm{C} 1-\mathrm{H} 1 \mathrm{D}$ & 109.5 & $\mathrm{C} 9 \mathrm{~B}-\mathrm{C} 8 \mathrm{~B}-\mathrm{C} 6 \mathrm{C}$ & $112.2(3)$ \\
\hline $\mathrm{H} 1 \mathrm{C}-\mathrm{C} 1-\mathrm{H} 1 \mathrm{D}$ & 109.5 & $\mathrm{C} 2 \mathrm{~B}-\mathrm{C} 8 \mathrm{~B}-\mathrm{H} 8 \mathrm{~B}$ & 107.1 \\
\hline $\mathrm{N} 1-\mathrm{C} 1-\mathrm{H} 1 \mathrm{E}$ & 109.5 & $\mathrm{C} 9 \mathrm{~B}-\mathrm{C} 8 \mathrm{~B}-\mathrm{H} 8 \mathrm{~B}$ & 107.1 \\
\hline $\mathrm{H} 1 \mathrm{C}-\mathrm{C} 1-\mathrm{H} 1 \mathrm{E}$ & 109.5 & $\mathrm{C} 6 \mathrm{C}-\mathrm{C} 8 \mathrm{~B}-\mathrm{H} 8 \mathrm{~B}$ & 107.1 \\
\hline $\mathrm{H} 1 \mathrm{D}-\mathrm{C} 1-\mathrm{H} 1 \mathrm{E}$ & 109.5 & $\mathrm{C} 10 \mathrm{~B}-\mathrm{C} 9 \mathrm{~B}-\mathrm{C} 8 \mathrm{~B}$ & $113.9(4)$ \\
\hline $\mathrm{C} 3-\mathrm{C} 2-\mathrm{N} 1$ & $111.6(5)$ & $\mathrm{C} 10 \mathrm{~B}-\mathrm{C} 9 \mathrm{~B}-\mathrm{H} 9 \mathrm{~B} 1$ & 108.8 \\
\hline $\mathrm{C} 3-\mathrm{C} 2-\mathrm{C} 4$ & $111.3(5)$ & $\mathrm{C} 8 \mathrm{~B}-\mathrm{C} 9 \mathrm{~B}-\mathrm{H} 9 \mathrm{~B} 1$ & 108.8 \\
\hline $\mathrm{N} 1-\mathrm{C} 2-\mathrm{C} 4$ & $108.6(4)$ & $\mathrm{C} 10 \mathrm{~B}-\mathrm{C} 9 \mathrm{~B}-\mathrm{H} 9 \mathrm{~B} 2$ & 108.8 \\
\hline $\mathrm{C} 3-\mathrm{C} 2-\mathrm{H} 2$ & 108.4 & $\mathrm{C} 8 \mathrm{~B}-\mathrm{C} 9 \mathrm{~B}-\mathrm{H} 9 \mathrm{~B} 2$ & 108.8 \\
\hline $\mathrm{N} 1-\mathrm{C} 2-\mathrm{H} 2$ & 108.4 & H9B $1-\mathrm{C} 9 \mathrm{~B}-\mathrm{H} 9 \mathrm{~B} 2$ & 107.7 \\
\hline $\mathrm{C} 4-\mathrm{C} 2-\mathrm{H} 2$ & 108.4 & $\mathrm{C} 9 \mathrm{~B}-\mathrm{C} 10 \mathrm{~B}-\mathrm{C} 11 \mathrm{~B}$ & $110.1(5)$ \\
\hline $\mathrm{C} 2-\mathrm{C} 3-\mathrm{H} 3 \mathrm{~A}$ & 109.5 & $\mathrm{C} 9 \mathrm{~B}-\mathrm{C} 10 \mathrm{~B}-\mathrm{H} 10 \mathrm{E}$ & 109.6 \\
\hline $\mathrm{C} 2-\mathrm{C} 3-\mathrm{H} 3 \mathrm{~B}$ & 109.5 & $\mathrm{C} 11 \mathrm{~B}-\mathrm{C} 10 \mathrm{~B}-\mathrm{H} 10 \mathrm{E}$ & 109.6 \\
\hline $\mathrm{H} 3 \mathrm{~A}-\mathrm{C} 3-\mathrm{H} 3 \mathrm{~B}$ & 109.5 & $\mathrm{C} 9 \mathrm{~B}-\mathrm{C} 10 \mathrm{~B}-\mathrm{H} 10 \mathrm{~F}$ & 109.6 \\
\hline $\mathrm{C} 2-\mathrm{C} 3-\mathrm{H} 3 \mathrm{C}$ & 109.5 & $\mathrm{C} 11 \mathrm{~B}-\mathrm{C} 10 \mathrm{~B}-\mathrm{H} 10 \mathrm{~F}$ & 109.6 \\
\hline $\mathrm{H} 3 \mathrm{~A}-\mathrm{C} 3-\mathrm{H} 3 \mathrm{C}$ & 109.5 & $\mathrm{H} 10 \mathrm{E}-\mathrm{C} 10 \mathrm{~B}-\mathrm{H} 10 \mathrm{~F}$ & 108.2 \\
\hline $\mathrm{H} 3 \mathrm{~B}-\mathrm{C} 3-\mathrm{H} 3 \mathrm{C}$ & 109.5 & $\mathrm{C} 10 \mathrm{~B}-\mathrm{C} 11 \mathrm{~B}-\mathrm{H} 11 \mathrm{~L}$ & 109.5 \\
\hline $\mathrm{O} 1-\mathrm{C} 4-\mathrm{C} 5$ & $122.5(5)$ & $\mathrm{C} 10 \mathrm{~B}-\mathrm{C} 11 \mathrm{~B}-\mathrm{H} 11 \mathrm{M}$ & 109.5 \\
\hline $\mathrm{O} 1-\mathrm{C} 4-\mathrm{C} 2$ & $117.7(5)$ & $\mathrm{H} 11 \mathrm{~L}-\mathrm{C} 11 \mathrm{~B}-\mathrm{H} 11 \mathrm{M}$ & 109.5 \\
\hline $\mathrm{C} 5-\mathrm{C} 4-\mathrm{C} 2$ & $119.7(4)$ & $\mathrm{C} 10 \mathrm{~B}-\mathrm{C} 11 \mathrm{~B}-\mathrm{H} 11 \mathrm{~N}$ & 109.5 \\
\hline $\mathrm{C} 10-\mathrm{C} 5-\mathrm{C} 6$ & $117.9(5)$ & $\mathrm{H} 11 \mathrm{~L}-\mathrm{C} 11 \mathrm{~B}-\mathrm{H} 11 \mathrm{~N}$ & 109.5 \\
\hline $\mathrm{C} 10-\mathrm{C} 5-\mathrm{C} 4$ & $119.5(5)$ & $\mathrm{H} 11 \mathrm{M}-\mathrm{C} 11 \mathrm{~B}-\mathrm{H} 11 \mathrm{~N}$ & 109.5 \\
\hline $\mathrm{C} 6-\mathrm{C} 5-\mathrm{C} 4$ & $122.6(5)$ & $\mathrm{C} 17 \mathrm{~B}-\mathrm{C} 12 \mathrm{~B}-\mathrm{C} 13 \mathrm{~B}$ & $119.5(4)$ \\
\hline $\mathrm{C} 7-\mathrm{C} 6-\mathrm{C} 5$ & $120.1(5)$ & $\mathrm{C} 17 \mathrm{~B}-\mathrm{C} 12 \mathrm{~B}-\mathrm{P} 1 \mathrm{~B}$ & $118.3(4)$ \\
\hline $\mathrm{C} 7-\mathrm{C} 6-\mathrm{H} 6$ & 120.0 & $\mathrm{C} 13 \mathrm{~B}-\mathrm{C} 12 \mathrm{~B}-\mathrm{P} 1 \mathrm{~B}$ & $122.2(4)$ \\
\hline $\mathrm{C} 5-\mathrm{C} 6-\mathrm{H} 6$ & 120.0 & $\mathrm{C} 12 \mathrm{~B}-\mathrm{C} 13 \mathrm{~B}-\mathrm{C} 14 \mathrm{~B}$ & $120.1(5)$ \\
\hline $\mathrm{C} 6-\mathrm{C} 7-\mathrm{C} 8$ & $121.8(6)$ & $\mathrm{C} 12 \mathrm{~B}-\mathrm{C} 13 \mathrm{~B}-\mathrm{H} 13 \mathrm{~B}$ & 119.9 \\
\hline $\mathrm{C} 6-\mathrm{C} 7-\mathrm{H} 7$ & 119.1 & $\mathrm{C} 14 \mathrm{~B}-\mathrm{C} 13 \mathrm{~B}-\mathrm{H} 13 \mathrm{~B}$ & 119.9 \\
\hline $\mathrm{C} 8-\mathrm{C} 7-\mathrm{H} 7$ & 119.1 & $\mathrm{C} 15 \mathrm{~B}-\mathrm{C} 14 \mathrm{~B}-\mathrm{C} 13 \mathrm{~B}$ & $119.6(5)$ \\
\hline $\mathrm{C} 9-\mathrm{C} 8-\mathrm{C} 7$ & $117.4(6)$ & $\mathrm{C} 15 \mathrm{~B}-\mathrm{C} 14 \mathrm{~B}-\mathrm{H} 14 \mathrm{~B}$ & 120.2 \\
\hline $\mathrm{C} 9-\mathrm{C} 8-\mathrm{C} 11$ & $120.3(5)$ & $\mathrm{C} 13 \mathrm{~B}-\mathrm{C} 14 \mathrm{~B}-\mathrm{H} 14 \mathrm{~B}$ & 120.2 \\
\hline $\mathrm{C} 7-\mathrm{C} 8-\mathrm{C} 11$ & $122.3(6)$ & $\mathrm{C} 16 \mathrm{~B}-\mathrm{C} 15 \mathrm{~B}-\mathrm{C} 14 \mathrm{~B}$ & $121.3(5)$ \\
\hline $\mathrm{C} 10-\mathrm{C} 9-\mathrm{C} 8$ & $121.4(5)$ & $\mathrm{C} 16 \mathrm{~B}-\mathrm{C} 15 \mathrm{~B}-\mathrm{H} 15 \mathrm{~B}$ & 119.4 \\
\hline $\mathrm{C} 10-\mathrm{C} 9-\mathrm{H} 9$ & 119.3 & $\mathrm{C} 14 \mathrm{~B}-\mathrm{C} 15 \mathrm{~B}-\mathrm{H} 15 \mathrm{~B}$ & 119.4 \\
\hline $\mathrm{C} 8-\mathrm{C} 9-\mathrm{H} 9$ & 119.3 & $\mathrm{C} 15 \mathrm{~B}-\mathrm{C} 16 \mathrm{~B}-\mathrm{C} 17 \mathrm{~B}$ & $119.5(6)$ \\
\hline $\mathrm{C} 9-\mathrm{C} 10-\mathrm{C} 5$ & $121.3(6)$ & $\mathrm{C} 15 \mathrm{~B}-\mathrm{C} 16 \mathrm{~B}-\mathrm{H} 16 \mathrm{~B}$ & 120.2 \\
\hline $\mathrm{C} 9-\mathrm{C} 10-\mathrm{H} 10$ & 119.4 & $\mathrm{C} 17 \mathrm{~B}-\mathrm{C} 16 \mathrm{~B}-\mathrm{H} 16 \mathrm{~B}$ & 120.2 \\
\hline $\mathrm{C} 5-\mathrm{C} 10-\mathrm{H} 10$ & 119.4 & $\mathrm{C} 12 \mathrm{~B}-\mathrm{C} 17 \mathrm{~B}-\mathrm{C} 16 \mathrm{~B}$ & $120.0(5)$ \\
\hline $\mathrm{C} 8-\mathrm{C} 11-\mathrm{H} 11 \mathrm{~A}$ & 109.5 & $\mathrm{C} 12 \mathrm{~B}-\mathrm{C} 17 \mathrm{~B}-\mathrm{H} 17 \mathrm{~B}$ & 120.0 \\
\hline $\mathrm{C} 8-\mathrm{C} 11-\mathrm{H} 11 \mathrm{~B}$ & 109.5 & $\mathrm{C} 16 \mathrm{~B}-\mathrm{C} 17 \mathrm{~B}-\mathrm{H} 17 \mathrm{~B}$ & 120.0 \\
\hline $\mathrm{H} 11 \mathrm{~A}-\mathrm{C} 11-\mathrm{H} 11 \mathrm{~B}$ & 109.5 & $\mathrm{C} 6 \mathrm{C}-\mathrm{C} 1 \mathrm{C}-\mathrm{C} 2 \mathrm{C}$ & $122.0(4)$ \\
\hline $\mathrm{C} 8-\mathrm{C} 11-\mathrm{H} 11 \mathrm{C}$ & 109.5 & $\mathrm{C} 6 \mathrm{C}-\mathrm{C} 1 \mathrm{C}-\mathrm{H} 1 \mathrm{C} 1$ & 119.0 \\
\hline $\mathrm{H} 11 \mathrm{~A}-\mathrm{C} 11-\mathrm{H} 11 \mathrm{C}$ & 109.5 & $\mathrm{C} 2 \mathrm{C}-\mathrm{C} 1 \mathrm{C}-\mathrm{H} 1 \mathrm{C} 1$ & 119.0 \\
\hline $\mathrm{H} 11 \mathrm{~B}-\mathrm{C} 11-\mathrm{H} 11 \mathrm{C}$ & 109.5 & $\mathrm{C} 3 \mathrm{C}-\mathrm{C} 2 \mathrm{C}-\mathrm{C} 1 \mathrm{C}$ & $117.3(4)$ \\
\hline $\mathrm{O} 3 \mathrm{~A}-\mathrm{P} 1 \mathrm{~A}-\mathrm{O} 1 \mathrm{~A}$ & $114.32(17)$ & $\mathrm{C} 3 \mathrm{C}-\mathrm{C} 2 \mathrm{C}-\mathrm{C} 8 \mathrm{C}$ & $122.3(4)$ \\
\hline
\end{tabular}




\begin{tabular}{|c|c|}
\hline $\mathrm{O} 3 \mathrm{~A}-\mathrm{P} 1 \mathrm{~A}-\mathrm{O} 2 \mathrm{~A}$ & $112.78(17)$ \\
\hline $\mathrm{O} 1 \mathrm{~A}-\mathrm{P} 1 \mathrm{~A}-\mathrm{O} 2 \mathrm{~A}$ & $105.75(17)$ \\
\hline $\mathrm{O} 3 \mathrm{~A}-\mathrm{P} 1 \mathrm{~A}-\mathrm{C} 12 \mathrm{~A}$ & $117.9(2)$ \\
\hline $\mathrm{O} 1 \mathrm{~A}-\mathrm{P} 1 \mathrm{~A}-\mathrm{C} 12 \mathrm{~A}$ & $102.66(18)$ \\
\hline $\mathrm{O} 2 \mathrm{~A}-\mathrm{P} 1 \mathrm{~A}-\mathrm{C} 12 \mathrm{~A}$ & $101.89(18)$ \\
\hline $\mathrm{C} 3 \mathrm{~A}-\mathrm{O} 1 \mathrm{~A}-\mathrm{P} 1 \mathrm{~A}$ & $121.3(2)$ \\
\hline $\mathrm{C} 5 \mathrm{~B}-\mathrm{O} 2 \mathrm{~A}-\mathrm{P} 1 \mathrm{~A}$ & $120.6(3)$ \\
\hline $\mathrm{O} 3 \mathrm{~B}-\mathrm{P} 1 \mathrm{~B}-\mathrm{O} 1 \mathrm{~B}$ & $113.97(18)$ \\
\hline $\mathrm{O} 3 \mathrm{~B}-\mathrm{P} 1 \mathrm{~B}-\mathrm{O} 2 \mathrm{~B}$ & $112.80(17)$ \\
\hline $\mathrm{O} 1 \mathrm{~B}-\mathrm{P} 1 \mathrm{~B}-\mathrm{O} 2 \mathrm{~B}$ & $105.85(16)$ \\
\hline $\mathrm{O} 3 \mathrm{~B}-\mathrm{P} 1 \mathrm{~B}-\mathrm{C} 12 \mathrm{~B}$ & $117.0(2)$ \\
\hline $\mathrm{O} 1 \mathrm{~B}-\mathrm{P} 1 \mathrm{~B}-\mathrm{C} 12 \mathrm{~B}$ & $102.63(19)$ \\
\hline $\mathrm{O} 2 \mathrm{~B}-\mathrm{P} 1 \mathrm{~B}-\mathrm{C} 12 \mathrm{~B}$ & $103.30(18)$ \\
\hline $\mathrm{C} 3 \mathrm{~B}-\mathrm{O} 1 \mathrm{~B}-\mathrm{P} 1 \mathrm{~B}$ & $121.6(3)$ \\
\hline $\mathrm{C} 5 \mathrm{C}-\mathrm{O} 2 \mathrm{~B}-\mathrm{P} 1 \mathrm{~B}$ & $121.3(2)$ \\
\hline $\mathrm{O} 3 \mathrm{C}-\mathrm{P} 1 \mathrm{C}-\mathrm{O} 1 \mathrm{C}$ & $113.94(18)$ \\
\hline $\mathrm{O} 3 \mathrm{C}-\mathrm{P} 1 \mathrm{C}-\mathrm{O} 2 \mathrm{C}$ & $114.15(18)$ \\
\hline $\mathrm{O} 1 \mathrm{C}-\mathrm{P} 1 \mathrm{C}-\mathrm{O} 2 \mathrm{C}$ & $105.73(16)$ \\
\hline $\mathrm{O} 3 \mathrm{C}-\mathrm{P} 1 \mathrm{C}-\mathrm{C} 12 \mathrm{C}$ & $117.3(2)$ \\
\hline $\mathrm{O} 1 \mathrm{C}-\mathrm{P} 1 \mathrm{C}-\mathrm{C} 12 \mathrm{C}$ & $103.55(19)$ \\
\hline $\mathrm{O} 2 \mathrm{C}-\mathrm{P} 1 \mathrm{C}-\mathrm{C} 12 \mathrm{C}$ & $100.50(19)$ \\
\hline $\mathrm{C} 3 \mathrm{C}-\mathrm{O} 1 \mathrm{C}-\mathrm{P} 1 \mathrm{C}$ & $121.9(2)$ \\
\hline $\mathrm{C} 5 \mathrm{D}-\mathrm{O} 2 \mathrm{C}-\mathrm{P} 1 \mathrm{C}$ & $122.6(3)$ \\
\hline $\mathrm{O} 3 \mathrm{D}-\mathrm{P} 1 \mathrm{D}-\mathrm{O} 1 \mathrm{D}$ & $114.22(17)$ \\
\hline $\mathrm{O} 3 \mathrm{D}-\mathrm{P} 1 \mathrm{D}-\mathrm{O} 2 \mathrm{D}$ & $113.11(17)$ \\
\hline $\mathrm{O} 1 \mathrm{D}-\mathrm{P} 1 \mathrm{D}-\mathrm{O} 2 \mathrm{D}$ & $105.32(15)$ \\
\hline $\mathrm{O} 3 \mathrm{D}-\mathrm{P} 1 \mathrm{D}-\mathrm{C} 12 \mathrm{D}$ & $117.0(2)$ \\
\hline $\mathrm{O} 1 \mathrm{D}-\mathrm{P} 1 \mathrm{D}-\mathrm{C} 12 \mathrm{D}$ & $102.41(18)$ \\
\hline $\mathrm{O} 2 \mathrm{D}-\mathrm{P} 1 \mathrm{D}-\mathrm{C} 12 \mathrm{D}$ & $103.36(17)$ \\
\hline $\mathrm{C} 3 \mathrm{D}-\mathrm{O} 1 \mathrm{D}-\mathrm{P} 1 \mathrm{D}$ & $121.0(2)$ \\
\hline $\mathrm{C} 5 \mathrm{~A}-\mathrm{O} 2 \mathrm{D}-\mathrm{P} 1 \mathrm{D}$ & $120.7(2)$ \\
\hline $\mathrm{C} 11 \mathrm{D}-\mathrm{C} 10 \mathrm{D}-\mathrm{C} 9 \mathrm{D}$ & $113.0(4)$ \\
\hline $\mathrm{C} 11 \mathrm{D}-\mathrm{C} 10 \mathrm{D}-\mathrm{H} 10 \mathrm{~K}$ & 109.0 \\
\hline $\mathrm{C} 9 \mathrm{D}-\mathrm{C} 10 \mathrm{D}-\mathrm{H} 10 \mathrm{~K}$ & 109.0 \\
\hline C11D-C10D-H10J & 109.0 \\
\hline $\mathrm{C} 9 \mathrm{D}-\mathrm{C} 10 \mathrm{D}-\mathrm{H} 10 \mathrm{~J}$ & 109.0 \\
\hline $\mathrm{H} 10 \mathrm{~K}-\mathrm{C} 10 \mathrm{D}-\mathrm{H} 10 \mathrm{~J}$ & 107.8 \\
\hline $\mathrm{C} 1 \mathrm{~A}-\mathrm{C} 2 \mathrm{~A}-\mathrm{C} 3 \mathrm{~A}$ & $116.9(4)$ \\
\hline $\mathrm{C} 1 \mathrm{~A}-\mathrm{C} 2 \mathrm{~A}-\mathrm{C} 8 \mathrm{~A}$ & $121.2(4)$ \\
\hline $\mathrm{C} 3 \mathrm{~A}-\mathrm{C} 2 \mathrm{~A}-\mathrm{C} 8 \mathrm{~A}$ & $121.8(4)$ \\
\hline $\mathrm{C} 4 \mathrm{~A}-\mathrm{C} 3 \mathrm{~A}-\mathrm{C} 2 \mathrm{~A}$ & $124.4(4)$ \\
\hline $\mathrm{C} 4 \mathrm{~A}-\mathrm{C} 3 \mathrm{~A}-\mathrm{O} 1 \mathrm{~A}$ & $117.3(3)$ \\
\hline $\mathrm{C} 2 \mathrm{~A}-\mathrm{C} 3 \mathrm{~A}-\mathrm{O} 1 \mathrm{~A}$ & $118.2(4)$ \\
\hline $\mathrm{C} 3 \mathrm{~A}-\mathrm{C} 4 \mathrm{~A}-\mathrm{C} 5 \mathrm{~A}$ & $115.6(4)$ \\
\hline $\mathrm{C} 3 \mathrm{~A}-\mathrm{C} 4 \mathrm{~A}-\mathrm{C} 7 \mathrm{~A}$ & $122.4(4)$ \\
\hline $\mathrm{C} 5 \mathrm{~A}-\mathrm{C} 4 \mathrm{~A}-\mathrm{C} 7 \mathrm{~A}$ & $122.0(4)$ \\
\hline $\mathrm{C} 6 \mathrm{~A}-\mathrm{C} 5 \mathrm{~A}-\mathrm{C} 4 \mathrm{~A}$ & $123.7(4)$ \\
\hline & \\
\hline
\end{tabular}

\begin{tabular}{|c|c|}
\hline $\mathrm{C} 1 \mathrm{C}-\mathrm{C} 2 \mathrm{C}-\mathrm{C} 8 \mathrm{C}$ & $120.4(4)$ \\
\hline $\mathrm{C} 2 \mathrm{C}-\mathrm{C} 3 \mathrm{C}-\mathrm{C} 4 \mathrm{C}$ & $123.8(4)$ \\
\hline $\mathrm{C} 2 \mathrm{C}-\mathrm{C} 3 \mathrm{C}-\mathrm{O} 1 \mathrm{C}$ & $119.3(4)$ \\
\hline $\mathrm{C} 4 \mathrm{C}-\mathrm{C} 3 \mathrm{C}-\mathrm{O} 1 \mathrm{C}$ & $116.9(4)$ \\
\hline $\mathrm{C} 3 \mathrm{C}-\mathrm{C} 4 \mathrm{C}-\mathrm{C} 5 \mathrm{C}$ & $116.0(4)$ \\
\hline $\mathrm{C} 3 \mathrm{C}-\mathrm{C} 4 \mathrm{C}-\mathrm{C} 7 \mathrm{C}$ & $121.9(4)$ \\
\hline $\mathrm{C} 5 \mathrm{C}-\mathrm{C} 4 \mathrm{C}-\mathrm{C} 7 \mathrm{C}$ & $122.1(4)$ \\
\hline $\mathrm{C} 6 \mathrm{C}-\mathrm{C} 5 \mathrm{C}-\mathrm{C} 4 \mathrm{C}$ & $123.5(4)$ \\
\hline $\mathrm{C} 6 \mathrm{C}-\mathrm{C} 5 \mathrm{C}-\mathrm{O} 2 \mathrm{~B}$ & $119.1(4)$ \\
\hline $\mathrm{C} 4 \mathrm{C}-\mathrm{C} 5 \mathrm{C}-\mathrm{O} 2 \mathrm{~B}$ & $117.4(4)$ \\
\hline $\mathrm{C} 5 \mathrm{C}-\mathrm{C} 6 \mathrm{C}-\mathrm{C} 1 \mathrm{C}$ & $117.3(4)$ \\
\hline $\mathrm{C} 5 \mathrm{C}-\mathrm{C} 6 \mathrm{C}-\mathrm{C} 8 \mathrm{~B}$ & $121.9(4)$ \\
\hline $\mathrm{C} 1 \mathrm{C}-\mathrm{C} 6 \mathrm{C}-\mathrm{C} 8 \mathrm{~B}$ & $120.8(4)$ \\
\hline $\mathrm{C} 4 \mathrm{C}-\mathrm{C} 7 \mathrm{C}-\mathrm{H} 7 \mathrm{C} 1$ & 109.5 \\
\hline $\mathrm{C} 4 \mathrm{C}-\mathrm{C} 7 \mathrm{C}-\mathrm{H} 7 \mathrm{C} 2$ & 109.5 \\
\hline $\mathrm{H} 7 \mathrm{C} 1-\mathrm{C} 7 \mathrm{C}-\mathrm{H} 7 \mathrm{C} 2$ & 109.5 \\
\hline $\mathrm{C} 4 \mathrm{C}-\mathrm{C} 7 \mathrm{C}-\mathrm{H} 7 \mathrm{C} 3$ & 109.5 \\
\hline $\mathrm{H} 7 \mathrm{C} 1-\mathrm{C} 7 \mathrm{C}-\mathrm{H} 7 \mathrm{C} 3$ & 109.5 \\
\hline $\mathrm{H} 7 \mathrm{C} 2-\mathrm{C} 7 \mathrm{C}-\mathrm{H} 7 \mathrm{C} 3$ & 109.5 \\
\hline $\mathrm{C} 6 \mathrm{D}-\mathrm{C} 8 \mathrm{C}-\mathrm{C} 9 \mathrm{C}$ & $114.9(3)$ \\
\hline $\mathrm{C} 6 \mathrm{D}-\mathrm{C} 8 \mathrm{C}-\mathrm{C} 2 \mathrm{C}$ & $108.1(3)$ \\
\hline $\mathrm{C} 9 \mathrm{C}-\mathrm{C} 8 \mathrm{C}-\mathrm{C} 2 \mathrm{C}$ & $112.3(3)$ \\
\hline $\mathrm{C} 6 \mathrm{D}-\mathrm{C} 8 \mathrm{C}-\mathrm{H} 8 \mathrm{C}$ & 107.0 \\
\hline $\mathrm{C} 9 \mathrm{C}-\mathrm{C} 8 \mathrm{C}-\mathrm{H} 8 \mathrm{C}$ & 107.0 \\
\hline $\mathrm{C} 2 \mathrm{C}-\mathrm{C} 8 \mathrm{C}-\mathrm{H} 8 \mathrm{C}$ & 107.0 \\
\hline $\mathrm{C} 10 \mathrm{C}-\mathrm{C} 9 \mathrm{C}-\mathrm{C} 8 \mathrm{C}$ & $114.6(4)$ \\
\hline $\mathrm{C} 10 \mathrm{C}-\mathrm{C} 9 \mathrm{C}-\mathrm{H} 9 \mathrm{C} 1$ & 108.6 \\
\hline $\mathrm{C} 8 \mathrm{C}-\mathrm{C} 9 \mathrm{C}-\mathrm{H} 9 \mathrm{C} 1$ & 108.6 \\
\hline $\mathrm{C} 10 \mathrm{C}-\mathrm{C} 9 \mathrm{C}-\mathrm{H} 9 \mathrm{C} 2$ & 108.6 \\
\hline $\mathrm{C} 8 \mathrm{C}-\mathrm{C} 9 \mathrm{C}-\mathrm{H} 9 \mathrm{C} 2$ & 108.6 \\
\hline $\mathrm{H} 9 \mathrm{C} 1-\mathrm{C} 9 \mathrm{C}-\mathrm{H} 9 \mathrm{C} 2$ & 107.6 \\
\hline $\mathrm{C} 9 \mathrm{C}-\mathrm{C} 10 \mathrm{C}-\mathrm{C} 11 \mathrm{C}$ & $111.9(4)$ \\
\hline $\mathrm{C} 9 \mathrm{C}-\mathrm{C} 10 \mathrm{C}-\mathrm{H} 10 \mathrm{H}$ & 109.2 \\
\hline $\mathrm{C} 11 \mathrm{C}-\mathrm{C} 10 \mathrm{C}-\mathrm{H} 10 \mathrm{H}$ & 109.2 \\
\hline $\mathrm{C} 9 \mathrm{C}-\mathrm{C} 10 \mathrm{C}-\mathrm{H} 10 \mathrm{G}$ & 109.2 \\
\hline $\mathrm{C} 11 \mathrm{C}-\mathrm{C} 10 \mathrm{C}-\mathrm{H} 10 \mathrm{G}$ & 109.2 \\
\hline $\mathrm{H} 10 \mathrm{H}-\mathrm{C} 10 \mathrm{C}-\mathrm{H} 10 \mathrm{G}$ & 107.9 \\
\hline $\mathrm{C} 10 \mathrm{C}-\mathrm{C} 11 \mathrm{C}-\mathrm{H} 11 \mathrm{O}$ & 109.5 \\
\hline $\mathrm{C} 10 \mathrm{C}-\mathrm{C} 11 \mathrm{C}-\mathrm{H} 11 \mathrm{P}$ & 109.5 \\
\hline $\mathrm{H} 11 \mathrm{O}-\mathrm{C} 11 \mathrm{C}-\mathrm{H} 11 \mathrm{P}$ & 109.5 \\
\hline $\mathrm{C} 10 \mathrm{C}-\mathrm{C} 11 \mathrm{C}-\mathrm{H} 11 \mathrm{Q}$ & 109.5 \\
\hline $\mathrm{H} 11 \mathrm{O}-\mathrm{C} 11 \mathrm{C}-\mathrm{H} 11 \mathrm{Q}$ & 109.5 \\
\hline $\mathrm{H} 11 \mathrm{P}-\mathrm{C} 11 \mathrm{C}-\mathrm{H} 11 \mathrm{Q}$ & 109.5 \\
\hline $\mathrm{C} 13 \mathrm{C}-\mathrm{C} 12 \mathrm{C}-\mathrm{C} 17 \mathrm{C}$ & $119.4(5)$ \\
\hline $\mathrm{C} 13 \mathrm{C}-\mathrm{C} 12 \mathrm{C}-\mathrm{P} 1 \mathrm{C}$ & $119.6(4)$ \\
\hline $\mathrm{C} 17 \mathrm{C}-\mathrm{C} 12 \mathrm{C}-\mathrm{P} 1 \mathrm{C}$ & $120.8(4)$ \\
\hline $\mathrm{C} 12 \mathrm{C}-\mathrm{C} 13 \mathrm{C}-\mathrm{C} 14 \mathrm{C}$ & $118.7(6)$ \\
\hline $\mathrm{C} 12 \mathrm{C}-\mathrm{C} 13 \mathrm{C}-\mathrm{H} 13 \mathrm{C}$ & 120.7 \\
\hline
\end{tabular}




\begin{tabular}{|c|c|}
\hline $\mathrm{C} 4 \mathrm{~A}-\mathrm{C} 5 \mathrm{~A}-\mathrm{O} 2 \mathrm{D}$ & $117.0(3)$ \\
\hline $\mathrm{C} 1 \mathrm{~A}-\mathrm{C} 6 \mathrm{~A}-\mathrm{C} 5 \mathrm{~A}$ & $117.1(4)$ \\
\hline $\mathrm{C} 1 \mathrm{~A}-\mathrm{C} 6 \mathrm{~A}-\mathrm{C} 8 \mathrm{D}$ & $121.6(4)$ \\
\hline $\mathrm{C} 5 \mathrm{~A}-\mathrm{C} 6 \mathrm{~A}-\mathrm{C} 8 \mathrm{D}$ & $121.3(4)$ \\
\hline $\mathrm{C} 4 \mathrm{~A}-\mathrm{C} 7 \mathrm{~A}-\mathrm{H} 7 \mathrm{~A} 1$ & 109.5 \\
\hline $\mathrm{C} 4 \mathrm{~A}-\mathrm{C} 7 \mathrm{~A}-\mathrm{H} 7 \mathrm{~A} 2$ & 109.5 \\
\hline $\mathrm{H} 7 \mathrm{~A} 1-\mathrm{C} 7 \mathrm{~A}-\mathrm{H} 7 \mathrm{~A} 2$ & 109.5 \\
\hline $\mathrm{C} 4 \mathrm{~A}-\mathrm{C} 7 \mathrm{~A}-\mathrm{H} 7 \mathrm{~A} 3$ & 109.5 \\
\hline H7A1-C7A-H7A3 & 109.5 \\
\hline $\mathrm{H} 7 \mathrm{~A} 2-\mathrm{C} 7 \mathrm{~A}-\mathrm{H} 7 \mathrm{~A} 3$ & 109.5 \\
\hline $\mathrm{C} 9 \mathrm{E}-\mathrm{C} 8 \mathrm{~A}-\mathrm{C} 2 \mathrm{~A}$ & $113.2(4)$ \\
\hline $\mathrm{C} 9 \mathrm{~A}-\mathrm{C} 8 \mathrm{~A}-\mathrm{C} 2 \mathrm{~A}$ & $113.2(4)$ \\
\hline $\mathrm{C} 9 \mathrm{E}-\mathrm{C} 8 \mathrm{~A}-\mathrm{C} 6 \mathrm{~B}$ & $113.1(4)$ \\
\hline $\mathrm{C} 9 \mathrm{~A}-\mathrm{C} 8 \mathrm{~A}-\mathrm{C} 6 \mathrm{~B}$ & $113.1(4)$ \\
\hline $\mathrm{C} 2 \mathrm{~A}-\mathrm{C} 8 \mathrm{~A}-\mathrm{C} 6 \mathrm{~B}$ & $108.0(3)$ \\
\hline $\mathrm{C} 9 \mathrm{E}-\mathrm{C} 8 \mathrm{~A}-\mathrm{H} 8 \mathrm{~A}$ & 107.4 \\
\hline $\mathrm{C} 2 \mathrm{~A}-\mathrm{C} 8 \mathrm{~A}-\mathrm{H} 8 \mathrm{~A}$ & 107.4 \\
\hline $\mathrm{C} 6 \mathrm{~B}-\mathrm{C} 8 \mathrm{~A}-\mathrm{H} 8 \mathrm{~A}$ & 107.4 \\
\hline $\mathrm{C} 10 \mathrm{~A}-\mathrm{C} 9 \mathrm{~A}-\mathrm{C} 8 \mathrm{~A}$ & $121.6(6)$ \\
\hline $\mathrm{C} 10 \mathrm{~A}-\mathrm{C} 9 \mathrm{~A}-\mathrm{H} 9 \mathrm{~A} 1$ & 106.9 \\
\hline $\mathrm{C} 8 \mathrm{~A}-\mathrm{C} 9 \mathrm{~A}-\mathrm{H} 9 \mathrm{~A} 1$ & 106.9 \\
\hline $\mathrm{C} 10 \mathrm{~A}-\mathrm{C} 9 \mathrm{~A}-\mathrm{H} 9 \mathrm{~A} 2$ & 106.9 \\
\hline $\mathrm{C} 8 \mathrm{~A}-\mathrm{C} 9 \mathrm{~A}-\mathrm{H} 9 \mathrm{~A} 2$ & 106.9 \\
\hline H9A1-C9A-H9A2 & 106.7 \\
\hline $\mathrm{C} 9 \mathrm{~A}-\mathrm{C} 10 \mathrm{~A}-\mathrm{C} 11 \mathrm{~A}$ & $116.4(9)$ \\
\hline $\mathrm{C} 9 \mathrm{~A}-\mathrm{C} 10 \mathrm{~A}-\mathrm{H} 10 \mathrm{~A}$ & 108.2 \\
\hline $\mathrm{C} 11 \mathrm{~A}-\mathrm{C} 10 \mathrm{~A}-\mathrm{H} 10 \mathrm{~A}$ & 108.2 \\
\hline $\mathrm{C} 9 \mathrm{~A}-\mathrm{C} 10 \mathrm{~A}-\mathrm{H} 10 \mathrm{~B}$ & 108.2 \\
\hline $\mathrm{C} 11 \mathrm{~A}-\mathrm{C} 10 \mathrm{~A}-\mathrm{H} 10 \mathrm{~B}$ & 108.2 \\
\hline $\mathrm{H} 10 \mathrm{~A}-\mathrm{C} 10 \mathrm{~A}-\mathrm{H} 10 \mathrm{~B}$ & 107.3 \\
\hline $\mathrm{C} 10 \mathrm{~A}-\mathrm{C} 11 \mathrm{~A}-\mathrm{H} 11 \mathrm{D}$ & 109.5 \\
\hline $\mathrm{C} 10 \mathrm{~A}-\mathrm{C} 11 \mathrm{~A}-\mathrm{H} 11 \mathrm{E}$ & 109.5 \\
\hline $\mathrm{H} 11 \mathrm{D}-\mathrm{C} 11 \mathrm{~A}-\mathrm{H} 11 \mathrm{E}$ & 109.5 \\
\hline $\mathrm{C} 10 \mathrm{~A}-\mathrm{C} 11 \mathrm{~A}-\mathrm{H} 11 \mathrm{~F}$ & 109.5 \\
\hline H11D-C11A-H11F & 109.5 \\
\hline $\mathrm{H} 11 \mathrm{E}-\mathrm{C} 11 \mathrm{~A}-\mathrm{H} 11 \mathrm{~F}$ & 109.5 \\
\hline $\mathrm{C} 10 \mathrm{E}-\mathrm{C} 9 \mathrm{E}-\mathrm{C} 8 \mathrm{~A}$ & $114.8(6)$ \\
\hline $\mathrm{C} 10 \mathrm{E}-\mathrm{C} 9 \mathrm{E}-\mathrm{H} 9 \mathrm{E} 1$ & 108.6 \\
\hline $\mathrm{C} 8 \mathrm{~A}-\mathrm{C} 9 \mathrm{E}-\mathrm{H} 9 \mathrm{E} 1$ & 108.6 \\
\hline $\mathrm{C} 10 \mathrm{E}-\mathrm{C} 9 \mathrm{E}-\mathrm{H} 9 \mathrm{E} 2$ & 108.6 \\
\hline $\mathrm{C} 8 \mathrm{~A}-\mathrm{C} 9 \mathrm{E}-\mathrm{H} 9 \mathrm{E} 2$ & 108.6 \\
\hline H9E1-C9E-H9E2 & 107.5 \\
\hline $\mathrm{C} 9 \mathrm{E}-\mathrm{C} 10 \mathrm{E}-\mathrm{C} 11 \mathrm{E}$ & $110.3(9)$ \\
\hline $\mathrm{C} 9 \mathrm{E}-\mathrm{C} 10 \mathrm{E}-\mathrm{H} 10 \mathrm{C}$ & 109.6 \\
\hline $\mathrm{C} 11 \mathrm{E}-\mathrm{C} 10 \mathrm{E}-\mathrm{H} 10 \mathrm{C}$ & 109.6 \\
\hline $\mathrm{C} 9 \mathrm{E}-\mathrm{C} 10 \mathrm{E}-\mathrm{H} 10 \mathrm{D}$ & 109.6 \\
\hline $\mathrm{C} 11 \mathrm{E}-\mathrm{C} 10 \mathrm{E}-\mathrm{H} 10 \mathrm{D}$ & 109.6 \\
\hline $\mathrm{H} 10 \mathrm{C}-\mathrm{C} 10 \mathrm{E}-\mathrm{H} 10 \mathrm{D}$ & 108.1 \\
\hline
\end{tabular}

\begin{tabular}{|c|c|}
\hline $\mathrm{C} 14 \mathrm{C}-\mathrm{C} 13 \mathrm{C}-\mathrm{H} 13 \mathrm{C}$ & 120.7 \\
\hline $\mathrm{C} 15 \mathrm{C}-\mathrm{C} 14 \mathrm{C}-\mathrm{C} 13 \mathrm{C}$ & $120.4(6)$ \\
\hline $\mathrm{C} 15 \mathrm{C}-\mathrm{C} 14 \mathrm{C}-\mathrm{H} 14 \mathrm{C}$ & 119.8 \\
\hline $\mathrm{C} 13 \mathrm{C}-\mathrm{C} 14 \mathrm{C}-\mathrm{H} 14 \mathrm{C}$ & 119.8 \\
\hline $\mathrm{C} 16 \mathrm{C}-\mathrm{C} 15 \mathrm{C}-\mathrm{C} 14 \mathrm{C}$ & $121.0(6)$ \\
\hline $\mathrm{C} 16 \mathrm{C}-\mathrm{C} 15 \mathrm{C}-\mathrm{H} 15 \mathrm{C}$ & 119.5 \\
\hline $\mathrm{C} 14 \mathrm{C}-\mathrm{C} 15 \mathrm{C}-\mathrm{H} 15 \mathrm{C}$ & 119.5 \\
\hline $\mathrm{C} 15 \mathrm{C}-\mathrm{C} 16 \mathrm{C}-\mathrm{C} 17 \mathrm{C}$ & $120.6(6)$ \\
\hline $\mathrm{C} 15 \mathrm{C}-\mathrm{C} 16 \mathrm{C}-\mathrm{H} 16 \mathrm{C}$ & 119.7 \\
\hline $\mathrm{C} 17 \mathrm{C}-\mathrm{C} 16 \mathrm{C}-\mathrm{H} 16 \mathrm{C}$ & 119.7 \\
\hline $\mathrm{C} 12 \mathrm{C}-\mathrm{C} 17 \mathrm{C}-\mathrm{C} 16 \mathrm{C}$ & $119.8(6)$ \\
\hline $\mathrm{C} 12 \mathrm{C}-\mathrm{C} 17 \mathrm{C}-\mathrm{H} 17 \mathrm{C}$ & 120.1 \\
\hline $\mathrm{C} 16 \mathrm{C}-\mathrm{C} 17 \mathrm{C}-\mathrm{H} 17 \mathrm{C}$ & 120.1 \\
\hline $\mathrm{C} 2 \mathrm{D}-\mathrm{C} 1 \mathrm{D}-\mathrm{C} 6 \mathrm{D}$ & $121.2(4)$ \\
\hline $\mathrm{C} 2 \mathrm{D}-\mathrm{C} 1 \mathrm{D}-\mathrm{H} 1 \mathrm{D} 1$ & 119.4 \\
\hline $\mathrm{C} 6 \mathrm{D}-\mathrm{C} 1 \mathrm{D}-\mathrm{H} 1 \mathrm{D} 1$ & 119.4 \\
\hline $\mathrm{C} 3 \mathrm{D}-\mathrm{C} 2 \mathrm{D}-\mathrm{C} 1 \mathrm{D}$ & $117.9(4)$ \\
\hline $\mathrm{C} 3 \mathrm{D}-\mathrm{C} 2 \mathrm{D}-\mathrm{C} 8 \mathrm{D}$ & $120.1(4)$ \\
\hline $\mathrm{C} 1 \mathrm{D}-\mathrm{C} 2 \mathrm{D}-\mathrm{C} 8 \mathrm{D}$ & $122.0(4)$ \\
\hline $\mathrm{C} 4 \mathrm{D}-\mathrm{C} 3 \mathrm{D}-\mathrm{C} 2 \mathrm{D}$ & $123.8(4)$ \\
\hline $\mathrm{C} 4 \mathrm{D}-\mathrm{C} 3 \mathrm{D}-\mathrm{O} 1 \mathrm{D}$ & $116.6(4)$ \\
\hline $\mathrm{C} 2 \mathrm{D}-\mathrm{C} 3 \mathrm{D}-\mathrm{O} 1 \mathrm{D}$ & $119.5(3)$ \\
\hline $\mathrm{C} 3 \mathrm{D}-\mathrm{C} 4 \mathrm{D}-\mathrm{C} 5 \mathrm{D}$ & $115.5(4)$ \\
\hline $\mathrm{C} 3 \mathrm{D}-\mathrm{C} 4 \mathrm{D}-\mathrm{C} 7 \mathrm{D}$ & $122.6(4)$ \\
\hline $\mathrm{C} 5 \mathrm{D}-\mathrm{C} 4 \mathrm{D}-\mathrm{C} 7 \mathrm{D}$ & $121.9(4)$ \\
\hline $\mathrm{C} 6 \mathrm{D}-\mathrm{C} 5 \mathrm{D}-\mathrm{C} 4 \mathrm{D}$ & $124.5(4)$ \\
\hline $\mathrm{C} 6 \mathrm{D}-\mathrm{C} 5 \mathrm{D}-\mathrm{O} 2 \mathrm{C}$ & $118.2(4)$ \\
\hline $\mathrm{C} 4 \mathrm{D}-\mathrm{C} 5 \mathrm{D}-\mathrm{O} 2 \mathrm{C}$ & $117.3(4)$ \\
\hline $\mathrm{C} 5 \mathrm{D}-\mathrm{C} 6 \mathrm{D}-\mathrm{C} 1 \mathrm{D}$ & $117.1(4)$ \\
\hline $\mathrm{C} 5 \mathrm{D}-\mathrm{C} 6 \mathrm{D}-\mathrm{C} 8 \mathrm{C}$ & $120.8(4)$ \\
\hline $\mathrm{C} 1 \mathrm{D}-\mathrm{C} 6 \mathrm{D}-\mathrm{C} 8 \mathrm{C}$ & $122.1(4)$ \\
\hline $\mathrm{C} 4 \mathrm{D}-\mathrm{C} 7 \mathrm{D}-\mathrm{H} 7 \mathrm{D} 1$ & 109.5 \\
\hline $\mathrm{C} 4 \mathrm{D}-\mathrm{C} 7 \mathrm{D}-\mathrm{H} 7 \mathrm{D} 2$ & 109.5 \\
\hline $\mathrm{H} 7 \mathrm{D} 1-\mathrm{C} 7 \mathrm{D}-\mathrm{H} 7 \mathrm{D} 2$ & 109.5 \\
\hline $\mathrm{C} 4 \mathrm{D}-\mathrm{C} 7 \mathrm{D}-\mathrm{H} 7 \mathrm{D} 3$ & 109.5 \\
\hline H7D1-C7D-H7D3 & 109.5 \\
\hline $\mathrm{H} 7 \mathrm{D} 2-\mathrm{C} 7 \mathrm{D}-\mathrm{H} 7 \mathrm{D} 3$ & 109.5 \\
\hline $\mathrm{C} 6 \mathrm{~A}-\mathrm{C} 8 \mathrm{D}-\mathrm{C} 2 \mathrm{D}$ & $109.1(3)$ \\
\hline $\mathrm{C} 6 \mathrm{~A}-\mathrm{C} 8 \mathrm{D}-\mathrm{C} 9 \mathrm{D}$ & $114.1(4)$ \\
\hline $\mathrm{C} 2 \mathrm{D}-\mathrm{C} 8 \mathrm{D}-\mathrm{C} 9 \mathrm{D}$ & $113.1(3)$ \\
\hline $\mathrm{C} 6 \mathrm{~A}-\mathrm{C} 8 \mathrm{D}-\mathrm{H} 8 \mathrm{D}$ & 106.7 \\
\hline $\mathrm{C} 2 \mathrm{D}-\mathrm{C} 8 \mathrm{D}-\mathrm{H} 8 \mathrm{D}$ & 106.7 \\
\hline $\mathrm{C} 9 \mathrm{D}-\mathrm{C} 8 \mathrm{D}-\mathrm{H} 8 \mathrm{D}$ & 106.7 \\
\hline $\mathrm{C} 10 \mathrm{D}-\mathrm{C} 9 \mathrm{D}-\mathrm{C} 8 \mathrm{D}$ & $112.4(4)$ \\
\hline C10D-C9D-H9D1 & 109.1 \\
\hline $\mathrm{C} 8 \mathrm{D}-\mathrm{C} 9 \mathrm{D}-\mathrm{H} 9 \mathrm{D} 1$ & 109.1 \\
\hline $\mathrm{C} 10 \mathrm{D}-\mathrm{C} 9 \mathrm{D}-\mathrm{H} 9 \mathrm{D} 2$ & 109.1 \\
\hline $\mathrm{C} 8 \mathrm{D}-\mathrm{C} 9 \mathrm{D}-\mathrm{H} 9 \mathrm{D} 2$ & 109.1 \\
\hline
\end{tabular}

120.7

119.8

19.8

$21.0(6)$

119.5

119.5

$120.6(6)$

119.7

19.8 (6)

(20.

$21.2(4)$

9.4

17.9 (4)

$20.1(4)$

$22.0(4)$

$23.8(4)$

$16.6(4)$

$119.5(3)$

15.5 (4)

(4)

$124.5(4)$

$118.2(4)$

$17.3(4)$

$17.1(4)$

$20.8(4)$

$22.1(4)$

109.5

109.5

109.5

109.5

$113.1(3)$

106.7

106.7

106.7

112.4 (4)

109.1

109.1

109.1 
C10E-C11E-H11G

$\mathrm{C} 10 \mathrm{E}-\mathrm{C} 11 \mathrm{E}-\mathrm{H} 11 \mathrm{H}$

$\mathrm{H} 11 \mathrm{G}-\mathrm{C} 11 \mathrm{E}-\mathrm{H} 11 \mathrm{H}$

C10E-C11E-H11I

H11G-C11E-H11I

$\mathrm{H} 11 \mathrm{H}-\mathrm{C} 11 \mathrm{E}-\mathrm{H} 11 \mathrm{I}$

$\mathrm{C} 17 \mathrm{~A}-\mathrm{C} 12 \mathrm{~A}-\mathrm{C} 13 \mathrm{~A}$

$\mathrm{C} 17 \mathrm{~A}-\mathrm{C} 12 \mathrm{~A}-\mathrm{P} 1 \mathrm{~A}$

$\mathrm{C} 13 \mathrm{~A}-\mathrm{C} 12 \mathrm{~A}-\mathrm{P} 1 \mathrm{~A}$

$\mathrm{C} 12 \mathrm{~A}-\mathrm{C} 13 \mathrm{~A}-\mathrm{C} 14 \mathrm{~A}$

C12A-C13A-H13A

C14A-C13A-H13A

$\mathrm{C} 15 \mathrm{~A}-\mathrm{C} 14 \mathrm{~A}-\mathrm{C} 13 \mathrm{~A}$

C15A-C14A-H14A

C13A-C14A-H14A

C16A-C15A-C14A

C16A-C15A-H15A

C14A-C15A-H15A

$\mathrm{C} 15 \mathrm{~A}-\mathrm{C} 16 \mathrm{~A}-\mathrm{C} 17 \mathrm{~A}$

C15A-C16A-H16A

C17A-C16A-H16A

$\mathrm{C} 12 \mathrm{~A}-\mathrm{C} 17 \mathrm{~A}-\mathrm{C} 16 \mathrm{~A}$

C12A-C17A-H17A

C16A-C17A-H17A

$\mathrm{C} 6 \mathrm{~B}-\mathrm{C} 1 \mathrm{~B}-\mathrm{C} 2 \mathrm{~B}$

$\mathrm{C} 6 \mathrm{~B}-\mathrm{C} 1 \mathrm{~B}-\mathrm{H} 1 \mathrm{~B} 1$

$\mathrm{C} 2 \mathrm{~B}-\mathrm{C} 1 \mathrm{~B}-\mathrm{H} 1 \mathrm{~B} 1$

$\mathrm{C} 3 \mathrm{~B}-\mathrm{C} 2 \mathrm{~B}-\mathrm{C} 1 \mathrm{~B}$

$\mathrm{C} 3 \mathrm{~B}-\mathrm{C} 2 \mathrm{~B}-\mathrm{C} 8 \mathrm{~B}$

$\mathrm{C} 1 \mathrm{~B}-\mathrm{C} 2 \mathrm{~B}-\mathrm{C} 8 \mathrm{~B}$

$\mathrm{C} 2 \mathrm{~B}-\mathrm{C} 3 \mathrm{~B}-\mathrm{C} 4 \mathrm{~B}$

$\mathrm{C} 2 \mathrm{~B}-\mathrm{C} 3 \mathrm{~B}-\mathrm{O} 1 \mathrm{~B}$

$\mathrm{C} 4 \mathrm{~B}-\mathrm{C} 3 \mathrm{~B}-\mathrm{O} 1 \mathrm{~B}$

$\mathrm{C} 3 \mathrm{~B}-\mathrm{C} 4 \mathrm{~B}-\mathrm{C} 5 \mathrm{~B}$

$\mathrm{C} 3 \mathrm{~B}-\mathrm{C} 4 \mathrm{~B}-\mathrm{C} 7 \mathrm{~B}$

$\mathrm{C} 5 \mathrm{~B}-\mathrm{C} 4 \mathrm{~B}-\mathrm{C} 7 \mathrm{~B}$

$\mathrm{C} 6 \mathrm{~B}-\mathrm{C} 5 \mathrm{~B}-\mathrm{C} 4 \mathrm{~B}$

$\mathrm{C} 6 \mathrm{~B}-\mathrm{C} 5 \mathrm{~B}-\mathrm{O} 2 \mathrm{~A}$

$\mathrm{C} 4 \mathrm{~B}-\mathrm{C} 5 \mathrm{~B}-\mathrm{O} 2 \mathrm{~A}$

$\mathrm{C} 5 \mathrm{~B}-\mathrm{C} 6 \mathrm{~B}-\mathrm{C} 1 \mathrm{~B}$

$\mathrm{C} 5 \mathrm{~B}-\mathrm{C} 6 \mathrm{~B}-\mathrm{C} 8 \mathrm{~A}$

$\mathrm{C} 1 \mathrm{~B}-\mathrm{C} 6 \mathrm{~B}-\mathrm{C} 8 \mathrm{~A}$

$\mathrm{C} 4 \mathrm{~B}-\mathrm{C} 7 \mathrm{~B}-\mathrm{H} 7 \mathrm{~B} 1$

$\mathrm{C} 1-\mathrm{N} 1-\mathrm{C} 2-\mathrm{C} 3$

$\mathrm{C} 1-\mathrm{N} 1-\mathrm{C} 2-\mathrm{C} 4$

$\mathrm{C} 3-\mathrm{C} 2-\mathrm{C} 4-\mathrm{O} 1$

$\mathrm{N} 1-\mathrm{C} 2-\mathrm{C} 4-\mathrm{O} 1$
109.5

109.5

109.5

109.5

109.5

109.5

$119.6(4)$

$121.1(4)$

$119.2(4)$

$120.2(5)$

119.9

119.9

119.7 (6)

120.1

120.1

$120.2(5)$

119.9

119.9

120.3 (6)

119.9

119.9

120.0 (5)

120.0

120.0

121.9 (4)

119.0

119.0

$117.2(4)$

$120.9(4)$

$121.9(4)$

$124.1(4)$

$119.0(4)$

$116.9(4)$

$115.4(4)$

$121.8(4)$

$122.8(4)$

$124.0(4)$

$119.2(4)$

$116.8(4)$

$117.4(4)$

$120.5(4)$

$122.1(4)$

109.5

$67.3(6)$

$-169.7(4)$

$86.1(7)$

$-37.1(7)$
H9D1-C9D—H9D2 107.9

$\mathrm{C} 6 \mathrm{~A}-\mathrm{C} 1 \mathrm{~A}-\mathrm{C} 2 \mathrm{~A}$

$\mathrm{C} 6 \mathrm{~A}-\mathrm{C} 1 \mathrm{~A}-\mathrm{H} 1 \mathrm{~A} 1$

$\mathrm{C} 2 \mathrm{~A}-\mathrm{C} 1 \mathrm{~A}-\mathrm{H} 1 \mathrm{~A} 1$

C10D-C11D-H11R

C10D-C11D-H11S

H11R-C11D-H11S

C10D-C11D-H11T

H11R-C11D-H11T

H11S - C11D-H11T

C17D-C12D-C13D

C17D-C12D-P1D

C13D-C12D-P1D

C14D-C13D-C12D

C14D-C13D-H13D

C12D-C13D-H13D

C13D-C14D-C15D

C13D-C14D-H14D

C15D-C14D-H14D

C16D-C15D-C14D

C16D-C15D-H15D

C14D-C15D-H15D

C15D-C16D-C17D

C15D-C16D-H16D

C17D-C16D-H16D

C12D $-C 17 D-C 16 D$

C12D-C17D-H17D

C16D-C17D-H17D

C1S-O1S-H1S

O1S-C1S-H1S1

$\mathrm{O} 1 \mathrm{~S}-\mathrm{C} 1 \mathrm{~S}-\mathrm{H} 1 \mathrm{~S} 2$

$\mathrm{H} 1 \mathrm{~S} 1-\mathrm{C} 1 \mathrm{~S}-\mathrm{H} 1 \mathrm{~S} 2$

$\mathrm{O} 1 \mathrm{~S}-\mathrm{C} 1 \mathrm{~S}-\mathrm{H} 1 \mathrm{~S} 3$

H1S1-C1S-H1S3

$\mathrm{H} 1 \mathrm{~S} 2-\mathrm{C} 1 \mathrm{~S}-\mathrm{H} 1 \mathrm{~S} 3$

$\mathrm{C} 2 \mathrm{~S}-\mathrm{O} 2 \mathrm{~S}-\mathrm{H} 2 \mathrm{~S}$

$\mathrm{O} 2 \mathrm{~S}-\mathrm{C} 2 \mathrm{~S}-\mathrm{H} 2 \mathrm{~S} 1$

$\mathrm{O} 2 \mathrm{~S}-\mathrm{C} 2 \mathrm{~S}-\mathrm{H} 2 \mathrm{~S} 2$

$\mathrm{H} 2 \mathrm{~S} 1-\mathrm{C} 2 \mathrm{~S}-\mathrm{H} 2 \mathrm{~S} 2$

$\mathrm{O} 2 \mathrm{~S}-\mathrm{C} 2 \mathrm{~S}-\mathrm{H} 2 \mathrm{~S} 3$

$\mathrm{H} 2 \mathrm{~S} 1-\mathrm{C} 2 \mathrm{~S}-\mathrm{H} 2 \mathrm{~S} 3$

$\mathrm{H} 2 \mathrm{~S} 2-\mathrm{C} 2 \mathrm{~S}-\mathrm{H} 2 \mathrm{~S} 3$

122.2 (4)

118.9

118.9

109.5

109.5

109.5

109.5

109.5

109.5

$117.8(4)$

$123.8(4)$

$118.3(4)$

$120.1(5)$

119.9

119.9

$121.6(6)$

119.2

119.2

$118.6(5)$

120.7

120.7

$120.6(5)$

119.7

119.7

$121.0(5)$

119.5

119.5

109.5

109.5

109.5

109.5

109.5

109.5

109.5

109.5

109.5

109.5

109.5

109.5

109.5

109.5

$\begin{array}{ll}\mathrm{C} 2 \mathrm{~B}-\mathrm{C} 8 \mathrm{~B}-\mathrm{C} 9 \mathrm{~B}-\mathrm{C} 10 \mathrm{~B} & 56.6(5) \\ \mathrm{C} 6 \mathrm{C}-\mathrm{C} 8 \mathrm{~B}-\mathrm{C} 9 \mathrm{~B}-\mathrm{C} 10 \mathrm{~B} & -178.9(4) \\ \mathrm{C} 8 \mathrm{~B}-\mathrm{C} 9 \mathrm{~B}-\mathrm{C} 10 \mathrm{~B}-\mathrm{C} 11 \mathrm{~B} & 176.7(5) \\ \mathrm{O} 3 \mathrm{~B}-\mathrm{P} 1 \mathrm{~B}-\mathrm{C} 12 \mathrm{~B}-\mathrm{C} 17 \mathrm{~B} & 38.1(5)\end{array}$




\begin{tabular}{|c|c|}
\hline $\mathrm{C} 3-\mathrm{C} 2-\mathrm{C} 4-\mathrm{C} 5$ & $-93.1(6)$ \\
\hline $\mathrm{N} 1-\mathrm{C} 2-\mathrm{C} 4-\mathrm{C} 5$ & $143.7(5)$ \\
\hline $\mathrm{O} 1-\mathrm{C} 4-\mathrm{C} 5-\mathrm{C} 10$ & $-0.2(8)$ \\
\hline $\mathrm{C} 2-\mathrm{C} 4-\mathrm{C} 5-\mathrm{C} 10$ & $179.0(5)$ \\
\hline $\mathrm{O} 1-\mathrm{C} 4-\mathrm{C} 5-\mathrm{C} 6$ & $179.6(6)$ \\
\hline $\mathrm{C} 2-\mathrm{C} 4-\mathrm{C} 5-\mathrm{C} 6$ & $-1.2(8)$ \\
\hline $\mathrm{C} 10-\mathrm{C} 5-\mathrm{C} 6-\mathrm{C} 7$ & $2.1(9)$ \\
\hline $\mathrm{C} 4-\mathrm{C} 5-\mathrm{C} 6-\mathrm{C} 7$ & $-177.6(6)$ \\
\hline $\mathrm{C} 5-\mathrm{C} 6-\mathrm{C} 7-\mathrm{C} 8$ & $1.1(11)$ \\
\hline $\mathrm{C} 6-\mathrm{C} 7-\mathrm{C} 8-\mathrm{C} 9$ & $-3.6(11)$ \\
\hline $\mathrm{C} 6-\mathrm{C} 7-\mathrm{C} 8-\mathrm{C} 11$ & $174.1(7)$ \\
\hline $\mathrm{C} 7-\mathrm{C} 8-\mathrm{C} 9-\mathrm{C} 10$ & $2.9(10)$ \\
\hline $\mathrm{C} 11-\mathrm{C} 8-\mathrm{C} 9-\mathrm{C} 10$ & $-174.8(7)$ \\
\hline $\mathrm{C} 8-\mathrm{C} 9-\mathrm{C} 10-\mathrm{C} 5$ & $0.2(11)$ \\
\hline $\mathrm{C} 6-\mathrm{C} 5-\mathrm{C} 10-\mathrm{C} 9$ & $-2.8(9)$ \\
\hline $\mathrm{C} 4-\mathrm{C} 5-\mathrm{C} 10-\mathrm{C} 9$ & $176.9(6)$ \\
\hline $\mathrm{O} 3 \mathrm{~A}-\mathrm{P} 1 \mathrm{~A}-\mathrm{O} 1 \mathrm{~A}-\mathrm{C} 3 \mathrm{~A}$ & $-38.7(3)$ \\
\hline $\mathrm{O} 2 \mathrm{~A}-\mathrm{P} 1 \mathrm{~A}-\mathrm{O} 1 \mathrm{~A}-\mathrm{C} 3 \mathrm{~A}$ & $86.0(3)$ \\
\hline $\mathrm{C} 12 \mathrm{~A}-\mathrm{P} 1 \mathrm{~A}-\mathrm{O} 1 \mathrm{~A}-\mathrm{C} 3 \mathrm{~A}$ & $-167.6(3)$ \\
\hline $\mathrm{O} 3 \mathrm{~A}-\mathrm{P} 1 \mathrm{~A}-\mathrm{O} 2 \mathrm{~A}-\mathrm{C} 5 \mathrm{~B}$ & $38.9(4)$ \\
\hline $\mathrm{O} 1 \mathrm{~A}-\mathrm{P} 1 \mathrm{~A}-\mathrm{O} 2 \mathrm{~A}-\mathrm{C} 5 \mathrm{~B}$ & $-86.8(3)$ \\
\hline $\mathrm{C} 12 \mathrm{~A}-\mathrm{P} 1 \mathrm{~A}-\mathrm{O} 2 \mathrm{~A}-\mathrm{C} 5 \mathrm{~B}$ & $166.3(3)$ \\
\hline $\mathrm{O} 3 \mathrm{~B}-\mathrm{P} 1 \mathrm{~B}-\mathrm{O} 1 \mathrm{~B}-\mathrm{C} 3 \mathrm{~B}$ & $-36.1(4)$ \\
\hline $\mathrm{O} 2 \mathrm{~B}-\mathrm{P} 1 \mathrm{~B}-\mathrm{O} 1 \mathrm{~B}-\mathrm{C} 3 \mathrm{~B}$ & $88.4(3)$ \\
\hline $\mathrm{C} 12 \mathrm{~B}-\mathrm{P} 1 \mathrm{~B}-\mathrm{O} 1 \mathrm{~B}-\mathrm{C} 3 \mathrm{~B}$ & $-163.6(3)$ \\
\hline $\mathrm{O} 3 \mathrm{~B}-\mathrm{P} 1 \mathrm{~B}-\mathrm{O} 2 \mathrm{~B}-\mathrm{C} 5 \mathrm{C}$ & $38.8(4)$ \\
\hline $\mathrm{O} 1 \mathrm{~B}-\mathrm{P} 1 \mathrm{~B}-\mathrm{O} 2 \mathrm{~B}-\mathrm{C} 5 \mathrm{C}$ & $-86.5(3)$ \\
\hline $\mathrm{C} 12 \mathrm{~B}-\mathrm{P} 1 \mathrm{~B}-\mathrm{O} 2 \mathrm{~B}-\mathrm{C} 5 \mathrm{C}$ & $166.0(3)$ \\
\hline $\mathrm{O} 3 \mathrm{C}-\mathrm{P} 1 \mathrm{C}-\mathrm{O} 1 \mathrm{C}-\mathrm{C} 3 \mathrm{C}$ & $-44.2(4)$ \\
\hline $\mathrm{O} 2 \mathrm{C}-\mathrm{P} 1 \mathrm{C}-\mathrm{O} 1 \mathrm{C}-\mathrm{C} 3 \mathrm{C}$ & $82.0(3)$ \\
\hline $\mathrm{C} 12 \mathrm{C}-\mathrm{P} 1 \mathrm{C}-\mathrm{O} 1 \mathrm{C}-\mathrm{C} 3 \mathrm{C}$ & $-172.8(3)$ \\
\hline $\mathrm{O} 3 \mathrm{C}-\mathrm{P} 1 \mathrm{C}-\mathrm{O} 2 \mathrm{C}-\mathrm{C} 5 \mathrm{D}$ & $41.0(4)$ \\
\hline $\mathrm{O} 1 \mathrm{C}-\mathrm{P} 1 \mathrm{C}-\mathrm{O} 2 \mathrm{C}-\mathrm{C} 5 \mathrm{D}$ & $-85.0(3)$ \\
\hline $\mathrm{C} 12 \mathrm{C}-\mathrm{P} 1 \mathrm{C}-\mathrm{O} 2 \mathrm{C}-\mathrm{C} 5 \mathrm{D}$ & $167.5(3)$ \\
\hline $\mathrm{O} 3 \mathrm{D}-\mathrm{P} 1 \mathrm{D}-\mathrm{O} 1 \mathrm{D}-\mathrm{C} 3 \mathrm{D}$ & $-36.7(3)$ \\
\hline $\mathrm{O} 2 \mathrm{D}-\mathrm{P} 1 \mathrm{D}-\mathrm{O} 1 \mathrm{D}-\mathrm{C} 3 \mathrm{D}$ & $88.0(3)$ \\
\hline $\mathrm{C} 12 \mathrm{D}-\mathrm{P} 1 \mathrm{D}-\mathrm{O} 1 \mathrm{D}-\mathrm{C} 3 \mathrm{D}$ & $-164.2(3)$ \\
\hline $\mathrm{O} 3 \mathrm{D}-\mathrm{P} 1 \mathrm{D}-\mathrm{O} 2 \mathrm{D}-\mathrm{C} 5 \mathrm{~A}$ & $38.3(3)$ \\
\hline $\mathrm{O} 1 \mathrm{D}-\mathrm{P} 1 \mathrm{D}-\mathrm{O} 2 \mathrm{D}-\mathrm{C} 5 \mathrm{~A}$ & $-87.1(3)$ \\
\hline $\mathrm{C} 12 \mathrm{D}-\mathrm{P} 1 \mathrm{D}-\mathrm{O} 2 \mathrm{D}-\mathrm{C} 5 \mathrm{~A}$ & $165.8(3)$ \\
\hline $\mathrm{C} 1 \mathrm{~A}-\mathrm{C} 2 \mathrm{~A}-\mathrm{C} 3 \mathrm{~A}-\mathrm{C} 4 \mathrm{~A}$ & $0.2(6)$ \\
\hline $\mathrm{C} 8 \mathrm{~A}-\mathrm{C} 2 \mathrm{~A}-\mathrm{C} 3 \mathrm{~A}-\mathrm{C} 4 \mathrm{~A}$ & $-178.6(4)$ \\
\hline $\mathrm{C} 1 \mathrm{~A}-\mathrm{C} 2 \mathrm{~A}-\mathrm{C} 3 \mathrm{~A}-\mathrm{O} 1 \mathrm{~A}$ & $-176.6(3)$ \\
\hline $\mathrm{C} 8 \mathrm{~A}-\mathrm{C} 2 \mathrm{~A}-\mathrm{C} 3 \mathrm{~A}-\mathrm{O} 1 \mathrm{~A}$ & $4.6(6)$ \\
\hline $\mathrm{P} 1 \mathrm{~A}-\mathrm{O} 1 \mathrm{~A}-\mathrm{C} 3 \mathrm{~A}-\mathrm{C} 4 \mathrm{~A}$ & $99.2(4)$ \\
\hline $\mathrm{P} 1 \mathrm{~A}-\mathrm{O} 1 \mathrm{~A}-\mathrm{C} 3 \mathrm{~A}-\mathrm{C} 2 \mathrm{~A}$ & $-83.8(4)$ \\
\hline $\mathrm{C} 2 \mathrm{~A}-\mathrm{C} 3 \mathrm{~A}-\mathrm{C} 4 \mathrm{~A}-\mathrm{C} 5 \mathrm{~A}$ & $-1.7(6)$ \\
\hline $\mathrm{O} 1 \mathrm{~A}-\mathrm{C} 3 \mathrm{~A}-\mathrm{C} 4 \mathrm{~A}-\mathrm{C} 5 \mathrm{~A}$ & $175.1(3)$ \\
\hline
\end{tabular}

\begin{tabular}{|c|c|}
\hline $\mathrm{O} 1 \mathrm{~B}-\mathrm{P} 1 \mathrm{~B}-\mathrm{C} 12 \mathrm{~B}-\mathrm{C} 17 \mathrm{~B}$ & $163.7(4)$ \\
\hline $\mathrm{O} 2 \mathrm{~B}-\mathrm{P} 1 \mathrm{~B}-\mathrm{C} 12 \mathrm{~B}-\mathrm{C} 17 \mathrm{~B}$ & $-86.4(4)$ \\
\hline $\mathrm{O} 3 \mathrm{~B}-\mathrm{P} 1 \mathrm{~B}-\mathrm{C} 12 \mathrm{~B}-\mathrm{C} 13 \mathrm{~B}$ & $-139.4(4)$ \\
\hline $\mathrm{O} 1 \mathrm{~B}-\mathrm{P} 1 \mathrm{~B}-\mathrm{C} 12 \mathrm{~B}-\mathrm{C} 13 \mathrm{~B}$ & $-13.9(4)$ \\
\hline $\mathrm{O} 2 \mathrm{~B}-\mathrm{P} 1 \mathrm{~B}-\mathrm{C} 12 \mathrm{~B}-\mathrm{C} 13 \mathrm{~B}$ & $96.0(4)$ \\
\hline $\mathrm{C} 17 \mathrm{~B}-\mathrm{C} 12 \mathrm{~B}-\mathrm{C} 13 \mathrm{~B}-\mathrm{C} 14 \mathrm{~B}$ & $1.3(7)$ \\
\hline $\mathrm{P} 1 \mathrm{~B}-\mathrm{C} 12 \mathrm{~B}-\mathrm{C} 13 \mathrm{~B}-\mathrm{C} 14 \mathrm{~B}$ & $178.8(4)$ \\
\hline $\mathrm{C} 12 \mathrm{~B}-\mathrm{C} 13 \mathrm{~B}-\mathrm{C} 14 \mathrm{~B}-\mathrm{C} 15 \mathrm{~B}$ & $-0.7(8)$ \\
\hline $\mathrm{C} 13 \mathrm{~B}-\mathrm{C} 14 \mathrm{~B}-\mathrm{C} 15 \mathrm{~B}-\mathrm{C} 16 \mathrm{~B}$ & $-0.4(9)$ \\
\hline $\mathrm{C} 14 \mathrm{~B}-\mathrm{C} 15 \mathrm{~B}-\mathrm{C} 16 \mathrm{~B}-\mathrm{C} 17 \mathrm{~B}$ & $0.9(10)$ \\
\hline $\mathrm{C} 13 \mathrm{~B}-\mathrm{C} 12 \mathrm{~B}-\mathrm{C} 17 \mathrm{~B}-\mathrm{C} 16 \mathrm{~B}$ & $-0.8(8)$ \\
\hline $\mathrm{P} 1 \mathrm{~B}-\mathrm{C} 12 \mathrm{~B}-\mathrm{C} 17 \mathrm{~B}-\mathrm{C} 16 \mathrm{~B}$ & $-178.4(5)$ \\
\hline $\mathrm{C} 15 \mathrm{~B}-\mathrm{C} 16 \mathrm{~B}-\mathrm{C} 17 \mathrm{~B}-\mathrm{C} 12 \mathrm{~B}$ & $-0.3(9)$ \\
\hline $\mathrm{C} 6 \mathrm{C}-\mathrm{C} 1 \mathrm{C}-\mathrm{C} 2 \mathrm{C}-\mathrm{C} 3 \mathrm{C}$ & $1.8(6)$ \\
\hline $\mathrm{C} 6 \mathrm{C}-\mathrm{C} 1 \mathrm{C}-\mathrm{C} 2 \mathrm{C}-\mathrm{C} 8 \mathrm{C}$ & $-179.4(3)$ \\
\hline $\mathrm{C} 1 \mathrm{C}-\mathrm{C} 2 \mathrm{C}-\mathrm{C} 3 \mathrm{C}-\mathrm{C} 4 \mathrm{C}$ & $0.7(6)$ \\
\hline $\mathrm{C} 8 \mathrm{C}-\mathrm{C} 2 \mathrm{C}-\mathrm{C} 3 \mathrm{C}-\mathrm{C} 4 \mathrm{C}$ & $-178.0(4)$ \\
\hline $\mathrm{C} 1 \mathrm{C}-\mathrm{C} 2 \mathrm{C}-\mathrm{C} 3 \mathrm{C}-\mathrm{O} 1 \mathrm{C}$ & $-176.2(3)$ \\
\hline $\mathrm{C} 8 \mathrm{C}-\mathrm{C} 2 \mathrm{C}-\mathrm{C} 3 \mathrm{C}-\mathrm{O} 1 \mathrm{C}$ & $5.1(5)$ \\
\hline $\mathrm{P} 1 \mathrm{C}-\mathrm{O} 1 \mathrm{C}-\mathrm{C} 3 \mathrm{C}-\mathrm{C} 2 \mathrm{C}$ & $-83.3(4)$ \\
\hline $\mathrm{P} 1 \mathrm{C}-\mathrm{O} 1 \mathrm{C}-\mathrm{C} 3 \mathrm{C}-\mathrm{C} 4 \mathrm{C}$ & $99.6(4)$ \\
\hline $\mathrm{C} 2 \mathrm{C}-\mathrm{C} 3 \mathrm{C}-\mathrm{C} 4 \mathrm{C}-\mathrm{C} 5 \mathrm{C}$ & $-2.4(6)$ \\
\hline $\mathrm{O} 1 \mathrm{C}-\mathrm{C} 3 \mathrm{C}-\mathrm{C} 4 \mathrm{C}-\mathrm{C} 5 \mathrm{C}$ & $174.6(3)$ \\
\hline $\mathrm{C} 2 \mathrm{C}-\mathrm{C} 3 \mathrm{C}-\mathrm{C} 4 \mathrm{C}-\mathrm{C} 7 \mathrm{C}$ & $179.0(4)$ \\
\hline $\mathrm{O} 1 \mathrm{C}-\mathrm{C} 3 \mathrm{C}-\mathrm{C} 4 \mathrm{C}-\mathrm{C} 7 \mathrm{C}$ & $-4.0(6)$ \\
\hline $\mathrm{C} 3 \mathrm{C}-\mathrm{C} 4 \mathrm{C}-\mathrm{C} 5 \mathrm{C}-\mathrm{C} 6 \mathrm{C}$ & $1.7(6)$ \\
\hline $\mathrm{C} 7 \mathrm{C}-\mathrm{C} 4 \mathrm{C}-\mathrm{C} 5 \mathrm{C}-\mathrm{C} 6 \mathrm{C}$ & $-179.7(4)$ \\
\hline $\mathrm{C} 3 \mathrm{C}-\mathrm{C} 4 \mathrm{C}-\mathrm{C} 5 \mathrm{C}-\mathrm{O} 2 \mathrm{~B}$ & $-176.8(3)$ \\
\hline $\mathrm{C} 7 \mathrm{C}-\mathrm{C} 4 \mathrm{C}-\mathrm{C} 5 \mathrm{C}-\mathrm{O} 2 \mathrm{~B}$ & $1.8(6)$ \\
\hline $\mathrm{P} 1 \mathrm{~B}-\mathrm{O} 2 \mathrm{~B}-\mathrm{C} 5 \mathrm{C}-\mathrm{C} 6 \mathrm{C}$ & $80.1(4)$ \\
\hline $\mathrm{P} 1 \mathrm{~B}-\mathrm{O} 2 \mathrm{~B}-\mathrm{C} 5 \mathrm{C}-\mathrm{C} 4 \mathrm{C}$ & $-101.3(4)$ \\
\hline $\mathrm{C} 4 \mathrm{C}-\mathrm{C} 5 \mathrm{C}-\mathrm{C} 6 \mathrm{C}-\mathrm{C} 1 \mathrm{C}$ & $0.7(6)$ \\
\hline $\mathrm{O} 2 \mathrm{~B}-\mathrm{C} 5 \mathrm{C}-\mathrm{C} 6 \mathrm{C}-\mathrm{C} 1 \mathrm{C}$ & $179.1(3)$ \\
\hline $\mathrm{C} 4 \mathrm{C}-\mathrm{C} 5 \mathrm{C}-\mathrm{C} 6 \mathrm{C}-\mathrm{C} 8 \mathrm{~B}$ & $180.0(4)$ \\
\hline $\mathrm{O} 2 \mathrm{~B}-\mathrm{C} 5 \mathrm{C}-\mathrm{C} 6 \mathrm{C}-\mathrm{C} 8 \mathrm{~B}$ & $-1.5(5)$ \\
\hline $\mathrm{C} 2 \mathrm{C}-\mathrm{C} 1 \mathrm{C}-\mathrm{C} 6 \mathrm{C}-\mathrm{C} 5 \mathrm{C}$ & $-2.5(6)$ \\
\hline $\mathrm{C} 2 \mathrm{C}-\mathrm{C} 1 \mathrm{C}-\mathrm{C} 6 \mathrm{C}-\mathrm{C} 8 \mathrm{~B}$ & $178.2(3)$ \\
\hline $\mathrm{C} 2 \mathrm{~B}-\mathrm{C} 8 \mathrm{~B}-\mathrm{C} 6 \mathrm{C}-\mathrm{C} 5 \mathrm{C}$ & $-89.6(5)$ \\
\hline $\mathrm{C} 9 \mathrm{~B}-\mathrm{C} 8 \mathrm{~B}-\mathrm{C} 6 \mathrm{C}-\mathrm{C} 5 \mathrm{C}$ & $143.3(4)$ \\
\hline $\mathrm{C} 2 \mathrm{~B}-\mathrm{C} 8 \mathrm{~B}-\mathrm{C} 6 \mathrm{C}-\mathrm{C} 1 \mathrm{C}$ & $89.7(4)$ \\
\hline $\mathrm{C} 9 \mathrm{~B}-\mathrm{C} 8 \mathrm{~B}-\mathrm{C} 6 \mathrm{C}-\mathrm{C} 1 \mathrm{C}$ & $-37.4(5)$ \\
\hline $\mathrm{C} 3 \mathrm{C}-\mathrm{C} 2 \mathrm{C}-\mathrm{C} 8 \mathrm{C}-\mathrm{C} 6 \mathrm{D}$ & $88.4(4)$ \\
\hline $\mathrm{C} 1 \mathrm{C}-\mathrm{C} 2 \mathrm{C}-\mathrm{C} 8 \mathrm{C}-\mathrm{C} 6 \mathrm{D}$ & $-90.4(4)$ \\
\hline $\mathrm{C} 3 \mathrm{C}-\mathrm{C} 2 \mathrm{C}-\mathrm{C} 8 \mathrm{C}-\mathrm{C} 9 \mathrm{C}$ & $-143.8(4)$ \\
\hline $\mathrm{C} 1 \mathrm{C}-\mathrm{C} 2 \mathrm{C}-\mathrm{C} 8 \mathrm{C}-\mathrm{C} 9 \mathrm{C}$ & $37.5(5)$ \\
\hline $\mathrm{C} 6 \mathrm{D}-\mathrm{C} 8 \mathrm{C}-\mathrm{C} 9 \mathrm{C}-\mathrm{C} 10 \mathrm{C}$ & $-62.4(5)$ \\
\hline $\mathrm{C} 2 \mathrm{C}-\mathrm{C} 8 \mathrm{C}-\mathrm{C} 9 \mathrm{C}-\mathrm{C} 10 \mathrm{C}$ & $173.4(4)$ \\
\hline $\mathrm{C} 8 \mathrm{C}-\mathrm{C} 9 \mathrm{C}-\mathrm{C} 10 \mathrm{C}-\mathrm{C} 11 \mathrm{C}$ & $-176.4(4)$ \\
\hline
\end{tabular}




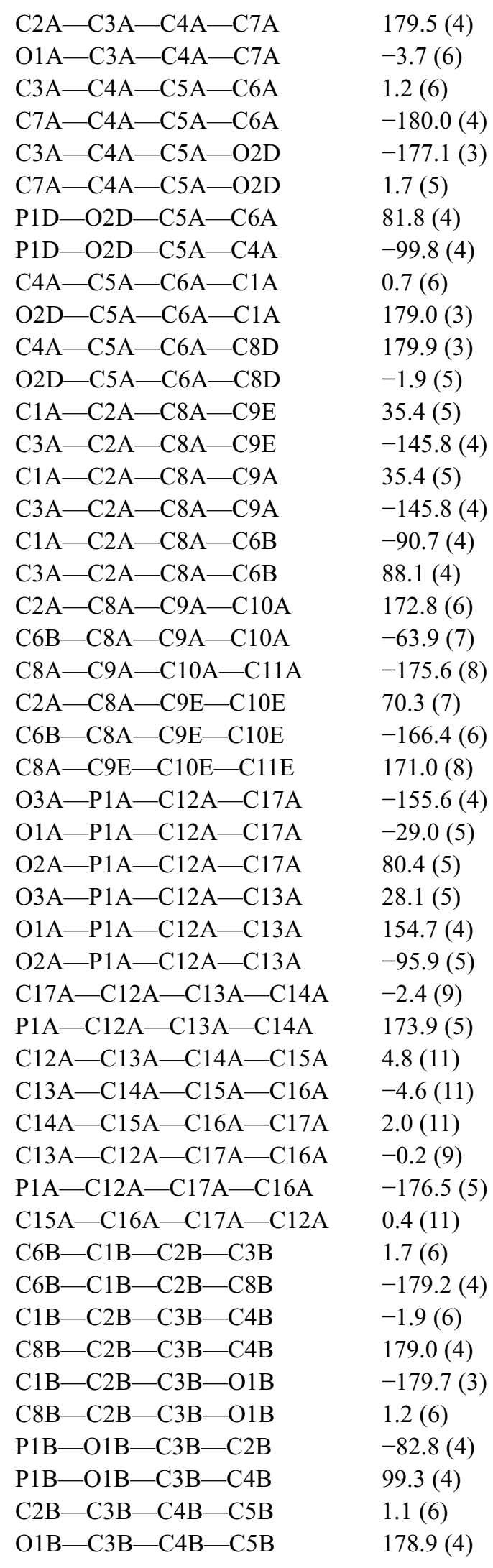

$179.5(4)$

$-3.7(6)$

$1.2(6)$

$-180.0(4)$

$-177.1(3)$

$1.7(5)$

$81.8(4)$

$-99.8(4)$

$0.7(6)$

$179.0(3)$

$179.9(3)$

$-1.9(5)$

$35.4(5)$

$-145.8(4)$

$35.4(5)$

$-145.8(4)$

$-90.7(4)$

$88.1(4)$

$172.8(6)$

$-63.9(7)$

$-175.6(8)$

$70.3(7)$

$-166.4(6)$

$171.0(8)$

-155.6 (4)

$-29.0(5)$

80.4 (5)

28.1 (5)

154.7 (4)

$-95.9(5)$

-2.4 (9)

$173.9(5)$

4.8 (11)

$-4.6(11)$

2.0 (11)

-0.2 (9)

$-176.5(5)$

0.4 (11)

1.7 (6)

$-179.2(4)$

$-1.9(6)$

179.0 (4)

-179.7 (3)

$1.2(6)$

$-82.8(4)$

99.3 (4)

1.1 (6)

$178.9(4)$

$\begin{array}{ll}\text { O3C-P1C-C12C-C13C } & 8.6(5) \\ \text { O1C-P1C-C12C-C13C } & 135.1(4) \\ \text { O2C-P1C-C12C-C13C } & -115.7(4) \\ \text { O3C-P1C-C12C-C17C } & -177.2(4) \\ \text { O1C-P1C-C12C-C17C } & -50.8(4) \\ \text { O2C-P1C-C12C-C17C } & 58.4(4) \\ \text { C17C-C12C-C13C-C14C } & -0.2(8) \\ \text { P1C-C12C-C13C-C14C } & 174.0(4) \\ \text { C12C-C13C-C14C-C15C } & 0.2(9) \\ \text { C13C-C14C-C15C-C16C } & 0.2(10) \\ \text { C14C-C15C-C16C-C17C } & -0.6(9) \\ \text { C13C-C12C-C17C-C16C } & -0.2(7) \\ \text { P1C-C12C-C17C-C16C } & -174.3(4) \\ \text { C15C-C16C-C17C-C12C } & 0.6(8) \\ \text { C6D-C1D-C2D-C3D } & 1.0(6) \\ \text { C6D-C1D-C2D-C8D } & 179.1(4) \\ \text { C1D-C2D-C3D-C4D } & -1.1(6) \\ \text { C8D-C2D-C3D-C4D } & -179.2(4) \\ \text { C1D-C2D-C3D-O1D } & -178.0(3) \\ \text { C8D-C2D-C3D-O1D } & 3.9(6) \\ \text { P1D-O1D-C3D-C4D } & 99.1(4) \\ \text { P1D-O1D-C3D-C2D } & -83.9(4) \\ \text { C2D-C3D-C4D-C5D } & 0.2(6) \\ \text { O1D-C3D-C4D-C5D } & 177.2(3) \\ \text { C2D-C3D-C4D-C7D } & -177.7(4) \\ \text { O1D-C3D-C4D-C7D } & -0.7(6) \\ \text { C3D-C4D-C5D-C6D } & 0.9(6) \\ \text { C7D-C4D-C5D-C6D } & 178.8(4) \\ \text { C3D-C4D-C5D-O2C } & -175.3(3) \\ \text { C7D-C4D-C5D-O2C } & 2.6(6) \\ \text { P1C-O2C-C5D-C6D } & 87.6(4) \\ \text { P1C-O2C-C5D-C4D } & -96.0(4) \\ \text { C4D-C5D-C6D-C1D } & -1.0(6) \\ \text { O2C-C5D-C6D-C1D } & 175.2(3) \\ \text { C4D-C5D-C6D-C8C } & 176.3(4) \\ \text { O2C-C5D-C6D-C8C } & -7.5(6) \\ \text { C2D-C1D-C6D-C5D } & 0.0(6) \\ \text { C2D-C1D-C6D-C8C } & -177.3(4) \\ \text { C9C-C8C-C6D-C5D } & 148.0(4) \\ \text { C2C-C8C-C6D-C5D } & -85.7(5) \\ \text { C9C-C8C-C6D-C1D } & -34.8(5) \\ \text { C2C-C8C-C6D-C1D } & 91.5(4) \\ \text { C1A-C6A-C8D-C2D } & 88.8(4) \\ \text { C5A-C6A-C8D-C2D } & -90.4(4) \\ \text { C1A-C6A-C8D-C9D } & -38.9(5) \\ \text { C5A-C6A-C8D-C9D } & 142.0(4) \\ \text { C3D-C2D-C8D-C6A } & 88.4(4) \\ \text { C1D-C2D-C8D-C6A } & -89.6(4) \\ & \end{array}$




$\begin{array}{ll}\mathrm{C} 2 \mathrm{~B}-\mathrm{C} 3 \mathrm{~B}-\mathrm{C} 4 \mathrm{~B}-\mathrm{C} 7 \mathrm{~B} & -178.7(4) \\ \mathrm{O} 1 \mathrm{~B}-\mathrm{C} 3 \mathrm{~B}-\mathrm{C} 4 \mathrm{~B}-\mathrm{C} 7 \mathrm{~B} & -0.9(6) \\ \mathrm{C} 3 \mathrm{~B}-\mathrm{C} 4 \mathrm{~B}-\mathrm{C} 5 \mathrm{~B}-\mathrm{C} 6 \mathrm{~B} & 0.1(6) \\ \mathrm{C} 7 \mathrm{~B}-\mathrm{C} 4 \mathrm{~B}-\mathrm{C} 5 \mathrm{~B}-\mathrm{C} 6 \mathrm{~B} & 179.9(4) \\ \mathrm{C} 3 \mathrm{~B}-\mathrm{C} 4 \mathrm{~B}-\mathrm{C} 5 \mathrm{~B}-\mathrm{O} 2 \mathrm{~A} & -177.8(3) \\ \mathrm{C} 7 \mathrm{~B}-\mathrm{C} 4 \mathrm{~B}-\mathrm{C} 5 \mathrm{~B}-\mathrm{O} 2 \mathrm{~A} & 2.0(6) \\ \mathrm{P} 1 \mathrm{~A}-\mathrm{O} 2 \mathrm{~A}-\mathrm{C} 5 \mathrm{~B}-\mathrm{C} 6 \mathrm{~B} & 85.2(4) \\ \mathrm{P} 1 \mathrm{~A}-\mathrm{O} 2 \mathrm{~A}-\mathrm{C} 5 \mathrm{~B}-\mathrm{C} 4 \mathrm{~B} & -96.8(4) \\ \mathrm{C} 4 \mathrm{~B}-\mathrm{C} 5 \mathrm{~B}-\mathrm{C} 6 \mathrm{~B}-\mathrm{C} 1 \mathrm{~B} & -0.3(6) \\ \mathrm{O} 2 \mathrm{~A}-\mathrm{C} 5 \mathrm{~B}-\mathrm{C} 6 \mathrm{~B}-\mathrm{C} 1 \mathrm{~B} & 177.5(3) \\ \mathrm{C} 4 \mathrm{~B}-\mathrm{C} 5 \mathrm{~B}-\mathrm{C} 6 \mathrm{~B}-\mathrm{C} 8 \mathrm{~A} & 177.5(4) \\ \mathrm{O} 2 \mathrm{~A}-\mathrm{C} 5 \mathrm{~B}-\mathrm{C} 6 \mathrm{~B}-\mathrm{C} 8 \mathrm{~A} & -4.7(6) \\ \mathrm{C} 2 \mathrm{~B}-\mathrm{C} 1 \mathrm{~B}-\mathrm{C} 6 \mathrm{~B}-\mathrm{C} 5 \mathrm{~B} & -0.6(6) \\ \mathrm{C} 2 \mathrm{~B}-\mathrm{C} 1 \mathrm{~B}-\mathrm{C} 6 \mathrm{~B}-\mathrm{C} 8 \mathrm{~A} & -178.4(4) \\ \mathrm{C} 9 \mathrm{E}-\mathrm{C} 8 \mathrm{~A}-\mathrm{C} 6 \mathrm{~B}-\mathrm{C} 5 \mathrm{~B} & 145.5(4) \\ \mathrm{C} 9 \mathrm{~A}-\mathrm{C} 8 \mathrm{~A}-\mathrm{C} 6 \mathrm{~B}-\mathrm{C} 5 \mathrm{~B} & 145.5(4) \\ \mathrm{C} 2 \mathrm{~A}-\mathrm{C} 8 \mathrm{~A}-\mathrm{C} 6 \mathrm{~B}-\mathrm{C} 5 \mathrm{~B} & -88.4(5) \\ \mathrm{C} 9 \mathrm{E}-\mathrm{C} 8 \mathrm{~A}-\mathrm{C} 6 \mathrm{~B}-\mathrm{C} 1 \mathrm{~B} & -36.9(6) \\ \mathrm{C} 9 \mathrm{~A}-\mathrm{C} 8 \mathrm{~A}-\mathrm{C} 6 \mathrm{~B}-\mathrm{C} 1 \mathrm{~B} & -36.9(6) \\ \mathrm{C} 2 \mathrm{~A}-\mathrm{C} 8 \mathrm{~A}-\mathrm{C} 6 \mathrm{~B}-\mathrm{C} 1 \mathrm{~B} & 89.2(5) \\ \mathrm{C} 3 \mathrm{~B}-\mathrm{C} 2 \mathrm{~B}-\mathrm{C} 8 \mathrm{~B}-\mathrm{C} 9 \mathrm{~B} & -144.5(4) \\ \mathrm{C} 1 \mathrm{~B}-\mathrm{C} 2 \mathrm{~B}-\mathrm{C} 8 \mathrm{~B}-\mathrm{C} 9 \mathrm{~B} & 36.4(5) \\ \mathrm{C} 3 \mathrm{~B}-\mathrm{C} 2 \mathrm{~B}-\mathrm{C} 8 \mathrm{~B}-\mathrm{C} 6 \mathrm{C} & 89.4(5) \\ \mathrm{C} 1 \mathrm{~B}-\mathrm{C} 2 \mathrm{~B}-\mathrm{C} 8 \mathrm{~B}-\mathrm{C} 6 \mathrm{C} & -89.7(5)\end{array}$

$\begin{array}{ll}\mathrm{C} 3 \mathrm{D}-\mathrm{C} 2 \mathrm{D}-\mathrm{C} 8 \mathrm{D}-\mathrm{C} 9 \mathrm{D} & -143.4(4) \\ \mathrm{C} 1 \mathrm{D}-\mathrm{C} 2 \mathrm{D}-\mathrm{C} 8 \mathrm{D}-\mathrm{C} 9 \mathrm{D} & 38.6(5) \\ \mathrm{C} 11 \mathrm{D}-\mathrm{C} 10 \mathrm{D}-\mathrm{C} 9 \mathrm{D}-\mathrm{C} 8 \mathrm{D} & -169.4(5) \\ \mathrm{C} 6 \mathrm{~A}-\mathrm{C} 8 \mathrm{D}-\mathrm{C} 9 \mathrm{D}-\mathrm{C} 10 \mathrm{D} & -57.6(5) \\ \mathrm{C} 2 \mathrm{D}-\mathrm{C} 8 \mathrm{D}-\mathrm{C} 9 \mathrm{D}-\mathrm{C} 10 \mathrm{D} & 176.8(4) \\ \mathrm{C} 5 \mathrm{~A}-\mathrm{C} 6 \mathrm{~A}-\mathrm{C} 1 \mathrm{~A}-\mathrm{C} 2 \mathrm{~A} & -2.4(6) \\ \mathrm{C} 8 \mathrm{D}-\mathrm{C} 6 \mathrm{~A}-\mathrm{C} 1 \mathrm{~A}-\mathrm{C} 2 \mathrm{~A} & 178.4(4) \\ \mathrm{C} 3 \mathrm{~A}-\mathrm{C} 2 \mathrm{~A}-\mathrm{C} 1 \mathrm{~A}-\mathrm{C} 6 \mathrm{~A} & 2.0(6) \\ \mathrm{C} 8 \mathrm{~A}-\mathrm{C} 2 \mathrm{~A}-\mathrm{C} 1 \mathrm{~A}-\mathrm{C} 6 \mathrm{~A} & -179.2(4) \\ \text { O3D-P1D-C12D-C17D } & -151.1(4) \\ \text { O1D-P1D-C12D-C17D } & -25.4(5) \\ \text { O2D-P1D-C12D-C17D } & 83.9(5) \\ \text { O3D-P1D-C12D-C13D } & 25.5(5) \\ \text { O1D-P1D-C12D-C13D } & 151.2(5) \\ \text { O2D-P1D-C12D-C13D } & -99.5(5) \\ \text { C17D-C12D-C13D-C14D } & -0.5(10) \\ \text { P1D-C12D-C13D-C14D } & -177.3(6) \\ \text { C12D-C13D-C14D-C15D } & -4.0(13) \\ \text { C13D-C14D-C15D-C16D } & 4.8(12) \\ \text { C14D-C15D-C16D-C17D } & -1.2(10) \\ \text { C13D-C12D-C17D-C16D } & 4.0(9) \\ \text { P1D-C12D-C17D-C16D } & -179.4(5) \\ \text { C15D-C16D-C17D-C12D } & -3.3(10) \\ & \end{array}$

Hydrogen-bond geometry $\left(A,{ }^{\circ}\right)$

$\mathrm{Cg} 1$ and $\mathrm{Cg} 2$ are the centroids of the rings $\mathrm{C} 1 B-\mathrm{C} 6 B$ and $\mathrm{C} 1 D-\mathrm{C} 6 D$, respectively.

\begin{tabular}{lllll}
\hline$D-\mathrm{H} \cdots A$ & $D-\mathrm{H}$ & $\mathrm{H} \cdots A$ & $D \cdots A$ & $D-\mathrm{H} \cdots A$ \\
\hline $\mathrm{N} 1-\mathrm{H} 1 A \cdots \mathrm{O} 3 A$ & 0.91 & 1.91 & $2.773(5)$ & 157 \\
$\mathrm{~N} 1-\mathrm{H} 1 B \cdots \mathrm{O} 3 B$ & 0.91 & 1.99 & $2.841(5)$ & 155 \\
$\mathrm{C} 2-\mathrm{H} 2 \cdots \mathrm{O} 3 D$ & 1.00 & 2.25 & $3.140(6)$ & 148 \\
$\mathrm{C} 3-\mathrm{H} 3 A \cdots \mathrm{O} 3 C$ & 0.98 & 2.49 & $3.351(7)$ & 147 \\
$\mathrm{C} 3-\mathrm{H} 3 B \cdots \mathrm{O} 1 S$ & 0.98 & 2.55 & $3.51(2)$ & 164 \\
$\mathrm{O} 2 S-\mathrm{H} 2 S \cdots \mathrm{C} 11$ & 0.84 & 2.28 & $3.105(5)$ & 169 \\
$\mathrm{C} 1 A-\mathrm{H} 1 A 1 \cdots \mathrm{C} 1$ & 0.95 & 2.91 & $3.847(4)$ & 170 \\
$\mathrm{C} 1 B-\mathrm{H} 1 B 1 \cdots \mathrm{Cl} 1$ & 0.95 & 2.93 & $3.870(5)$ & 170 \\
$\mathrm{C} 1 C-\mathrm{H} 1 C 1 \cdots \mathrm{Cl} 1$ & 0.95 & 2.95 & $3.888(5)$ & 170 \\
$\mathrm{C} 1 D-\mathrm{H} 1 D 1 \cdots \mathrm{Cl} 1$ & 0.95 & 2.85 & $3.782(5)$ & 168 \\
$\mathrm{C} 9 A-\mathrm{H} 9 A 1 \cdots \mathrm{Cl} 1$ & 0.99 & 2.76 & $3.738(5)$ & 172 \\
$\mathrm{C} 9 B-\mathrm{H} 9 B 2 \cdots \mathrm{C} 11$ & 0.99 & 2.88 & $3.870(4)$ & 175 \\
$\mathrm{C} 9 C-\mathrm{H} 9 C 1 \cdots \mathrm{C} 11$ & 0.99 & 2.71 & $3.701(5)$ & 175 \\
$\mathrm{C} 9 D-\mathrm{H} 9 D 1 \cdots \mathrm{Cl} 1$ & 0.99 & 2.85 & $3.838(5)$ & 178 \\
$\mathrm{C} 1-\mathrm{H} 1 D \cdots \mathrm{C} 1$ & 0.98 & 2.83 & $3.672(7)$ & 145 \\
$\mathrm{C} 17 D^{\mathrm{i}}-\mathrm{H} 17 D^{\mathrm{i}} \cdots \mathrm{O} 1$ & 0.95 & 2.56 & $3.204(6)$ & 125 \\
$\mathrm{C} 10-\mathrm{H} 10 \cdots \mathrm{O} 1 D^{\mathrm{i}}$ & 0.95 & 2.69 & $3.555(4)$ & 152
\end{tabular}




\begin{tabular}{lllll}
$\mathrm{C} 9-\mathrm{H} 9 \cdots \mathrm{Cg} 2^{\mathrm{i}}$ & 0.95 & 2.69 & $3.594(5)$ & 159 \\
$\mathrm{C} 14 B-\mathrm{H} 14 B^{\cdots} \cdots \mathrm{C} 11^{\mathrm{ii}}$ & 0.95 & 2.89 & $3.697(6)$ & 143 \\
\hline
\end{tabular}

Symmetry codes: (i) $-x+1, y+1 / 2,-z+3 / 2$; (ii) $-x, y+1 / 2,-z+3 / 2$. 